\title{
Phenomenological Implications of
}

\section{The Electroweak-scale Right-handed}

\section{Neutrino Model}

\author{
Vinh Van Hoang \\ Thai Binh, Viet Nam
}

M.Sc. Physics, Viet Nam National University,

Ha Noi, Viet Nam, 2007

B.Sc. Educational Physics, Ha Noi, Viet Nam, 2004

A Dissertation presented to

the Graduate Faculty of the University of Virginia

in Candidacy for the Degree of

Doctor of Phisolophy

Department of Physics

University of Virginia

May, 2016 


\begin{abstract}
In this thesis, we present the model of right-handed neutrinos at electroweak scale $\left(\mathrm{EW} \nu_{R}\right)$ and its extended version under two crucial tests by the electroweak precision measurements and the discovery of the $125 \mathrm{GeV}$ SM-like Higgs boson. The key feature of the model is the existence of non-sterile electroweak scale righthanded neutrinos together with scalar triplets. We show that the $\mathrm{EW} \nu_{R}$ model with the new particle content satisfies well the constraint of the electroweak precision measurements through the contribution to the oblique parameters, $S, T, U$. Moreover, the $125 \mathrm{GeV}$ SM-like Higgs discovery necessitates extending the minimal EW $\nu_{R}$ model by adding one more complex scalar doublet and imposing a new symmetry. We present two very distinct scenarios in which the $125 \mathrm{GeV}$ Higgs boson candidate of the model behaves like and unlike the SM one. In both cases, the signal strength of the $125 \mathrm{GeV}$ candidate satisfies the experimental results of the LHC. The phenomenology of the heavy spin-zero states is also taken into account.
\end{abstract}




\section{ACKNOWLEDGMENTS}

First and foremost, I want to express my deepest gratitude to my mentor Dr. Pham Quang Hung for teaching me most of what I know about theoretical high energy physics and about the way high energy theory is done. His unrestrained way of thinking about physics really inspires me to tackle the new challenge in research. This thesis cannot be written without his guidance, encouragement, and unconditioned support.

I also want to thank all of the faculties and staffs of the Department of Physics. I want to thank Dr. Nilanga Nilanage, Dr. Blaine Norum, Dr. Maxim Bychkov, for supporting my teaching career. I want to thank Dr. Bob Hirosky for such an inspiring computational physics course that I took in the first semester at the Department. I also want to thank Dr. Hank Thacker for his wonderful lectures in quantum field theories course. I am grateful for all members of my thesis committee for their understanding and support. My thanks also go to all of the staffs at the Department for all kind-hearted help during my graduate tenure.

The main part of this thesis cannot be done without many discussions with my graduate fellow and collaborator, Dr. Ajinkya Kamat. I sincerely thank all of my colleagues, Dr. Satya Nandi, Dr. Shreyashi Chakdar, Dr. Kirtiman Gosh, Trinh Le for interesting discussions. The years I have spent in Charlottesville have been some of the best years of my life. I want to thank the entire graduate fellows for 
making the Department such an enjoyable place to study and live in.

I want to thank Minh-Dung Tran Dang, my beloved wife, for love, understanding, and constant companionship. Finally, I want to thank my family for supporting my decision to study physics. 


\section{Contents}

List of Tables ............................... vii

List of Figures . . . . . . . . . . . . . . . . ix

1 The Standard Model of Electroweak Interactions 1

1.1 Weak Interactions before Gauge Theories . . . . . . . . . . . . . 1

1.1.1 Fermi Theory .................... 1

1.1.2 Unitarity Violation . . . . . . . . . . . . . . . 3

1.1.3 Intermediate Vector Boson Theory . . . . . . . . . . . 4

1.1.4 The rise of $S U(2)$ Group $\ldots \ldots \ldots \ldots$

1.2 Gauge Theories . . . . . . . . . . . . . . . . 9

1.2.1 Gauge transformations . . . . . . . . . . . . . 9

1.2.2 The Gauge Theories of Weak Interactions . . . . . . . . 11

1.3 Spontaneous Symmetry Breaking (SSB) and the Higgs Mechanism 14

1.3.1 The concept of Spontaneous Symmetry Breaking . . . . . 14

1.3.2 The Brout-Englert-Higgs Mechanism . . . . . . . . . 17

1.4 The Standard Model of Electroweak Interactions . . . . . . . . . . 21

1.4.1 The Gauge Group of the Standard Model . . . . . . . . . . 21

1.4.2 The Standard Model as a Gauge Theory . . . . . . . . . 23

1.4.3 Fermion Masses . . . . . . . . . . . . . . . 28

1.4 .4 Summary ........................ 29

2 Neutrino masses: Facts, Origins, And Mechanisms 31

2.1 Neutrino Oscillations . . . . . . . . . . . . . . . . . 32

2.1 .1 Mass \& Flavor Eigenstates . . . . . . . . . . . . . 32

2.1.2 Neutrino Oscillations in Vacuum . . . . . . . . . 33

2.1.3 Experimental Results . . . . . . . . . . . . . . . 35 
2.2 Neutrinos Mass . . . . . . . . . . . . . . . . 36

2.2 .1 Dirac mass . . . . . . . . . . . . . . 36

2.2.2 Majorana mass . . . . . . . . . . . . . . 37

2.3 The Seesaw Mechanism . . . . . . . . . . . . . . . . 37

2.3 .1 The Mechanism . . . . . . . . . . . . . . . 37

2.3.2 The Left-Right Symmetric Model . . . . . . . . . . . . 39

2.3.3 Summary of The Chapter . . . . . . . . . . . . . . 40

3 The Model of Right-Handed Neutrinos at ElectroWeak Scale 42

3.1 Motivations . . . . . . . . . . . . . . . . . . . . 42

3.2 The Model . . . . . . . . . . . . . . . . . . . . . . . . . 44

3.2.1 The Fermion Sector . . . . . . . . . . . . . . . . 44

3.2 .2 The Scalar Sector . . . . . . . . . . . . . . . . . 48

3.2.3 The SSB of the $\mathrm{EW} \nu_{R}$ Model . . . . . . . . . . . . 50

3.2.4 The Mass Spectrum of the Scalars . . . . . . . . . . . 51

3.3 Summary of The EW $\nu_{R}$ Model . . . . . . . . . . . . . . 53

4 Electroweak Precision Measurements $\quad 55$

4.1 Oblique Parameters . . . . . . . . . . . . . . . 55

4.2 The Oblique Parameters of the EW $\nu_{R}$ Model . . . . . . . . . . . 57

4.3 The Contribution of Mirror Fermions to $S, T \ldots \ldots$

4.4 The Contribution of the Scalars to $S, T \ldots \ldots$. . . . . . 61

4.5 Numerical Analysis . . . . . . . . . . . . . . . . . . . 63

4.5.1 The Parameter Space . . . . . . . . . . . . . . . 64

4.5.2 The Unconstrained $S, T \ldots \ldots \ldots$. . . . . . 65

4.5.3 The Constrained $S$ and $T \ldots \ldots \ldots$. . . . . . . 68

4.5.4 The Dependence of $\widetilde{T}_{S}$ and $\widetilde{S}_{S}$ On the Mass Splittings . . . 73

4.6 Summary of The Chapter . . . . . . . . . . . . . . 76 
5.1 The EW $\nu_{R}$ Model in the Light of 125 GeV Higgs Discovery . . . . 79

5.1 .1 The $125 \mathrm{GeV}$ Higgs Boson . . . . . . . . . . . 79

5.1.2 The $125 \mathrm{GeV}$ Higgs Boson Candidate of The Original EW $\nu_{R}$ Model ..................... 80

5.2 The Extension . . . . . . . . . . . . . . . . . . . . . . . . . . . 81

5.3 The Spontaneous Symmetry Breaking . . . . . . . . . . . . . . 82

5.4 The 125 GeV Higgs Boson Candidate . . . . . . . . . . . . . . . . 88

5.4.1 The Decay The 125 GeV Higgs Boson Candidate . . . . . 89

5.4.2 The Signal Strength of The $125 \mathrm{GeV}$ Higgs Boson Candidate 92

5.4 .3 The parameter space . . . . . . . . . . . . . . 94

5.5 The Dual Nature of the $125 \mathrm{GeV}$ SM-like Candidate of The EW $\nu_{R}$ Model ............................ 97

5.5.1 The SM-like $H_{1}^{0}$ is The Dominant Component of $\widetilde{H}$. . . . 100

5.5.2 The SM-like $H_{1}^{0}$ is The Sub-dominant Component of $\widetilde{H}$. . 103

5.6 The Other Heavy States . . . . . . . . . . . . . . . . . 106

5.6.1 The heavy CP-even states . . . . . . . . . . . 106

5.6 .2 The CP-odd Heavy States . . . . . . . . . . . . . . . . 108

5.7 Summary of The Chapter . . . . . . . . . . . . . . . . . . . 119

6 Conclusions $\quad 121$

A.1 The couplings in the Extended EW $\nu_{R}$ Model . . . . . . . . . . . . 123

A.2 Loop Functions . . . . . . . . . . . . . . . . . . . . . 130

A.3 Amplitude of $H_{3}^{0} \rightarrow W W / Z Z$. . . . . . . . . . . . . . . 133 


\section{LIST OF TABLES}

2.1 The parameters of neutrino oscillations. . . . . . . . . .

5.1 Allowed ranges of VEVs and parameters defined in Eq. (5.3.11). All values are given in $\mathrm{GeV} \ldots \ldots \ldots \ldots$

5.2 In the ggF production mode of the Higgs boson, partial widths and branching ratios for various channels in $\mathrm{SM}$ (for $m_{H_{S M}}=$ $125.7 \mathrm{GeV}$ ) with total width $=4.17 \mathrm{E}-3 \mathrm{GeV}$, and the $\mathrm{EW} \nu_{R}$ model for Dr. Jekyll Example 2 scenario: $a_{1,1 M}=-0.0025$, where $m_{\widetilde{H}}=125.7 \mathrm{GeV}$, total width $=4.45 \mathrm{E}-3 \mathrm{GeV}$ and $\widetilde{H} \sim H_{1}^{0}$. All the partial widths are given in $\mathrm{GeV} \ldots \ldots \ldots$. . . . . .

5.3 Partial width of $H \rightarrow g g$ as the measure of the production cross section, partial widths and branching ratios for various channels in $\mathrm{SM}$ (for $m_{H_{S M}}=125.6 \mathrm{GeV}$ and total width $4.15 \mathrm{E}-03 \mathrm{GeV}$ ), $\widetilde{H} \sim H_{1}^{0 \prime}\left(\right.$ with $m_{\widetilde{H}}=125.6 \mathrm{GeV}$ and total width $1.34 \mathrm{E}-03 \mathrm{GeV}$ ). All the partial widths are given in GeV . . . . . . . . . . . . . 105

1 Yukawa couplings of the scalars with SM quarks and mirror-quarks in the $E W \nu_{R}$ model. . . . . . . . . . . . . . . . . . . . . 123

2 Yukawa couplings of the scalars with SM quarks and mirror-quarks in the EW $\nu_{R}$ model. (con't) . . . . . . . . . . . . . . . . . 124

3 Triple couplings of the scalars with one gauge boson. Here we use common factor: $\imath g\left(p-p^{\prime}\right)^{\mu}$, where $p\left(p^{\prime}\right)$ is the incoming momentum of the scalars.

4 Triple couplings of the scalars with one gauge boson. Here we use common factor: $\imath g\left(p-p^{\prime}\right)^{\mu}$, where $p\left(p^{\prime}\right)$ is the incoming momentum of the scalars. 
5 Triple couplings of the scalars with two gauge bosons. Here we use common factor: $\imath g M_{W} g^{\mu \nu} \ldots \ldots \ldots \ldots \ldots$

6 Quartic couplings of the scalars with the gauge bosons. Common factor: $\imath g^{2} g^{\mu \nu} \ldots \ldots \ldots \ldots \ldots \ldots \ldots$

7 Quartic couplings of the scalars and gauge bosons. Common factor: $\imath g^{2} g^{\mu \nu} \ldots \ldots \ldots \ldots \ldots$ 


\section{List OF Figures}

1.1 Fermi 4-fermion interaction model to describe $\beta$-decay . . . . . . 2

$1.22^{\text {nd }}$ order in Fermi 4-fermion interaction theory . . . . . . . . 3

1.3 The $\nu_{\mu} e^{-} \rightarrow \mu^{-} \nu_{e}$ process with $W$ in exchange. . . . . . . . 5

1.4 Hypothesize $\nu_{e} \bar{\nu}_{e} \rightarrow W_{L}^{+} W_{L}^{-}$with exchanging $e \ldots \ldots 6$

1.5 The additional diagram. . . . . . . . . . . . . . . . 7

1.6 The self-interaction of $W \ldots \ldots \ldots \ldots \ldots$

$1.7 \mu^{2}<0 \ldots \ldots \ldots \ldots \ldots \ldots \ldots$

$1.8 \mu^{2}>0 \ldots \ldots \ldots \ldots \ldots$

2.1 Fermi 4-fermion interaction model to describe $\beta$-decay . . . . . . 32

4.1 The fermionic contributions to the self-energy 2-point function of gauge vector bosons . . . . . . . . . . . . . . . . 58

4.2 The contribution to the self-energy diagrams of the gauge bosons 61

4.3 The contribution of the mirror fermions. . . . . . . . . . . 65

4.4 The contribution of the mirror fermions is always above the $2-\sigma$ constraint. Here the EW precision measurements constraints are $\widetilde{S}=-0.02 \pm 0.14, \widetilde{T}=0.06 \pm 0.14[23] \ldots \ldots 66$

4.5 The contribution of the scalars. . . . . . . . . . . . 67

4.6 The contribution of the scalars with the EW precision measurement constraints. . . . . . . . . . . . . . . . . 68

4.7 The total $\widetilde{T}$ vs $\widetilde{S}$ with the 1 and $2 \sigma$ experimental contours. . . . 69

$4.8 \widetilde{T}_{S}$ vs $\widetilde{T}_{M F}$ under the 1 and $2 \sigma$ experimental constraints. . . . . 70

$4.9 \widetilde{S}_{S}$ vs $\widetilde{S}_{M F}$ under the 1 and $2 \sigma$ experimental constraints. . . . . . 71

$4.10 \widetilde{T}$ vs $\sin \theta_{H}$ under the 1 and $2 \sigma$ experimental constraints. . . . . 72

$4.11 \widetilde{S}$ vs $\sin \theta_{H}$ under the 1 and $2 \sigma$ experimental constraints. . . . . 73 
4.12 The dependence of $\widetilde{T}_{S}, \widetilde{S}_{S}$ on $X=1-\frac{m_{H_{3}^{+}}}{m_{H_{3}^{0}}}$ and $Y=1-\frac{m_{H_{5}^{+}}}{m_{H_{5}^{0}}}$ at $m_{H_{5}^{++}}=200 \mathrm{GeV} \ldots \ldots \ldots \ldots \ldots \ldots$

4.13 The dependence of $\widetilde{T}_{S}, \widetilde{S}_{S}$ on $X=1-\frac{m_{H_{3}^{+}}}{m_{H_{3}^{0}}}$ and $Y=1-\frac{m_{H_{5}^{+}}}{m_{H_{5}^{0}}}$ at $m_{H_{5}^{++}}=500 \mathrm{GeV} \ldots \ldots \ldots \ldots \ldots \ldots \ldots$

4.14 The dependence of $\widetilde{T}_{S}, \widetilde{S}_{S}$ on $X=1-\frac{m_{H_{3}^{+}}}{m_{H_{3}^{0}}}$ and $Y=1-\frac{m_{H_{5}^{+}}}{m_{H_{5}^{0}}}$ at $m_{H_{5}^{++}}=200 \mathrm{GeV} \ldots \ldots \ldots \ldots \ldots \ldots \ldots \ldots$

4.15 The dependence of $\widetilde{T}_{S}, \widetilde{S}_{S}$ on $X=1-\frac{m_{H_{3}^{+}}}{m_{H_{3}^{0}}}$ and $Y=1-\frac{m_{H_{5}^{+}}}{m_{H_{5}^{0}}}$ at $m_{H_{5}^{++}}=500 \mathrm{GeV}$ for $\widetilde{S}_{S}$ and $m_{H_{5}^{++}}=350 \mathrm{GeV}$ for $\widetilde{T}_{S} \ldots \ldots$

5.1 The measured signal strength of the $125 \mathrm{GeV}$ SM-like Higgs boson at the $\mathrm{LHC} \ldots \ldots \ldots \ldots \ldots \ldots$

5.2 Figure shows the predictions of $\mu\left(\widetilde{H} \rightarrow b \bar{b}, \tau \bar{\tau}, \gamma \gamma, W^{+} W^{-}, Z Z\right)$ in the EW $\nu_{R}$ model for Examples 1 and 2 in Dr. Jekyll and Examples 1, 2 and 3 in $M r$. Hyde scenarios, in comparison with corresponding best fit values by CMS $[30,31,32,33]$. . . . . .

5.3 Predicted signal strength of $\widetilde{H}^{\prime} \rightarrow W^{+} W^{-}$in 4 example scenarios (blue and purple squares). The results of the search for SM-like Higgs boson up to $600 \mathrm{GeV}$ with the $1 \sigma$ (green band) and $2 \sigma$ (yellow band) limits on the SM background (dotted curve) and CMS data (solid black curve) are also displayed.

5.4 The production cross section times branching ratio in $\gamma \gamma$ channel of $H_{3}^{0}$ and $H_{3 M}^{0} \cdot m_{H_{3}^{0}}=130-850 \mathrm{GeV}, m_{H_{3 M}^{0}}=177-870 \mathrm{GeV} \ldots .$.

5.5 The production cross section times branching ratio in $\gamma \gamma$ channel of $H_{3}^{0}$ and $H_{3 M}^{0} \cdot m_{H_{3}^{0}}=130-750 \mathrm{GeV}, m_{H_{3 M}^{0}}=182-870 \mathrm{GeV} \ldots .$.

5.6 The production cross section times branching ratio in $\tau \bar{\tau}$ channel of $H_{3}^{0}$ and $H_{3 M}^{0} \cdot m_{H_{3}^{0}}=130-440 \mathrm{GeV}, m_{H_{3 M}^{0}}=177-601 \mathrm{GeV} \ldots \ldots$ 
5.7 The production cross section times branching ratio in $\tau \bar{\tau}$ channel of $H_{3}^{0}$ and $H_{3 M}^{0} \cdot m_{H_{3}^{0}}=130-440 \mathrm{GeV}, m_{H_{3 M}^{0}}=182-618 \mathrm{GeV} \ldots \ldots 116$

5.8 Ratio of strength signal in $W W / Z Z$ channel of $H_{3}^{0}$ comparing to $H_{S M} 118$

1 Feynman diagram of $H_{3}^{0} \rightarrow W^{+} W^{-}$. We have three generations of mirror quarks and three generations of mirror leptons _ . . . . . 133

2 Feynman diagram of $H_{3}^{0} \rightarrow Z Z$. Here, $f=u_{1}^{M}, d_{1}^{M}, u_{2}^{M}, d_{2}^{M}, u_{3}^{M}, d_{3}^{M}$, and three charged mirror leptons $l^{M} \ldots \ldots \ldots \ldots$ 


\section{Chapter 1}

\section{The Standard Model of Electroweak Interactions}

In this chapter, we describe the unified theory of weak and electromagnetic interactions of the elementary particles which is often referred as the Standard Model of Electroweak interactions. It is a non-Abelian gauge theory in which the local phase invariance is spontaneously broken to ensure that weak interactions are a short range as indicated by experiments. We review the development of the Standard Model through different steps.

\subsection{Weak Interactions before Gauge Theories}

\subsubsection{Fermi Theory}

In analogy to Quantum Electrodynamics, Fermi proposed the 4-fermion theory to describe weak interactions in $\beta$-decay in 1934:

$$
\mathcal{L}_{F}=-\frac{G_{F}}{\sqrt{2}}\left(\bar{p}(x) \gamma_{\mu} n(x)\right)\left(\bar{e}(x) \gamma^{\mu} \nu(x)\right)+\text { h.c. }
$$

here $G_{F}$ is an empirical constant called the Fermi constant. In fact, setting $c=$ $\hbar=1$,

$$
G_{F} \approx 10^{-5} / m_{p}^{2}=1.1663787(6) \times 10^{-5}\left(G e V^{-2}\right)
$$


It turns out the process in Eq.(1.1.1) gives the neutron a very long lifetime $\tau \approx$ $886(s)$. In a comparison, the mean lifetime of pion is $(2.6033 \pm 0.0005) \times 10^{-8} s$. In principal, the lifetime depends inversely on the decay width $\tau \approx 1 / \Gamma$. It means the decay width of the $\beta$-decay is very small comparing with the decay width of pion. Consequently, the coupling in the $\beta$-decay is very small. In other words, $\beta$-decay is a weak interaction. The process of $n \rightarrow p+e^{-}+\bar{\nu}$ can be visualized by:

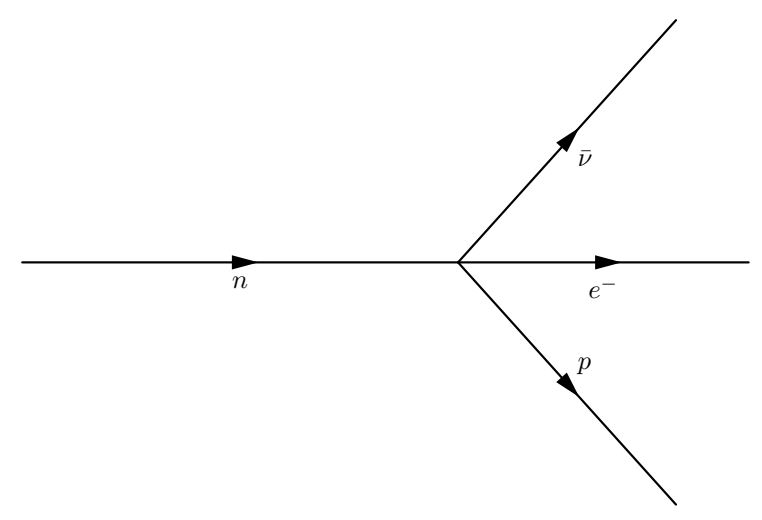

Figure 1.1. Fermi 4-fermion interaction model to describe $\beta$-decay

As we see, the Fermi model is purely phenomenological. It represents the interaction between two currents $J_{\mu}^{\text {weak }}=\bar{p}(x) \gamma_{\mu} n(x)$ and $J^{\text {weak, } \mu}=\bar{e}(x) \gamma^{\mu} \nu(x)$. The Lagrangian in Eq.(1.1.1) can be rewritten as:

$$
\mathcal{L}_{\text {weak }}=\frac{G_{F}}{\sqrt{2}} J_{\mu}^{\text {weak }} J^{\text {weak }, \mu}
$$

Unlike electromagnetic interactions, there exists charged currents in weak interactions, for example $J_{\mu}^{\text {weak }}=\bar{e}(x) \gamma_{\mu} \nu(x)$. Moreover, weak interactions are not always invariant under the Parity symmetry $P$. In the case of $J_{\mu}^{\text {weak }}=$ $\bar{p}(x) \gamma_{\mu} n(x), \bar{e}(x) \gamma_{\mu} \nu(x)$, then the weak interactions in these currents are invariant under $P$. However, $P$ is not conserved in the other processes such as the $\theta-\tau$ puzzle in Kaon system. The idea of $P$ non-conservation was proposed by Lee 
and Yang 1956 and later confirmed experimentally by Madam Wu et al 1957. The discovery of $P$ violation stimulated the research of weak interactions. Eventually, Feynman and Gell-man 1958; Marshak and Sudarshan 1958; and Sakurai 1958 completed the Vector minus Axial vector structure, in short V-A, of weak interactions. Finally, one had the form of weak currents:

$$
J_{\mu}^{\text {weak }}=\bar{e} \gamma_{\mu}\left(1-\gamma_{5}\right) \nu_{e}, \bar{\mu} \gamma_{\mu}\left(1-\gamma_{5}\right) \nu_{\mu}, \bar{p} \gamma_{\mu}\left(1-\gamma_{5}\right) n, \ldots
$$

In this form, it is clear that parity is violated

$$
\begin{aligned}
P \bar{\psi}(x) \gamma^{\mu} \gamma_{5} \psi(x) P^{-1} & =-\bar{\psi}(x) \gamma^{\mu} \gamma_{5} \psi(x) \\
P \bar{\psi}(x) \gamma^{\mu}\left(1-\gamma_{5}\right) \psi(x) P^{-1} & =\bar{\psi}(x) \gamma^{\mu}\left(1+\gamma_{5}\right) \psi(x)
\end{aligned}
$$

The V-A theory had been the center of achievements in studying weak interactions for many years. Until there was a sign of the bad behavior at high energy.

\subsubsection{Unitarity Violation}

In this part, we shall introduce the concept of intermediate vector boson theory based on unitarity argument. Let one consider a process such as process such as $\nu_{\mu} e^{-} \rightarrow \mu^{-} \nu_{e}$ at the higher order in perturbation theory, for example in the second order:

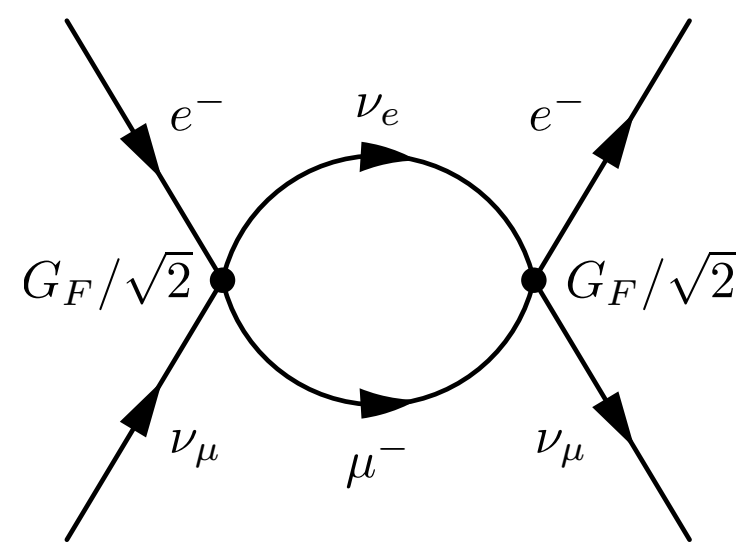

Figure 1.2. $2^{\text {nd }}$ order in Fermi 4-fermion interaction theory 
The cross-section of the process goes to infinity in the framework of Fermi theory. The origin of this problem lies in the violation of unitarity of the weak interactions described by the theory. In scattering theories, the $S$-matrix can be decomposed into the two parts:

$$
S=\mathbb{I}+i T
$$

Here $\mathbb{I}$ is the unit matrix representing the no-scattering part, while $T$ is the scattering matrix. Unitarity means that $S S^{\dagger}=S^{\dagger} S=1$. The cross section for the $\nu_{\mu} e^{-} \rightarrow \mu^{-} \nu_{e}$ process goes like $\sigma \approx G_{F}^{2} s$. Here $s=2 m_{e} E_{\nu}$; and $E_{\nu}$ is the energy of $\nu_{\mu}$ in the lab frame. In the partial wave expansion, the cross section is presented as $\sigma \approx \frac{\left|S_{J=1}\right|^{2}}{s}$. Unitarity requires $\left|S_{j=1}\right|^{2} \leq 1$ implying $\sigma \leq \frac{1}{s}$. The requirement of unitarity of $\nu_{\mu} e^{-} \rightarrow \mu^{-} \nu_{e}$ means:

$$
G_{F}^{2} s \leq \frac{1}{s} \rightleftharpoons \sqrt{s} \leq G_{F}^{-1 / 2} \approx 300 \mathrm{GeV}
$$

By this rough estimation, the theoretical cross-section of the $\nu_{\mu} e^{-} \rightarrow \mu^{-} \nu_{e}$ process would violate unitarity for energies above $300 \mathrm{GeV}$. In detail calculation, unitarity of weak interactions is violated at $E \approx 1 \mathrm{TeV}$.

\subsubsection{Intermediate Vector Boson Theory}

Quantum Electrodynamics, or QED, had been known for a "good" high energy behavior and being renormalizable. The coupling constant $e$ in QED is dimensionless. Whereas, in the Fermi theory of weak interactions, the coupling constant $G_{F}$ has the mass dimension of $[M]^{-2}$. To construct a fundamental theory for weak interactions, we could mimic the successful QED:

$$
\mathcal{L}^{Q E D}=e J_{\mu}^{e m} A^{\mu}
$$

here $A^{\mu}$ is the photon field which is responsible for carrying electrodynamic interactions. In the same way, one can express weak interactions in terms of weak 
current $J_{\mu}^{\text {weak }}$ and intermediate vector boson fields called $W^{\mu}$ :

$$
\mathcal{L}^{\text {weak }}=g J_{\mu}^{\text {weak }} W^{\mu}
$$

Weak interactions are carried via the propagator of $W^{\mu}$

$$
\frac{1}{q^{2}-m_{W}^{2}}
$$

Then the $\nu_{\mu} e^{-} \rightarrow \mu^{-} \nu_{e}$ process can be displayed in the Feynman diagram:

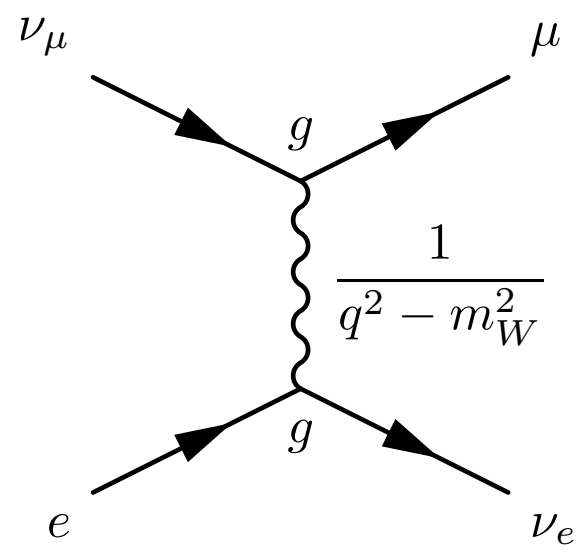

Figure 1.3. The $\nu_{\mu} e^{-} \rightarrow \mu^{-} \nu_{e}$ process with $W$ in exchange.

At low energy when $q^{2} \ll m_{W}^{2}$, then

$$
\frac{g^{2}}{q^{2}-m_{W}^{2}} \quad \longrightarrow \quad-\frac{g^{2}}{m_{W}^{2}} \equiv-\frac{G_{F}}{\sqrt{2}}
$$

Thus Fermi theory can be viewed as the low energy approximation.

Unfortunately, the theory with newly proposed vector bosons still has a bad high energy behavior. A massive spin-one field such as $W$ has 3 degrees of freedom: 2 transverse +1 longitudinal. Imagine that we are able to produce the process of $\nu_{e} \bar{\nu}_{e} \rightarrow W_{L}^{+} W_{L}^{-}:$ 


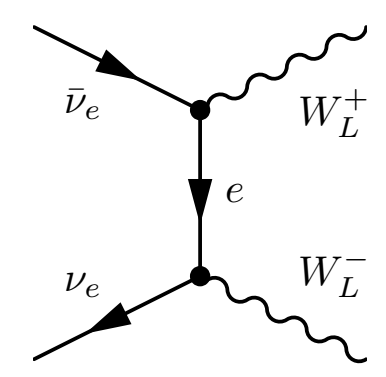

Figure 1.4. Hypothesize $\nu_{e} \bar{\nu}_{e} \rightarrow W_{L}^{+} W_{L}^{-}$with exchanging $e$.

The longitudinal polarization of $W^{ \pm}$has the form of $\epsilon_{\mu}^{(3)}=\frac{k_{\mu}}{M_{W}}+O\left(\frac{M_{W}}{k}\right)$. So the squared amplitude of the process has the term of

$$
\sum_{p o l} \epsilon_{\mu} \epsilon_{\nu}^{\star}=-g_{\mu \nu}+\frac{k_{\mu} k_{\nu}}{M_{W}^{2}}
$$

The part $k_{\mu} k_{\nu} / M_{W}^{2}$ causes the bad behavior at high energy. The cross section of this process is

$$
\sigma=\frac{G_{F}^{2} s}{12 \pi}
$$

$s$ is the energy of the process in the center of mass frame. On the other hand, in the partial wave expansion, the cross section can be expressed as

$$
\sigma=\frac{\pi}{E_{c m}^{2}} \sum_{J}(2 J+1)\left|S_{0,0,1 / 2,-1 / 2}^{J}\right|^{2}
$$

Here, $J$ is the angular momentum. Because only $J=1$ component contributes to the cross section, we have

$$
\left|S_{0,0,1 / 2,-1 / 2}^{1}\right|^{2}=\frac{G_{F}^{2} s^{2}}{36 \pi^{2}} \leq 1
$$

Or

$$
\sqrt{s} \leq \sqrt{\frac{12 \pi}{G_{F}}} \approx 1800 \mathrm{GeV} .
$$

Unitarity would be violated when the energy of the process in the center of mass frame $E_{c m}=\sqrt{s} / 2=900 \mathrm{GeV}$. We need a new development for the theory or tree-level weak interactions would be broken down at $1 \mathrm{TeV}$. 


\subsubsection{The rise of $S U(2)$ Group}

It turned out if the $W$ vector bosons have the symmetry of a Lie group, then the $\nu \bar{\nu} \rightarrow W_{L}^{+} W_{L}^{-}$process can be protected from the bad behavior at high energy by adding an extra diagram:

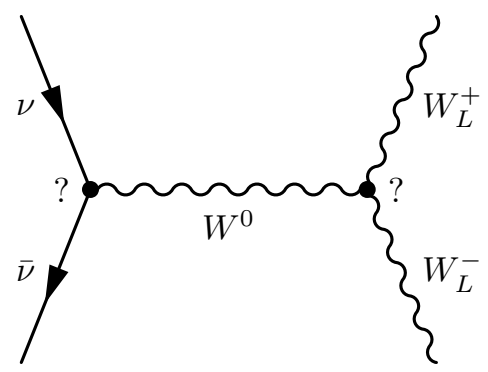

Figure 1.5. The additional diagram.

This diagram comes from the interaction of $W$ bosons themselves. Because $W$ bosons are spin 1 particles, they satisfy Bose symmetry. If we assume they have the same coupling constant, the amplitude of the self-interaction:

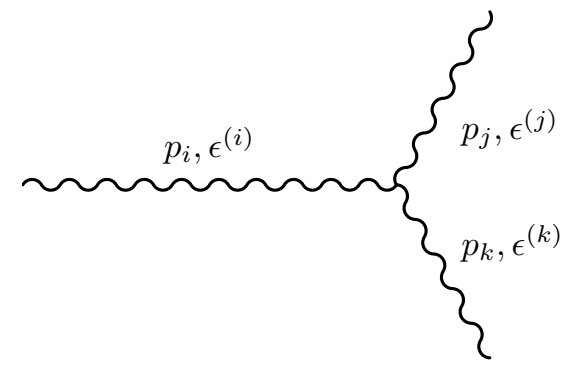

Figure 1.6. The self-interaction of $W$.

$$
\begin{aligned}
V & =i g\left(f^{i j, k}\left[\epsilon^{(i)} \cdot \epsilon^{(j)}\right]\left[\epsilon^{(k)}\left(p_{i}-p_{j}\right)\right]+f^{j k, i}\left[\epsilon^{(j)} \cdot \epsilon^{(k)}\right]\left[\epsilon^{(i)}\left(p_{j}-p_{k}\right)\right]\right. \\
& \left.+f^{k i, j}\left[\epsilon^{(k)} \cdot \epsilon^{(i)}\right]\left[\epsilon^{(j)}\left(p_{k}-p_{i}\right)\right]\right)
\end{aligned}
$$


With the contribution of the self-interaction process, the amplitude of the $\nu \bar{\nu} \rightarrow W_{L}^{+} W_{L}^{-}$process is

$$
\begin{aligned}
M^{\text {total }}= & -\frac{i}{M} g^{2} \bar{\nu}_{\alpha} \notin^{(i)}\left(\frac{1-\gamma_{5}}{2}\right)\left(T_{\alpha \rho}^{(i)} T_{\rho \beta}^{(j)}-T_{\alpha \rho}^{(j)} T_{\rho \beta}^{(i)}-i f^{k i, j} T_{\alpha \beta}^{(k)}\right) u_{\beta} \\
& +g^{2} \frac{\epsilon^{(i)} k^{(j)}}{M k^{(i)} k^{(j)}} \bar{\nu}_{\alpha} k^{(j)}\left(\frac{1-\gamma_{5}}{2}\right) T_{\alpha \beta}^{(k)} u_{\beta}\left(-f^{j k, i}+f^{i j, k}\right)
\end{aligned}
$$

As $k^{(i)} \rightarrow \infty$, the longitudinal $\epsilon^{(i)} \rightarrow \frac{k^{(i)}}{M}+O\left(\frac{M}{k}\right)$. As we see, $M^{\text {total }}$ depends on $k^{(i)}$, and increases with $k^{(i)}$. This is the bad behavior at high energy of the $\nu \bar{\nu} \rightarrow W_{L}^{+} W_{L}^{-}$process. Now, to prevent such a disaster, it is required $M^{\text {total }}=0$. This is equivalent to have two conditions:

$$
\begin{gathered}
f^{i j k}=f^{j k i}=f^{k i j} \\
{\left[T^{i}, T^{j}\right]=i f^{i j k} T^{k} .}
\end{gathered}
$$

In terms of group theory, $T^{i}$ s behave as the commutators of a Lie algebra. $T^{i}$ is the generator of the Lie algebra. $f^{i j k}$ is the structure constant. Three indices $i, j, k$ are corresponding to three generators of the Lie group. So it is natural to think of the group $S U(2)$. For this group, the generators can be expressed in terms of the Pauli matrices $\tau^{i}$.

$$
\tau^{1}=\left(\begin{array}{ll}
0 & 1 \\
1 & 0
\end{array}\right), \quad \tau^{2}=\left(\begin{array}{cc}
0 & -i \\
i & 0
\end{array}\right), \quad \tau^{3}=\left(\begin{array}{cc}
1 & 0 \\
0 & -1
\end{array}\right)
$$

They follow the algebra:

$$
\begin{aligned}
{\left[\tau^{i}, \tau^{j}\right] } & =2 i \epsilon^{i j k} \tau^{k} \\
\Leftrightarrow \quad\left[\frac{\tau^{i}}{2}, \frac{\tau^{j}}{2}\right] & =i \epsilon^{i j k} \frac{\tau^{k}}{2},
\end{aligned}
$$

here $\epsilon^{i j k}$ is the totally antisymmetric Levi-Civita symbol. So if $T^{i}=\frac{\tau^{i}}{2}$, then the Lie algebra in Eq.(1.1.19) can be realized with $f^{i j k}=\epsilon^{i j k}$.

So far, we briefly described the evolution of the theories of the weak interactions. The requirement of unitarity conservation lead ones to expand the theories 
from Fermi's four-fermion theory to the one with three vector bosons $W^{ \pm}, W^{0}$ which has the algebra of a group $S U(2)$ and a universal coupling $g$.

\subsection{Gauge Theories}

\subsubsection{Gauge transformations}

We can categorize a gauge transformation into two kinds, global and local, depending on the parameters of the transformation. Let us consider QED as an example of gauge theories.

\section{A global gauge transformation}

Considering the field $\Psi(x)$ and its conjugate under a phase transformation:

$$
\begin{aligned}
& \Psi(x) \rightarrow \Psi^{\prime}(x)=e^{-i \alpha} \Psi(x) \\
& \bar{\Psi}(x) \rightarrow \bar{\Psi}^{\prime}(x)=\bar{\Psi}(x) e^{i \alpha} .
\end{aligned}
$$

Here, $\alpha$ is a random phase factor which is independent of $x$. The probability term $\bar{\Psi}(x) \Psi(x)$ is invariant under the gauge transformation. Also, the kinetic term of $\Psi(x)$ is invariant:

$$
\begin{aligned}
\bar{\Psi}(x) \gamma^{\mu} \partial_{\mu} \Psi(x) & \rightarrow \bar{\Psi}(x) e^{i \alpha} \gamma^{\mu} \partial_{\mu} e^{-i \alpha} \Psi(x) \\
& =\bar{\Psi}(x) e^{i \alpha} e^{-i \alpha} \gamma^{\mu} \partial_{\mu} \Psi(x) \\
& =\bar{\Psi}(x) \gamma^{\mu} \partial_{\mu} \Psi(x) .
\end{aligned}
$$

It means that $\Psi(x)$ has a global $U(1)$ symmetry, a group with one parameter.

\section{A local gauge transformation}

When the phase in the transformation in Eq:(1.2.1) depends on $x$

$$
\begin{aligned}
& \Psi(x) \rightarrow \Psi^{\prime}(x)=e^{-i \alpha(x)} \Psi x \\
& \bar{\Psi}(x) \rightarrow \bar{\Psi}^{\prime}(x)=\bar{\Psi}(x) e^{i \alpha(x)} .
\end{aligned}
$$


We have a local gauge transformation. Then the probability term $\bar{\Psi}(x) \Psi(x)$ is still invariant under Eq.(1.2.3) transformation because

$$
\bar{\Psi}(x) \Psi(x) \rightarrow \bar{\Psi}^{\prime}(x) \Psi^{\prime}(x)=\bar{\Psi}(x) e^{i \alpha(x)} e^{-i \alpha(x)} \Psi(x)=\bar{\Psi}(x) \Psi(x)
$$

However the kinetic term is not:

$$
\begin{aligned}
\bar{\Psi}(x) \gamma^{\mu} \partial_{\mu} \Psi(x) & \rightarrow \bar{\Psi}(x) e^{i \alpha(x)} \gamma^{\mu} \partial_{\mu}\left(e^{-i \alpha(x)} \Psi(x)\right) \\
& =-i \bar{\Psi}(x) \gamma^{\mu} \Psi(x) \partial_{\mu} \alpha(x)+\bar{\Psi}(x) \gamma^{\mu} \partial_{\mu} \Psi(x)
\end{aligned}
$$

To preserve the invariance of this phase transformation, one needs to introduce a vector field $A_{\mu}(x)$ with a minimal coupling to the electron field $\Psi(x)$. Eventually, the field $A_{\mu}(x)$ can be viewed as the connection between different phase transformations at different points $x$. With the presence of the field $A_{\mu}(x)$, the kinetic term of $\Psi(x)$ becomes:

$$
\bar{\Psi}(x) i \gamma^{\mu}\left(\partial_{\mu}-i e A_{\mu}(x)\right) \Psi(x)
$$

Here,

$$
\partial_{\mu} \longrightarrow D_{\mu}=\partial_{\mu}-i e A_{\mu}(x)
$$

is called the covariant derivative. Under the local gauge transformation Eq.(1.2.3), the vector field $A_{\mu}(x)$ transforms as:

$$
A_{\mu}(x) \longrightarrow A_{\mu}^{\prime}(x)=A_{\mu}(x)-\frac{1}{e} \partial_{\mu} \alpha(x)
$$

Then the kinetic term in Eq.(1.2.6) transforms as:

$$
\begin{aligned}
\bar{\Psi}(x) i \gamma^{\mu}\left(\partial_{\mu}-i e A_{\mu}(x)\right) \Psi(x) \longrightarrow & -i \bar{\Psi}(x) \gamma^{\mu} \Psi(x) \partial_{\mu} \alpha(x)+\bar{\Psi}(x) \gamma^{\mu} \partial_{\mu} \Psi(x)- \\
& -i e \bar{\Psi}(x) \gamma^{\mu} A_{\mu}(x) \Psi(x)+i \bar{\Psi}(x) \gamma^{\mu} \Psi(x) \partial_{\mu} \alpha(x) \\
= & \bar{\Psi}(x) i \gamma^{\mu}\left(\partial_{\mu}-i e A_{\mu}(x)\right) \Psi(x)
\end{aligned}
$$


So, with the introduction of the vector field $A_{\mu}(x)$ under the transformation in Eq.(1.2.8), the kinetic term is invariant under the local gauge transformation Eq.(1.2.3) as well. Eventually, $A_{\mu}(x)$ is the gauge field of QED, so called the photon field, which carries electromagnetic interactions.

Consider the transformation of the field strength $F_{\mu \nu}$ and the mass term of the photon field under the gauge transformations Eq.(1.2.3) and Eq.(1.2.8).

$$
\begin{aligned}
F_{\mu \nu}=\partial_{\mu} A_{\nu}-\partial_{\nu} A_{\mu} & \longrightarrow \partial_{\mu} A_{\nu}-\partial_{\nu} A_{\mu}-\frac{1}{e} \partial_{\mu} \partial_{\nu} \alpha(x)+\frac{1}{e} \partial_{\nu} \partial_{\mu} \alpha(x) \\
& =\partial_{\mu} A_{\nu}-\partial_{\nu} A_{\mu}
\end{aligned}
$$

is invariant. Consequently, the kinetic term of the photon field $-\frac{1}{4} F_{\mu \nu} F^{\mu \nu}$ is also invariant. But the mass term $-\frac{1}{2} m_{\gamma}^{2} A_{\mu} A^{\mu}$ is not:

$$
-\frac{1}{2} m_{\gamma}^{2} A_{\mu} A^{\mu} \longrightarrow-\frac{1}{2} m_{\gamma}^{2}\left(A_{\mu} A^{\mu}-\frac{1}{2} \partial_{\mu} \alpha A^{\mu}-\frac{1}{e} A_{\mu} \partial^{\mu} \alpha+\frac{1}{e^{2}} \partial_{\mu} \alpha \partial^{\mu} \alpha\right) .
$$

It means that we cannot introduce by hand the mass term for the photon field without breaking gauge invariance. That is why the photon is naturally massless in QED.

\subsubsection{The Gauge Theories of Weak Interactions}

As discussed in section 1.1, unitarity requires us to introduce the vector field $\vec{W}_{\mu}$. The simplest model of weak interactions includes:

$$
\begin{array}{r}
\Psi_{L}=\left(\begin{array}{c}
\Psi_{1} \\
\Psi_{2}
\end{array}\right)_{L} \\
\vec{W}_{\mu}=\left(W^{1}, W^{2}, W^{3}\right) \\
W^{ \pm}=\frac{W^{1} \mp i W^{2}}{\sqrt{2}}
\end{array}
$$


Let us consider the model under a local $S U(2)_{L}$ phase transformation:

$$
\begin{gathered}
U(x)=e^{-i \vec{T} \cdot \vec{\alpha}(x)} \\
\Psi_{L} \longrightarrow U(x) \Psi_{L} \\
\bar{\Psi}_{L} \longrightarrow \bar{\Psi}_{L} U^{-1}(x) .
\end{gathered}
$$

Here, $\vec{\alpha}(x)=\left(\alpha_{1}, \alpha_{2}, \alpha_{3}\right)$ are three parameters of the gauge transformation corresponding to three generators $\vec{T}=\left(T^{1}, T^{2}, T^{3}\right)=\frac{\vec{\tau}}{2}$ of the group $S U(2)_{L} . \quad \vec{\tau}$ here are Pauli matrices. The kinetic term $\bar{\Psi}_{L} i \gamma_{\mu} \partial^{\mu} \Psi_{L}$ is not invariant under this gauge transformation:

$$
\begin{aligned}
\bar{\Psi}_{L} i \gamma_{\mu} \partial^{\mu} \Psi_{L} & \longrightarrow \bar{\Psi}_{L} U^{-1}(x) i \gamma_{\mu} \partial^{\mu}\left(U(x) \Psi_{L}\right) \\
& =\bar{\Psi}_{L} i \gamma_{\mu} \partial^{\mu} \Psi_{L}+\bar{\Psi}_{L} i \gamma_{\mu}\left[U^{-1} \partial^{\mu} U\right] \Psi_{L}
\end{aligned}
$$

Introducing the covariant derivative:

$$
D_{\mu}=\partial_{\mu}-i g \vec{T} \cdot \vec{W}_{\mu}
$$

Under the phase transformation in Eq.(1.2.13):

$$
\vec{T} \cdot \vec{W}_{\mu} \longrightarrow \vec{T} \cdot \vec{W}_{\mu}^{\prime}=U \vec{T} \cdot \vec{W}_{\mu} U^{-1}-\frac{i}{g}\left(\partial_{\mu} U\right) U^{-1}
$$

Then transformation of the kinetic terms of the fermion field and the gauge fields are invariant under the gauge transformation in Eqs.1.2.13, 1.2.15:

- For the kinetic term of the fermion field

$$
\begin{aligned}
\bar{\Psi}_{L} i \gamma_{\mu} D^{\mu} \Psi_{L} & \longrightarrow \bar{\Psi}_{L} i \gamma_{\mu} \partial^{\mu} \Psi_{L}+\bar{\Psi}_{L} i \gamma_{\mu}\left[U^{-1} \partial^{\mu} U\right] \Psi_{L}+ \\
& +g \bar{\Psi}_{L} U^{-1} \gamma_{\mu}\left(U \vec{T} \cdot \vec{W}_{\mu} U^{-1}-\frac{i}{g}\left(\partial_{\mu} U\right) U^{-1}\right) U \Psi_{L} \\
& =\bar{\Psi}_{L} i \gamma_{\mu} D^{\mu} \Psi_{L}
\end{aligned}
$$


- For the kinetic term of the gauge boson fields

$$
-\frac{1}{4} G_{\mu \nu}^{i} G^{i \mu \nu}=-\frac{1}{2} \operatorname{Tr}\left[\vec{T} \cdot \vec{G}_{\mu \nu} \vec{T} \cdot \vec{G}^{\mu \nu}\right]
$$

While under the gauge transformation in Eqs. 1.2.13, 1.2.15,

$$
\begin{aligned}
\operatorname{Tr}\left[\vec{T} \cdot \vec{G}_{\mu \nu} \vec{T} \cdot \vec{G}^{\mu \nu}\right] \longrightarrow \operatorname{Tr}\left[\vec{T} \cdot \vec{G}_{\mu \nu}^{\prime} \vec{T} \cdot \vec{G}^{\mu \nu \prime}\right] & =\operatorname{Tr}\left[U \vec{T} \cdot \vec{G}_{\mu \nu} U^{-1} U \vec{T} \cdot \vec{G}^{\mu \nu} U^{-1}\right] \\
& =\operatorname{Tr}\left[\vec{T} \cdot \vec{G}_{\mu \nu} \vec{T} \cdot \vec{G}^{\mu \nu}\right]
\end{aligned}
$$

However, the mass term of the vector boson is not invariant under the gauge transformation in Eqs. 1.2.13, 1.2.15:

$$
\begin{aligned}
\vec{W}_{\mu} \cdot \vec{W}^{\mu} \longrightarrow \vec{W}_{\mu}^{\prime} \cdot \vec{W}^{\mu \prime}= & \left(W_{\mu}^{i}+\epsilon^{i j k} \alpha^{j} W_{\mu}^{k}-\frac{i}{g} \partial_{\mu} \alpha^{i}\right) \\
& \left(W^{\mu i}+\epsilon^{i j k} \alpha^{j} W^{\mu k}-\frac{i}{g} \partial^{\mu} \alpha^{i}\right) \\
\neq & \vec{W}_{\mu} \cdot \vec{W}^{\mu}
\end{aligned}
$$

So $W$ remains massless in this theory, which is in contradiction with the shortrange nature of weak interactions implied by experiment. On the other hand, quarks and leptons must have masses. The fermion mass term is:

$$
\begin{aligned}
m_{f} \bar{\Psi} \Psi & =m_{f}\left(\bar{\Psi}\left(\frac{1-\gamma_{5}}{2}\right) \Psi+\bar{\Psi}\left(\frac{1+\gamma_{5}}{2}\right) \Psi\right) \\
& =m_{f} \bar{\Psi}_{R} \Psi_{L}+m_{f} \bar{\Psi}_{L} \Psi_{R}
\end{aligned}
$$

Due to the absence of the $\mathrm{V}+\mathrm{A}$ current, right-handed fermions are singlets under the $S U(2)_{L}$. It means that under transformation in Eq.(1.2.3), $\Psi_{R} \longrightarrow \Psi_{R}$. So

$$
\begin{aligned}
& \bar{\Psi}_{L} \Psi_{R} \longrightarrow \bar{\Psi}_{L} U^{-1} \Psi_{R} \\
& \bar{\Psi}_{R} \Psi_{L} \longrightarrow \bar{\Psi}_{R} U \Psi_{L}
\end{aligned}
$$

Hence, the mass term of fermions is not invariant. Again, new development is a must. 


\subsection{Spontaneous Symmetry Breaking (SSB) and the Higgs Mechanism}

So far, unitary principle and gauge invariance principle have led us from the 4-fermion theory of Fermi to the intermediate vector boson theory. However, the mass terms of vector bosons and fermions would break gauge invariance explicitly. The Goldstone theorem about spontaneous symmetry breaking (SSB) and the Higgs Mechanism are the solutions to this dilemma. We devote this section to present these two pillars of modern particle physics.

\subsubsection{The concept of Spontaneous Symmetry Breaking}

The SSB happens in a theory when the Lagrangian is invariant under the symmetry but the ground state is not. The spontaneous breakdown of a continuous global symmetry implies the existence of massless spin-zero particles. The phenomenon of spontaneous symmetry breaking was first introduced in the GinzburgLandau theory (1950), but the study of the connection between the SSB and the massless spinless particles was investigated by Nambu (1960), Nambu and JonaLasinio (1961), and other authors afterward[1]. Such scalar particles are referred to as Nambu-Goldstone bosons. We will study a toy model as an illustrative example of the spontaneous breaking of a global symmetry.

Let us consider a 2-component spin-0 field $\Phi$ which transforms like a vector under the rotation group $O(2)$.

$$
\vec{\Phi}=\left(\begin{array}{l}
\sigma \\
\pi
\end{array}\right)
$$

Under the rotation of $O(2)$,

$$
\vec{\Phi} \longrightarrow \vec{\Phi}^{\prime}=\left(\begin{array}{l}
\sigma^{\prime} \\
\pi^{\prime}
\end{array}\right)=\left(\begin{array}{cc}
\cos \theta & \sin \theta \\
-\sin \theta & \cos \theta
\end{array}\right)\left(\begin{array}{l}
\sigma \\
\pi
\end{array}\right)
$$


The kinetic term has a form of:

$$
\begin{aligned}
\mathcal{L}_{\text {Kin }} & =\frac{1}{2} \partial_{\mu} \vec{\Phi} \cdot \partial^{\mu} \vec{\Phi} \\
& =\frac{1}{2}\left(\partial_{\mu} \sigma\right)^{2}+\frac{1}{2}\left(\partial_{\mu} \pi\right)^{2}
\end{aligned}
$$

The potential is

$$
\begin{aligned}
V(\vec{\Phi} \cdot \vec{\Phi}) & =-\frac{1}{2} \mu^{2} \vec{\Phi} \cdot \vec{\Phi}+\frac{\lambda}{4}(\vec{\Phi} \cdot \vec{\Phi})^{2} \\
& =-\frac{1}{2} \mu^{2}\left(\sigma^{2}+\pi^{2}\right)+\frac{\lambda}{4}\left(\sigma^{2}+\pi^{2}\right)^{2}
\end{aligned}
$$

Here, $\lambda>0$, and $\mu^{2}$ can be negative or positive. The shape of the potential and then the nature of the ground states depend on the $\mu^{2}$.

- For $\mu^{2}<0$, the potential has a parabolic shape

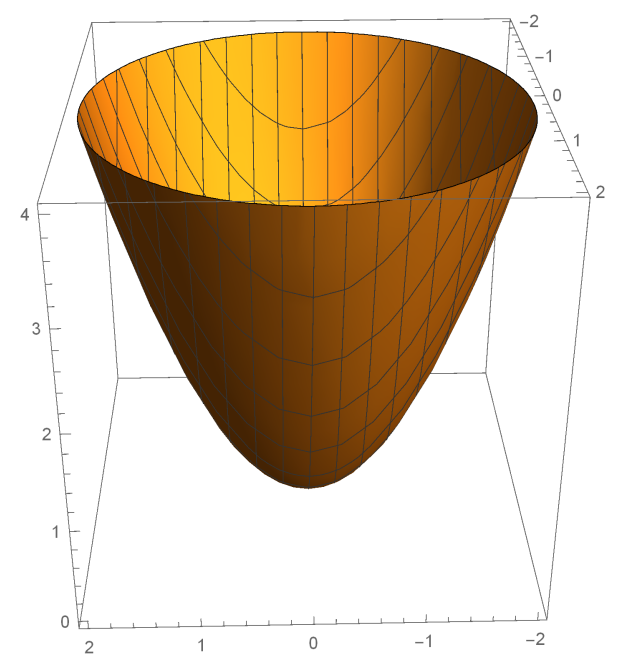

Figure 1.7. $\mu^{2}<0$

In this case, there is no spontaneous symmetry breaking.

- For $\mu^{2}>0$, the potential has a famous "Mexican hat" shape: 


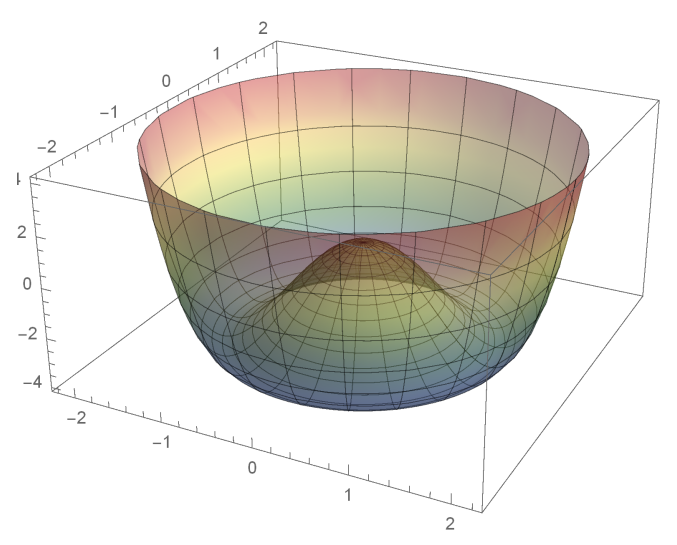

Figure 1.8. $\mu^{2}>0$

The Lagrangian is

$$
\begin{aligned}
\mathcal{L} & =\mathcal{L}_{\text {Kin }}-V(\vec{\Phi} \cdot \vec{\Phi}) \\
& =\frac{1}{2}\left(\partial_{\mu} \sigma\right)^{2}+\frac{1}{2}\left(\partial_{\mu} \pi\right)^{2}+\frac{1}{2} \mu^{2}\left(\sigma^{2}+\pi^{2}\right)-\frac{\lambda}{4}\left(\sigma^{2}+\pi^{2}\right)^{2}
\end{aligned}
$$

The ground state is found through the minimization conditions:

$$
\begin{aligned}
& \left\{\begin{array}{l}
\frac{\partial V}{\partial \sigma}=\sigma\left(-\mu^{2}+\lambda\left(\sigma^{2}+\pi^{2}\right)\right)=0 \\
\frac{\partial V}{\partial \pi}=\pi\left(-\mu^{2}+\lambda\left(\sigma^{2}+\pi^{2}\right)\right)=0
\end{array}\right. \\
& \Rightarrow \sigma_{0}^{2}+\pi_{0}^{2}=v^{2}=\frac{\mu^{2}}{\lambda} \Leftrightarrow v=\sqrt{\frac{\mu^{2}}{\lambda}}
\end{aligned}
$$

We have a freedom to choose $\left(\sigma_{0}, \pi_{o}\right)$ to satisfy the condition in Eq.(1.3.6). A particular solution is when

$$
\sigma_{0}=<0|\sigma| 0>=v, \quad \pi_{0}=<0|\pi| 0>=0
$$

We can make a shift:

$$
\sigma^{\prime}=\sigma-v \quad \Rightarrow<0\left|\sigma^{\prime}\right| 0>=<0|\sigma| 0>-v=0
$$


The Lagrangian in Eq. (1.3.5) becomes:

$$
\begin{aligned}
\mathcal{L} & =\frac{1}{2}\left(\partial_{\mu} \sigma^{\prime}\right)^{2}-\frac{1}{2}\left(2 \mu^{2}\right) \sigma^{\prime 2}-\lambda v \sigma^{\prime}\left(\sigma^{\prime 2}+\pi^{2}\right) \\
& -\frac{\lambda}{4}\left(\sigma^{\prime 2}+\pi^{2}\right)^{2}+\frac{1}{4} \frac{\mu^{2}}{\lambda}
\end{aligned}
$$

Note that $V\left(\sigma^{\prime}=\pi=0\right)=\frac{1}{4} \frac{\mu^{2}}{\lambda}$ which can be shifted away as we ignore gravity. There is no mass term for $\pi$ which is the massless Nambu-Goldstone boson of the model. From the quadratic term of $\sigma^{\prime}$, we can see $m_{\sigma^{\prime}}^{2}=2 \mu^{2} \Rightarrow m_{\sigma^{\prime}}=\sqrt{2} \mu$

\subsubsection{The Brout-Englert-Higgs Mechanism}

The main idea of the Brout-Englert-Higgs mechanism is that the massless gauge bosons becomes massive ones by absorbing the Nambu-Goldstone bosons appearing in the SSB of a local group [2]. In this mechanism, we introduce a new complex scalar field with non-zero vacuum expectation, so-called the Higgs field. A complex scalar has two degrees of freedom, real and imaginary. $U(1)$ is the group of the complex phase transformation. Therefore, this group should be the gauge group of $\Phi$. In group theory, $O(2) \sim U(1)$. We then apply the same procedure in the last part with the toy model. We can parameterize $\Phi$ in terms of two real fields $\Phi_{1}, \Phi_{2}$ :

$$
\begin{aligned}
\Phi & =\frac{1}{\sqrt{2}}\left(\Phi_{1}+i \Phi_{2}\right) \\
\Phi^{\dagger} & =\frac{1}{\sqrt{2}}\left(\Phi_{1}-i \Phi_{2}\right)
\end{aligned}
$$

Under the local gauge transformation $e^{-i \alpha(x)}$ :

$$
\begin{gathered}
\Phi(x) \longrightarrow \Phi^{\prime}(x)=e^{-i \alpha(x)} \Phi(x) \\
\partial_{\mu} \longrightarrow D_{\mu}=\partial_{\mu}-i g A_{\mu} \\
A_{\mu} \longrightarrow A_{\mu}^{\prime}=A_{\mu}-\frac{i}{g} \partial_{\mu} \alpha(x)
\end{gathered}
$$


The Lagrangian can be written as

$$
\begin{aligned}
\mathcal{L}_{K i n} & =\left(D_{\mu} \Phi\right)^{\dagger}\left(D^{\mu} \Phi\right)-\frac{1}{4} F_{\mu \nu} F^{\mu \nu} \\
V(\Phi) & =-\frac{1}{2} \mu^{2} \Phi^{\dagger} \Phi+\frac{\lambda}{4}\left(\Phi^{\dagger} \Phi\right)^{2}
\end{aligned}
$$

When $\mu^{2}>0$, the potential has the same as in Figure 1.8. At the minimum,

$$
\begin{gathered}
|\Phi|^{2}=\frac{1}{2}\left(\Phi_{1}^{2}+\Phi_{2}^{2}\right)=\frac{v^{2}}{2} \\
\Rightarrow \Phi_{1}^{2}+\Phi_{2}^{2}=v^{2}=\frac{\mu^{2}}{\lambda} .
\end{gathered}
$$

We can redefine $\Phi$ in a polar coordinate system:

$$
\begin{aligned}
\Phi(x) & =\frac{1}{\sqrt{2}}(v+\eta(x)) e^{i \xi(x)} / v \\
& \approx \frac{1}{\sqrt{2}}(v+\eta(x)+i \xi(x)+\cdots) .
\end{aligned}
$$

Thus the VEVs of $\left\langle\eta>_{o}=<\xi>_{o}=0\right.$. For a small oscillation, we can have a parameterization such as

$$
\left\{\begin{array}{l}
\Phi_{1}(x)=v+\eta(x) \\
\Phi_{2}(x)=\xi(x)
\end{array}\right.
$$

Under the gauge transformation:

$$
\begin{gathered}
\Phi(x) \longrightarrow \Phi^{\prime}(x)=e^{-i \xi(x) / v} \Phi(x)=\frac{1}{\sqrt{2}}(v+\eta(x)) \\
A_{\mu} \longrightarrow B_{\mu}=A_{\mu}-\frac{1}{g v} \partial_{\mu} \xi(x) .
\end{gathered}
$$

Then

$$
\begin{aligned}
\left(\partial_{\mu}-i g A_{\mu}\right) \Phi(x) & \longrightarrow\left(\partial_{\mu}-i g B_{\mu}\right) \Phi^{\prime}(x)=\frac{1}{\sqrt{2}}\left(\partial_{\mu} \eta(x)-i g B_{\mu}(v+\eta(x))\right) \\
\left|D_{\mu} \Phi(x)\right|^{2} & \longrightarrow \frac{1}{2}\left|\partial_{\mu} \eta(x)-i g B_{\mu}(v+\eta(x))\right|^{2} \\
& =\frac{1}{2}\left(\partial_{\mu} \eta(x)\right)^{2}+\frac{1}{2} g^{2} v^{2} B_{\mu} B^{\mu}+g^{2} v \eta(x) B_{\mu} B^{\mu}+\frac{1}{2} g^{2} \eta^{2}(x) B_{\mu} B^{\mu} .
\end{aligned}
$$


The Lagrangian becomes:

$$
\begin{aligned}
\mathcal{L} \longrightarrow \mathcal{L}^{\prime}= & \frac{1}{2}\left(\partial_{\mu} \eta\right)^{2}+\frac{\mu^{2}}{2}(v+\eta)^{2}-\frac{\lambda}{4}(v+\eta)^{4} \\
& +\frac{1}{2} g^{2} v^{2} B_{\mu} B^{\mu}+g^{2} v \eta B_{\mu} B^{\mu}+\frac{1}{2} g^{2} \eta^{2} B_{\mu} B^{\mu} \\
& -\frac{1}{4}\left(\partial_{\mu} B_{\nu}-\partial_{\nu} B_{\mu}\right)^{2}
\end{aligned}
$$

Using the minimization condition $v^{2}=\mu^{2} / \lambda$, we have

$$
\left\{\begin{array}{l}
\frac{\mu^{2}}{2}-\frac{\lambda}{4} 6 v^{2}=-\frac{1}{2} 2 \mu^{2} \\
\mu^{2} v-\frac{\lambda}{4} 4 v^{3}=0
\end{array}\right.
$$

We can simplify the Lagrangian:

$$
\begin{aligned}
\mathcal{L}^{\prime}= & \frac{1}{2}\left(\partial_{\mu} \eta\right)^{2}-\frac{1}{2} 2 \mu^{2} \eta^{2}-\lambda v \eta^{3}+\frac{1}{2} g^{2} v^{2} B_{\mu} B^{\mu}+\left(g^{2} v \eta+\frac{1}{2} g^{2} \eta^{2}\right) B_{\mu} B^{\mu} \\
& -\frac{1}{4}\left(\partial_{\mu} B_{\nu}-\partial_{\nu} B_{\mu}\right)^{2}
\end{aligned}
$$

$\xi$ disappears in the Lagrangian. Eventually, it is absorbed as the longitudinal component of $B_{\mu}$. From the Lagrangian, $m_{\eta}=\sqrt{2} \mu$, and $m_{B}=g v$. So we have one massive scalar $\eta$, and one massive vector $B_{\mu}$.

In the next step, we will apply the Higgs mechanism to weak interactions. Let us introduce a complex scalar doublet:

$$
\Phi=\left(\begin{array}{c}
\Phi_{1} \\
\Phi_{2}
\end{array}\right)
$$

here $\Phi_{1}, \Phi_{2}$ are complex scalar fields. So there are four degrees of freedom. The potential has the form of:

$$
V(\Phi)=-\frac{\mu^{2}}{2}\left(\Phi^{\dagger} \Phi\right)+\frac{\lambda}{4}\left(\Phi^{\dagger} \Phi\right)^{2}
$$

For weak interactions,

$$
\begin{aligned}
D_{\mu} \Phi & =\left(\partial_{\mu}-i g \frac{\vec{\tau}}{2} \vec{W}_{\mu}\right) \Phi \\
G_{\mu \nu}^{i} & =\partial_{\mu} W_{\nu}^{i}-\partial_{\nu} W_{\mu}^{i}+g \epsilon^{i j k} W_{\mu}^{j} W_{\nu}^{k}
\end{aligned}
$$


Choose a vacuum for the scalar:

$$
<\Phi>=\frac{1}{\sqrt{2}}\left(\begin{array}{l}
0 \\
v
\end{array}\right)
$$

Then make a shift:

$$
\Phi^{\prime}=\Phi-<\Phi>\Rightarrow<\Phi^{\prime}>=0
$$

Apply the gauge transformation as in Eq. (1.3.14). As seen in the last part, in a small oscillation, the shift in Eq. (1.3.23) is equivalent to

$$
\Phi \longrightarrow \Phi^{\prime}=e^{-i \frac{\vec{\tau} \cdot \vec{\xi}}{v}} \Phi=\left(\begin{array}{c}
0 \\
\frac{v+\eta}{\sqrt{2}}
\end{array}\right)
$$

Under this gauge transformation $U=e^{-i \frac{\vec{\tau} \cdot \vec{\xi}}{v}}$ :

$$
\begin{gathered}
\frac{\vec{\tau}}{2} \cdot \vec{A}_{\mu} \longrightarrow \frac{\vec{\tau}}{2} \cdot \vec{B}_{\mu}=U \frac{\vec{\tau}}{2} \cdot \vec{A}_{\mu} U^{-1}-\frac{i}{g}\left[\partial_{\mu} U\right] U^{-1} \\
D_{\mu} \Phi \longrightarrow\left(D_{\mu} \Phi\right)^{\prime}=\left(\partial_{\mu}-i g \frac{\vec{\tau}}{2} \cdot \vec{B}_{\mu}\right) \Phi^{\prime} \\
G_{\mu \nu}^{i} \longrightarrow G_{\mu \nu}^{i \prime}=\partial_{\mu} B_{\nu}^{i}-\partial_{\nu} B_{\mu}^{i}+g \epsilon^{i j k} B_{\mu}^{j} B_{\nu}^{k}
\end{gathered}
$$

Then we have the Lagrangian:

$$
\mathcal{L}=\left(D_{\mu} \Phi\right)^{\prime \dagger}\left(D^{\mu} \Phi\right)^{\prime}+\frac{\mu^{2}}{2}(v+\eta)^{2}-\frac{\lambda}{4}(v+\eta)^{4}-\frac{1}{4} G_{\mu \nu}^{i \prime} G^{i \prime \mu \nu}
$$

The mass term of gauge bosons comes from the part of the kinetic term:

$$
\frac{g^{2}}{8}(0, v) \cdot\left(\vec{\tau} \cdot \vec{B}_{\mu} \vec{\tau} \cdot \vec{B}^{\mu}\right) \cdot\left(\begin{array}{l}
0 \\
v
\end{array}\right)=\frac{1}{2}\left(\frac{g v}{2}\right)^{2} \vec{B}_{\mu} \cdot \vec{B}^{\mu}
$$

In this parameterization, $\vec{\xi}$ s are the Nambu-Goldstone bosons; $\eta$ is the Higgs field. From the Lagrangian, $m_{\eta}=\sqrt{2} \mu$. Therefore, through the Higgs mechanism, the Nambu-Goldstone is absorbed into the longitudinal component of the gauge bosons and which become massive. In the next section, we investigate the Standard Model as a gauge theory. 


\subsection{The Standard Model of Electroweak Inter- actions}

The electroweak model (EW) is a unified theory to describe all electromagnetic and weak phenomena by one single Lagrangian. However, the biggest caveat is the requirement for the masses of $W / Z$ vector bosons. In the EW model, all force carriers are hypothesized to be massless. This hypothesis is correct in case of the photon because electromagnetic interactions are long-range forces. On the other hand, experiments indicate weak interactions to be short-range. Therefore, the force carriers of weak interactions $W / Z$ should be massive instead. The crisis was solved by introducing the idea of the Spontaneous Symmetry Breaking (SSB) through the Brout-Englert-Higgs (BEH) mechanism, commonly referred as the Higgs mechanism, [2] under which the photon remains massless and the $W / Z$ bosons acquire their masses by "eating" Nambu-Goldstone bosons along with the existence of the Higgs field.

It must be clarified that the Standard Model nowadays is referred to the unified theory of strong, electrodynamic, and weak interactions. However, the scope of this thesis is to study the model of right-handed neutrinos at electroweak scale. So we limit ourselves in the unified theory of electrodynamic and weak interactions. Then we refer the standard model of electroweak interactions as the Standard Model.

\subsubsection{The Gauge Group of the Standard Model}

The Standard Model is the unified theory of electromagnetic and weak interactions, so the gauge group $G_{S M}$ should be the combination of $S U(2)_{L}$ and $U(1)$. After the spontaneous symmetry breaking, $G_{S M} \longrightarrow U(1)_{e m}$, with electric charge $Q$ is the operator of $U(1)_{e m}$. 
Considering the leptons which are the left-handed doublets under $S U(2)_{L}$ :

$$
l_{L}=\left(\begin{array}{c}
\nu_{e} \\
e^{-}
\end{array}\right)_{L} .
$$

We know that the electric charge of neutrinos and electrons are $0,-1$, respectively. It means that

$$
Q\left(\nu_{e L}\right)=0 ; \quad Q\left(e_{L}^{-}\right)=-1
$$

Also, $\nu_{e L}, e_{L}^{-}$are the two members of the $S U(2)_{L}$ doublet. Thus they should have weak charges of

$$
T_{3 L}\left(\nu_{e L}\right)=\frac{1}{2} ; \quad T_{3 L}\left(e_{L}^{-}\right)=-\frac{1}{2} .
$$

If $Q, T_{3 L}$ satisfy the formula

$$
Q=T_{3 L}+\frac{Y}{2}
$$

then $Y=-1$ for both $\nu_{e L}$ and $e_{L}^{-}$. Eq. (1.4.4) is Gell-mann - Nishinjima formula. It means that $Y$ commutes with $T_{L}^{i}$. So the electric charge $Q$ can be seen as a linear combination of the neutral $S U(2)_{L}$ generator $T_{3 L}$ and the hyper-charge operator $\frac{Y}{2}$ of the group $U(1)$. Then we call $U(1)_{Y}$ due to this matter. We have

$$
\left[Y, T_{L}^{i}\right]=0
$$

The gauge group of the Standard Model is:

$$
G_{S M}=S U(2)_{L} \times U(1)_{Y}
$$

In the Standard Model, $e_{R}^{-}, \nu_{e R}$ are both singlets under $S U(2)_{L}$ due to the lack of $\mathrm{V}+\mathrm{A}$ interactions. Applying Eq.(1.4.4) formula then

$$
\begin{aligned}
& \frac{Y}{2}\left(e_{R}^{-}\right)=Q\left(e_{R}^{-}\right)-T_{3 L}\left(e_{R}^{-}\right)=-1-0=-1 \\
& \frac{Y}{2}\left(\nu_{R}\right)=Q\left(\nu_{R}\right)-T_{3 L}\left(\nu_{R}\right)=0
\end{aligned}
$$


It means that $\nu_{R}$ has zero quantum numbers under both $S U(2)_{L}$ and $U(1)_{Y}$. In other word, the right-handed neutrino has no interaction with the gauge fields of both groups. The consequence of the sterile nature of the right-handed neutrinos in the Standard Model will be discussed in details in the next chapter.

\subsubsection{The Standard Model as a Gauge Theory}

Under the gauge group of $S U(2)_{L} \times U(1)_{Y}$, the covariant derivative is

$$
\partial_{\mu} \longrightarrow D_{\mu}=\partial_{\mu}-i g \vec{T} \vec{W}_{\mu}-i g^{\prime} \frac{Y}{2} B_{\mu}
$$

Here $\vec{W}_{\mu}, B_{\mu}$ are the gauge fields of the groups $S U(2)_{L}, U(1)_{Y}$, respectively. When the covariant derivative acts on different fields, the operators $\vec{T}, \frac{Y}{2}$ generate the corresponding eigenvalues to the fields. Under the gauge group $G_{S M}$ of the Standard Model, quarks and leptons transform as:

$$
\begin{aligned}
& l_{L}=\left(\begin{array}{c}
\nu_{e L} \\
e_{L}
\end{array}\right) \sim(2,-1) ; \quad e_{R} \sim(1,-2) ; \quad \nu_{R} \sim(1,0) \\
& q_{L}=\left(\begin{array}{c}
u_{L} \\
d_{L}
\end{array}\right) \sim\left(2, \frac{1}{3}\right) ; \quad u_{R} \sim\left(1, \frac{4}{3}\right) ; \quad d_{R} \sim\left(1,-\frac{2}{3}\right)
\end{aligned}
$$

Then we have the covariant derivatives for these fermionic fields:

$$
\begin{aligned}
D_{\mu} l_{L} & =\left(\partial_{\mu}-i g \vec{T} \cdot \vec{W}_{\mu}+i g^{\prime} \frac{1}{2} B_{\mu}\right) l_{L} \\
D_{\mu} q_{L} & =\left(\partial_{\mu}-i g \vec{T} \cdot \vec{W}_{\mu}-i g^{\prime} \frac{1}{6} B_{\mu}\right) q_{L} \\
D_{\mu} \nu_{R} & =\partial_{\mu} \nu_{R} \\
D_{\mu} e_{R} & =\left(\partial_{\mu}+i g^{\prime} B_{\mu}\right) e_{R} \\
D_{\mu} u_{R} & =\left(\partial_{\mu}-i g^{\prime} \frac{2}{3} B_{\mu}\right) u_{R} \\
D_{\mu} d_{R} & =\left(\partial_{\mu}+i g^{\prime} \frac{1}{3} B_{\mu}\right) d_{R} .
\end{aligned}
$$


So the Lagrangian for fermions is:

$$
\begin{aligned}
\mathcal{L}_{\text {Fermions }} & =\bar{l}_{L} i \gamma^{\mu} D_{\mu} l_{L}+\bar{e}_{R} i \gamma^{\mu} D_{\mu} e_{R}+\bar{\nu}_{R} i \gamma^{\mu} D_{\mu} \nu_{R} \\
& +\bar{q}_{L} i \gamma^{\mu} D_{\mu} q_{L}+\bar{u}_{R} i \gamma^{\mu} D_{\mu} u_{R}+\bar{d}_{R} i \gamma^{\mu} D_{\mu} d_{R}
\end{aligned}
$$

The Lagrangian for the gauge sector:

$$
\begin{aligned}
\mathcal{L}_{\text {Gauge }} & =-\frac{1}{4} G_{\mu \nu}^{i} G^{i \mu \nu}-\frac{1}{4} B_{\mu \nu} B^{\mu \nu} \\
G_{\mu \nu}^{i} & =\partial_{\mu} W_{\nu}^{i}-\partial_{\nu} W_{\mu}^{i}+g \varepsilon^{i j k} W_{\mu}^{j} W_{\nu}^{k} \\
B_{\mu \nu} & =\partial_{\mu} B_{\nu}-\partial_{\nu} B_{\mu}
\end{aligned}
$$

Here, $G_{\mu \nu}^{i}$, and $B_{\mu \nu}$ are the field strength tensors. $\varepsilon^{i j k}$ is the structure constant of the non-Abelian group $S U(2)$.

From the Gell-Mann-Nishijima formula Eq.(1.4.4), the gauge field associated with the electric charge $Q$ must be the combination of $W_{\mu}^{3}$ and $B_{\mu}$. The field is actually the photon field $A_{\mu}$. When $S U(2)_{L} \times U(1)_{Y} \longrightarrow U(1)_{e m}$, both $S U(2)_{L}$ and $U(1)_{Y}$ have to be broken spontaneously. There must exist an appropriate scalar field responsible for that. To break $S U(2)_{L}$ and $U(1)_{Y}$ altogether, then it must have the $S U(2)_{L}$ and $U(1)_{Y}$ quantum numbers. On the other hand, $W^{ \pm}, W^{3}$ shall acquire their masses through this procedure. We need three Nambu-Goldstone bosons. Essentially, the scalar has to have more than three degrees of freedom. The simplest choice is a complex doublet, $\Phi$.

$$
\Phi=\left(\begin{array}{c}
\phi^{+} \\
\phi^{0}
\end{array}\right) \sim(2,1)
$$

To avoid a charged vacuum, the VEV of the Higgs field is chosen as $\left\langle\phi^{+}\right\rangle=0$ and $\left\langle\phi^{0}\right\rangle=v / \sqrt{2}$. The Lagrangian for the Higgs field is

$$
\begin{array}{r}
\mathcal{L}_{\text {Higgs }}=\frac{1}{2}\left(D_{\mu} \Phi D^{\mu}\right)^{\dagger} \Phi-V\left(\Phi^{2}\right) \\
D_{\mu} \Phi=\left(\partial_{\mu}-i g \vec{T} \cdot \vec{W}_{\mu}-i g^{\prime} \frac{Y_{H}}{2} B_{\mu}\right) \Phi \\
V\left(\Phi^{\dagger} \Phi\right)=-\frac{1}{2} \mu^{2}\left(\Phi^{\dagger} \Phi\right)^{2}+\frac{\lambda}{4}\left(\Phi^{\dagger} \Phi\right)^{2}
\end{array}
$$


$\star$ If $\mu^{2}>0$, the potential will have the parabolic shape as in Fig 1.7. The vacuum will be unique and symmetric, then the spontaneous symmetry breaking cannot happen.

$\star$ While if $\mu^{2}<0$, the ground state occurs at $\Phi^{2}=-\frac{1}{2} \mu^{2} / \lambda$. The potential $V\left(\Phi^{2}\right)$ has the famous "Mexican hat" shape as in Figure 1.8. It means that the theory has an infinite number of vacua along the circles $<\Phi>^{2}=\mu^{2} / \lambda$. If, for some reason, one vacuum is chosen, then the symmetry of the ground state is broken spontaneously.

$$
S U(2)_{L} \times U(1)_{Y} \rightarrow U(1)_{e m}
$$

It is convenient to represent $\Phi$ in terms of four real scalar fields $H, \zeta_{1}, \zeta_{2}$, and $\zeta_{3}$

$$
\Phi=\left(\begin{array}{c}
\phi^{+} \\
\phi^{0}
\end{array}\right)=\exp \left(\frac{i}{v} \sum \zeta_{i} T_{i}\right)\left(\begin{array}{c}
0 \\
\frac{1}{\sqrt{2}}(v+H)
\end{array}\right) .
$$

Then all these fields have zero $V E V$ :

$$
<\zeta_{i}>=<H>=0
$$

In unitary gauge, $\zeta_{i}$ are three Nambu-Goldstone bosons which will be absorbed by $W^{ \pm} / Z$. $H$ is the physical Higgs field. Let us consider the $E W$ model under the unitary transformation

$$
U=\exp \left(-\frac{i}{v} \sum \zeta_{i} T_{i}\right)
$$


The transformation of the component fields as following:

$$
\begin{gathered}
\Phi \longrightarrow \Phi^{\prime}=U \Phi=\left(\begin{array}{c}
0 \\
\frac{1}{\sqrt{2}}(v+H)
\end{array}\right) ; \\
l_{L} \longrightarrow l_{L}^{\prime}=U l_{L} ; \quad e_{R} \rightarrow e_{R}^{\prime}=U e_{R} ; \\
B_{\mu} \longrightarrow B_{\mu}^{\prime}=U B_{\mu} ; \\
\vec{T} \overrightarrow{W_{\mu}} \longrightarrow \vec{T} \vec{W}_{\mu}^{\prime}=U \vec{T} \overrightarrow{W_{\mu}} U^{-1}-\frac{i}{g}\left(\partial_{\mu} U\right) U^{-1} ; \\
D_{\mu} \Phi \longrightarrow\left(D_{\mu} \Phi\right)^{\prime}=\left(\partial_{\mu}-i g \vec{T} \cdot \vec{W}_{\mu}^{\prime}-i g^{\prime} \frac{Y_{H}}{2} B_{\mu}^{\prime}\right)\left(\begin{array}{c}
0 \\
\frac{1}{\sqrt{2}}(v+H)
\end{array}\right)
\end{gathered}
$$

Note that:

$$
\begin{aligned}
\vec{T} \cdot \vec{W}_{\mu}^{\prime} & =\frac{1}{2}\left(\tau_{1} W_{1 \mu}^{\prime}+\tau_{2} W_{2 \mu}^{\prime}+\tau_{3} W_{3 \mu}^{\prime}\right) \\
& =\frac{1}{\sqrt{2}}\left(\tau_{+} W_{\mu}^{+\prime}+\tau_{-} W_{\mu}^{\prime \dagger}\right)+\frac{1}{2} \tau_{3} W_{3 \mu}^{\prime}
\end{aligned}
$$

with the definition $\tau_{ \pm}=\frac{1}{2}\left(\tau_{1} \pm i \tau_{2}\right)$ and $W_{\mu}^{\prime}=\frac{1}{\sqrt{2}}\left(W_{1 \mu}^{\prime}-i W_{2 \mu}^{\prime}\right)$.

The kinetic part of the scalar fields:

$$
\begin{aligned}
\left(D_{\mu} \Phi\right)^{\prime \dagger}\left(D_{\mu} \Phi\right) & =\frac{1}{4} g^{2}(v+H)^{2} W_{\mu}^{\prime \dagger} W^{\mu \prime}+\frac{1}{2}\left[\partial_{\mu} H \partial^{\mu} H+\frac{1}{4}(v+H)^{2}\left(g W_{3 \mu}^{\prime}-g^{\prime} B_{\mu}^{\prime}\right)^{2}\right] \\
& =\frac{1}{4} g^{2} v^{2} W_{\mu}^{\prime \dagger} W^{\mu \prime}+\frac{1}{8} v^{2}\left(g W_{3 \mu}^{\prime}-g^{\prime} B_{\mu}^{\prime}\right)^{2}+ \\
& +\frac{1}{2} \partial_{\mu} H \partial^{\mu} H+\frac{1}{4}\left(2 v H+H^{2}\right)\left[g^{2} W_{\mu}^{\prime \dagger} W^{\mu \prime}+\frac{1}{2}\left(g W_{3 \mu}^{\prime}-g^{\prime} B_{\mu}^{\prime}\right)^{2} .\right.
\end{aligned}
$$

So the mass of the charged vector boson $M_{W}=\frac{1}{2} g v$. While the third component $W_{3 \mu}^{\prime}$ and $B_{\mu}^{\prime}$ can mix together through the mass mixing matrix:

$$
M=\frac{v^{2}}{4}\left(\begin{array}{cc}
g^{2} & -g g^{\prime} \\
-g g^{\prime} & g^{\prime 2}
\end{array}\right)
$$

After diagonalizing, there exist two mass eigenstates:

$$
\begin{aligned}
& Z_{\mu}=\cos \theta_{W} W_{\mu}^{3 \prime}-\sin \theta_{W} B_{\mu}^{\prime}, \\
& A_{\mu}=\sin \theta_{W} W_{\mu}^{3 \prime}+\cos \theta_{W} B_{\mu}^{\prime} ;
\end{aligned}
$$


here

$$
\cos \theta_{W}=\frac{g}{\sqrt{g^{2}+g^{\prime 2}}} ; \quad \sin \theta_{W}=\frac{g^{\prime}}{g^{2}+g^{\prime 2}} .
$$

The eigenvalues of the mass matrix correspond to the masses $\gamma, Z$ are:

$$
M_{\gamma}^{2}=0 ; M_{Z}^{2}=\frac{1}{4}\left(g^{2}+g^{2}\right) v^{2} .
$$

It is realized that

$$
\frac{M_{W}^{2}}{M_{Z}^{2}}=\frac{g^{2}}{g^{2}+g^{\prime 2}}=\cos ^{2} \theta_{W}
$$

Consequently, $M_{W}=M_{Z} \cos \theta_{W}$. This is the first prediction of the Standard Model of Electroweak interactions. It shows the correlation between three quantities $m_{W}, m_{Z}$, and $\theta_{W}$, and predicts the third one if the other two could be measured. Moreover, it leads to an important parameter of the precision measurements of the Standard Model:

$$
\rho=\frac{M_{W}^{2}}{M_{Z}^{2} \cos ^{2} \theta_{W}}=1 .
$$

The $\rho$-parameter represents the ratio of the relative strength of the neutral current to the charged current in weak interactions.

In the inverse representation,

$$
\begin{aligned}
W_{3 \mu}^{\prime} & =\sin \theta_{W} A_{\mu}+\cos \theta_{W} Z_{\mu} \\
B_{\mu}^{\prime} & =\cos \theta_{W} A_{\mu}-\sin \theta_{W} Z_{\mu}
\end{aligned}
$$

Then,

$$
\begin{aligned}
i g T_{3} W_{\mu}^{3 \prime}+i g^{\prime} \frac{Y}{2} B_{\mu}^{\prime} & =i\left[g T_{3}\left(\cos \theta_{W} Z_{\mu}+\sin \theta_{W} A_{\mu}\right)+g^{\prime} \frac{Y}{2}\left(-\sin \theta_{W} Z_{\mu}+\cos \theta_{W} A_{\mu}\right)\right] \\
& =i \sqrt{g^{2}+g^{\prime 2}}\left(T_{3}-\sin ^{2} \theta_{W} Q\right) Z_{\mu}+i g \sin \theta_{W} Q A_{\mu} .
\end{aligned}
$$

In the interaction of $l_{L}, e_{R}$ with the photon $A_{\mu}$, we have:

$$
\bar{\psi}_{L} i\left(-i \gamma^{\mu} A_{\mu} g \sin \theta_{W} Q\right) \psi_{L}=\bar{\psi}_{L} \gamma^{\mu} \psi_{L}(e Q) A_{\mu}
$$


It leads to another prediction

$$
e=g \sin \theta_{W}
$$

While looking into the interaction of leptons and charge gauge bosons $W^{ \pm}$in the low energy approximation, it is expressed as:

$$
\frac{g^{2}}{2} J_{\mu}^{+} \frac{1}{q^{2}-m_{W}^{2}} J^{-\mu} \sim-\frac{g^{2}}{2 m_{W}^{2}} J_{\mu}^{+} J^{-\mu}=-4 \frac{G_{F}}{\sqrt{2}} J_{\mu}^{+} J^{-\mu} .
$$

So, there exists a relationship between the Fermi constant $G_{F}$ and the mass of $W$ boson:

$$
\frac{G_{F}}{\sqrt{2}}=\frac{g^{2}}{8 m_{W}^{2}}
$$

\subsubsection{Fermion Masses}

Besides giving masses to the $W / Z$, the Higgs field is also responsible for generating the masses of fermions via the Yukawa interaction.

- For leptons, the Yukawa interaction term has a form of

$$
\mathcal{L}_{Y u k}=g_{Y} \bar{l}_{L} \Phi e_{R}+h . c,
$$

which gives the mass term of leptons when $\Phi$ develops VEV

$$
\begin{aligned}
& g_{Y}\left(\begin{array}{ll}
\bar{\nu}_{L} & \bar{e}_{L}
\end{array}\right)\left(\begin{array}{c}
0 \\
\frac{v}{\sqrt{2}}
\end{array}\right) e_{R}+\text { h.c. } \\
\Rightarrow & m_{e}=g_{Y} \frac{v}{\sqrt{2}} .
\end{aligned}
$$

- For quarks, it needs to introduce the adjunct representation of the Higgs field $\tilde{\Phi}=i \tau_{2} \Phi^{\star}$. The VEV of $\tilde{\Phi}$ :

$$
<\tilde{\Phi}>=\left(\begin{array}{c}
\frac{v}{\sqrt{2}} \\
0
\end{array}\right)
$$


Then the Yukawa Lagrangian for quarks is

$$
\begin{array}{r}
\mathcal{L}_{Y u k}=g_{u} \bar{q}_{L} \tilde{\Phi} u_{R}+g_{d} \bar{q}_{L} \Phi d_{R}+\text { h.c. } \\
\Rightarrow m_{u}=g_{u} \frac{v}{\sqrt{2}}, \quad m_{d}=g_{d} \frac{v}{\sqrt{2}} .
\end{array}
$$

All the couplings $g_{Y}, g_{u}, g_{d}$ are free parameters determined from experiments. The Standard Model can not give any prediction about the values of these parameters. In this description of the Standard Model, only one family of fermions is used to illustrate the SM structure. When taking into account three families of leptons and quarks, one obtains the mixing among leptons and quarks themselves.

In general,

$\diamond$ The mass matrix of the charged leptons is:

$$
m_{l}=\frac{v}{\sqrt{2}}\left(\begin{array}{ccc}
g_{e e} & g_{e \mu} & g_{e \tau} \\
g_{\mu e} & g_{\mu \mu} & g_{\mu \tau} \\
g_{\tau e} & g_{\tau \mu} & g_{\tau \tau}
\end{array}\right)
$$

All $g_{Y} \mathrm{~s}$ are independent complex parameters. The mass eigenstates are achieved after diagonalizing $m_{l}$ matrix.

$\diamond$ As similarly for quarks:

$$
m_{u}=\frac{v}{\sqrt{2}}\left(\begin{array}{ccc}
g_{u u} & g_{u c} & g_{u t} \\
g_{c u} & g_{c c} & g_{c t} \\
g_{t u} & g_{t c} & g_{t t}
\end{array}\right) ; \quad m_{d}=\frac{v}{\sqrt{2}}\left(\begin{array}{ccc}
g_{d d} & g_{d s} & g_{d b} \\
g_{s d} & g_{s s} & g_{s b} \\
g_{b d} & g_{b s} & g_{b b}
\end{array}\right)
$$

\subsubsection{Summary}

To sum up, the Lagrangian of the Standard Model can be written as:

$$
\mathcal{L}_{\text {SM }}=\mathcal{L}_{\text {Fermions }}+\mathcal{L}_{\text {Gauge }}+\mathcal{L}_{\text {Higgs }}+\mathcal{L}_{\text {Yukawa }},
$$

with the expressions of partial Lagrangian shown separately in Eqs: (1.4.11), (1.4.12), (1.4.14), while

$$
\mathcal{L}_{\text {Yukawa }}=g_{Y} \bar{l}_{L} \Phi e_{R}+g_{u} \bar{q}_{L} \tilde{\Phi} u_{R}+g_{d} \bar{q}_{L} \Phi d_{R}+\text { h.c. }
$$




\section{Chapter 2}

\section{Neutrino masses: Facts, Origins, And Mechanisms}

The history of neutrinos started with the proposal of W. Pauli to save the energy conservation law in the $\beta$-decay process.

$$
M(A, Z) \rightarrow D(A, Z+1)+e^{-}
$$

Experiments had indicated that the energy spectrum of $\beta$-decay process was continuous rather than discrete as predicted by two-body decay. Moreover, if there were only two particles in the final states, then it was difficult to explain in terms of the angular momentum conservation. The puzzles were solved by Pauli's particle, which is a neutral and spin $\frac{1}{2}$ called "neutron". To distinguish to the neutron, Fermi named it "neutrino" which means neutral and very small. In Fermi theory of 4-fermion interactions, $\beta$-decay can be presented as 


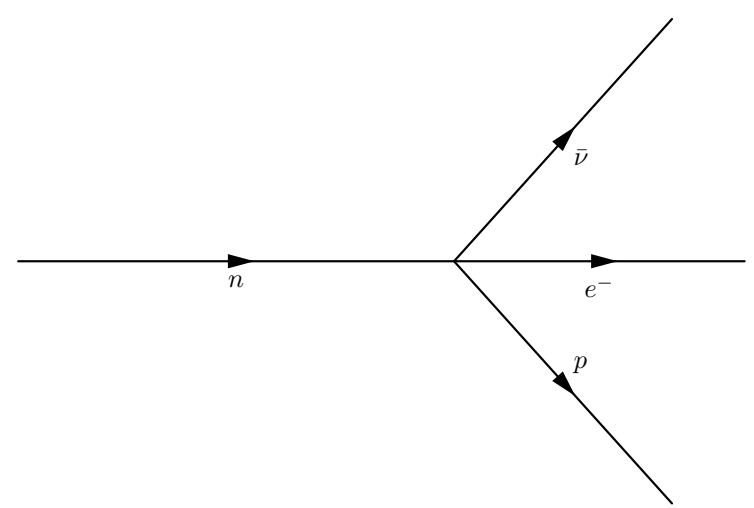

Figure 2.1. Fermi 4-fermion interaction model to describe $\beta$-decay

As discussed in the previous section, the Standard Model consists of three generations of leptons. One also has three corresponding neutrinos, $\nu_{e}, \nu_{\mu}, \nu_{\tau}$. In the framework of the Standard Model, these neutrinos are massless. However, we shall discuss the evidence for the non-zero masses of neutrinos, the different types of neutrino masses, and a mechanism to generate neutrinos masses.

\subsection{Neutrino Oscillations}

Over the last couple decades, neutrino oscillations were observed in different experiments using solar-neutrinos, reactor neutrinos, atmospheric neutrinos, and accelerator neutrinos [3]. These results implied the non-zero $\Delta m^{2}$ of the neutrino masses. In this section we present a brief theoretical background of neutrino oscillations.

\subsubsection{Mass \& Flavor Eigenstates}

To understand neutrino oscillations, we discuss the concepts of flavor and mass eigenstates of neutrinos. Lets denote the gauge eigenstates by

$$
l_{L}^{0}=\left(e_{L}^{0}, \mu_{L}^{0}, \tau_{L}^{0}\right), \quad \nu_{L}^{0}=\left(\nu_{e L}^{0}, \nu_{\mu L}^{0}, \nu_{\tau L}^{0}\right),
$$


and the mass eigenstates by

$$
l_{L}=\left(e_{L}, \mu_{L}, \tau_{L}\right), \quad \nu_{L}=\left(\nu_{1 L}, \nu_{2 L}, \nu_{3 L}\right)
$$

The two representations relate to each other through mixing matrices $U_{l}, U_{\nu}$ for the leptons and the neutrinos, respectively.

$$
l_{L}^{0}=U_{l} l_{L} ; \quad \nu_{L}^{0}=U_{\nu} \nu_{L}
$$

Let us consider the charged current interaction:

$$
\mathcal{L}_{c}=g \bar{l}_{L}^{0} \gamma_{\mu} \nu_{L}^{0} W^{\mu-}+\text { h.c. }
$$

Then in the mass eigenstate representation:

$$
\mathcal{L}_{c}=g \bar{l}_{L} \gamma_{\mu} U_{l}^{-1} U_{\nu} \nu_{L} W^{\mu-}+\text { h.c. }
$$

Define

$$
\left(\nu_{e L}, \nu_{\mu L}, \nu_{\tau L}\right)=U^{l} \nu_{L}
$$

here $U^{\text {lepton }}=U_{l}^{-1} U_{\nu}$. The charged current interaction can be expressed as:

$$
\mathcal{L}_{c}=g\left(\bar{e}_{L}, \bar{\mu}_{L}, \bar{\tau}_{L}\right) \gamma_{\nu}\left(\begin{array}{c}
\nu_{e L} \\
\nu_{\mu L} \\
\nu_{\tau L}
\end{array}\right) W^{\mu-}+h . c .
$$

\subsubsection{Neutrino Oscillations in Vacuum}

Generally, quantum oscillation is the phenomenon where a particle in a reaction is not identical with the one that was produced some time before. Assuming that a neutrino with flavor $\alpha$ is produced at $t=0$.

$$
\left|\nu_{\alpha}(0)>=\sum_{i} U_{\alpha i}^{l}\right| \nu_{i}(0)>
$$


The probability of $\nu_{\alpha} \rightarrow \nu_{\beta}$ after traveling a distance $L$ is

$$
\begin{aligned}
P_{\nu_{\alpha} \rightarrow \nu_{\beta}} & =\mid\left\langle\nu_{\alpha}\left|\nu_{\beta}>\right|^{2}\right. \\
& =\sum_{i, j} U_{\alpha_{i}} U_{\beta i}^{\star} U_{\alpha j}^{\star} U_{\beta j} e^{-i \frac{m_{i}^{2}-m_{j}^{2}}{2 E} L}
\end{aligned}
$$

Here, $U_{a b}$ are the elements of the mixing matrix of leptons, so-called $U_{P M N S}$ in the names of Pontocorve, Maki, Nakagawa, and Sakara. In general, $U_{P M N S}$ depends on the mixing between three families of leptons and a parameter responsible for $C P$ violation, $\delta_{C P}$. One possible parametrization of $U_{P M N S}$ is:

$U_{P M N S}=\left(\begin{array}{lll}c_{12} c_{13} & s_{12} c_{13} & s_{13} e^{-i \delta_{C P}} \\ -s_{12} c_{23}-c_{12} s_{13} s_{23} e^{i \delta_{C P}} & c_{12} c_{23}-s_{12} s_{13} s_{23} e^{i \delta_{C P}} & c_{13} s_{23} \\ s_{12} s_{23}-c_{12} s_{13} c_{23} e^{i \delta_{C P}} & -c_{12} s_{23}-s_{12} s_{13} c_{23} e^{i \delta_{C P}} & c_{13} c_{23}\end{array}\right)$ (2.1.10) where $c_{i j}=\cos \theta_{i j}, s_{i j}=\sin \theta_{i j}$, and $\theta_{i j}=[0, \pi / 2]$.

Putting the speed of light $c$ and the Planck constant $\hbar$ into the formula, we have the transition probability of $\nu_{\alpha} \rightarrow \nu_{\beta}[4]$ :

$$
\begin{aligned}
P_{\nu_{\alpha} \rightarrow \nu_{\beta}} & =\delta_{\alpha \beta}-4 \sum_{i<j} \operatorname{Re}\left[U_{\alpha i} U_{\beta i}^{\star} U_{\alpha J}^{\star} U_{\beta j}\right] \sin ^{2}\left(X_{i j}\right) \\
& +2 \sum_{i<j} \operatorname{Im}\left[U_{\alpha i} U_{\beta i}^{\star} U_{\alpha J}^{\star} U_{\beta j}\right] \sin \left(2 X_{i j}\right)
\end{aligned}
$$

here $X_{i j}=1.27 \frac{\Delta m_{j i}^{2}(\mathrm{eV})^{2}}{4 E(G e V)} L(\mathrm{~km}), E$ is the energy of the neutrino source, $L$ is the distance from the source.

For example, in the case of two flavors, $\nu_{e}, \nu_{\mu}$, the probability for $\nu_{\mu}$ to be converted into $\nu_{e}$ is

$$
P\left(\nu_{\mu} \rightarrow \nu_{e}\right)=\sin ^{2} 2 \theta \sin ^{2}\left(1.27 \frac{\Delta m_{21}^{2}(\mathrm{eV})^{2}}{4 E(G e V)} L(k m)\right)
$$

Therefore, neutrino oscillations require the non-zero mixing between mass eigenstates and non-zero in mass difference as indicated in Eq. (2.1.12). In other words, the existence of neutrino oscillations proves that at least two of the three neutrino flavors have to have non-zero masses. 


\subsubsection{Experimental Results}

Neutrino oscillations indicate that two out three neutrino flavors must have non-zero and non-degenerate masses. Nevertheless, the oscillations do not imply the order of mass of these flavors, because the probability of $\nu_{\mu} \rightarrow \nu_{e}$ depends on the $\sin ^{2}\left(\Delta m_{21}^{2} \ldots\right)$. Generally, the mass spectrum of neutrinos can be classified into two orders

- Normal Hierarchy (NH): $m_{1}<m_{2}<m_{3}$

- Inverted Hierarchy (IH): $m_{3}<m_{1}<m_{2}$

Up to date, the global fit of neutrino oscillations is given in Table 2.1 [5]:

\begin{tabular}{|c|c|}
\hline parameter & best fit $\pm 1 \sigma$ \\
\hline$\Delta m_{21}^{2}\left[10^{-5} \mathrm{eV}^{2}\right]$ & $7.56_{-0.22}^{+0.26}$ \\
$\left|\Delta m_{31}^{2}\right|\left[10^{-3} \mathrm{eV}^{2}\right](\mathrm{NH})$ & $2.43_{-0.07}^{+0.05}$ \\
$\left|\Delta m_{31}^{2}\right|\left[10^{-3} \mathrm{eV}^{2}\right](\mathrm{IH})$ & $2.38_{-0.06}^{+0.06}$ \\
$\sin ^{2} \theta_{12}$ & $0.308 \pm 0.017$ \\
$\sin ^{2} \theta_{23}(N H)$ & $0.437_{-0.023}^{+0.033}$ \\
$\sin ^{2} \theta_{23}(I H)$ & $0.455_{-0.031}^{+0.039}$ \\
$\sin ^{2} \theta_{13}(N H)$ & $0.0234_{-0.0019}^{0.0020}$ \\
$\sin ^{2} \theta_{13}(I H)$ & $0.0240_{-0.0020}^{0.0019}$ \\
$\delta / \pi(\mathrm{NH})$ & $1.39_{-0.27}^{+0.38}$ \\
$\delta / \pi(\mathrm{IN})$ & $1.31_{-0.33}^{+0.29}$ \\
\hline
\end{tabular}

Table 2.1. The parameters of neutrino oscillations.

As the first solid evidence for the non-zero, tiny mass of neutrinos, the phenomenon of neutrino oscillations is one of the most important topics of neutrino 
physics. Other important topics have been widely investigated such as the effect of matter on neutrino oscillations, the oscillations of solar neutrinos, etc. However in this thesis, we just present the neutrino oscillations in the vacuum to show the necessity of building a model to explain the non-zero mass of neutrinos.

\subsection{Neutrinos Mass}

As discussed in the last section, neutrino oscillations imply that neutrinos have non-zero masses. Unlike charged leptons and quarks, neutrinos can have two types of masses, Dirac mass and Majorana mass. We start out by introducing three generations of right-handed neutrinos corresponding to three families of leptons. These right-handed neutrinos are singlets under the $G_{S M}$ group.

\subsubsection{Dirac mass}

The Dirac mass of neutrinos comes from the Lagrangian:

$$
\mathcal{L}_{D}=g_{\nu_{e}} \bar{l}_{e L} \tilde{\phi} \nu_{e R}+\text { h.c. }
$$

Here we use one family for simplicity again. When $\tilde{\phi}=i \tau_{2} \phi^{*}$ acquires VEV, $<\tilde{\phi}>=\left(\begin{array}{c}\frac{v}{\sqrt{2}} \\ 0\end{array}\right)$, the Lagrangian contains the terms:

$$
g_{\nu_{e}} \frac{v}{\sqrt{2}} \bar{\nu}_{e L} \nu_{e R}+\text { h.c. }
$$

So $m_{\nu_{e}}^{D}=g_{\nu_{e}} \frac{v}{\sqrt{2}}$, here $v=246 \mathrm{GeV}$. In the most recent results from the Planck satellite, the upper limit of the neutrinos mass is $m_{\nu}<0.23 \mathrm{eV}$ [6]. If the mass of neutrinos comes only from the Dirac type, the coupling $g_{\nu}$ is $\sim O\left(10^{-11}\right)$. There is no principle to prevent such a small coupling, but it seems an unnaturally small scale. One alternative way to achieve such a small mass is to generate it through a dynamical mechanism either by a symmetry or through new concepts such as extra dimensions. 


\subsubsection{Majorana mass}

Unlike charged leptons, neutrinos are their anti-neutrinos due to their neutral nature. They then can have Majorana mass in the form of $\nu_{L}^{T} \sigma_{2} \nu_{L}$ or $\nu_{R}^{T} \sigma_{2} \nu_{R}$.

$\diamond$ In the Standard Model, $\nu_{L}^{T} \sigma_{2} \nu_{L}$ comes from the bilinear $l_{L}^{T} \sigma_{2} l_{L}$, which has hypercharge $Y=-1$. It needs to couple to a scalar with the hypercharge +1 in order to preserve the gauge invariance. The appropriate choice is a triplet Higgs $\vec{\Delta}=\left(\Delta^{++}, \Delta^{+}, \Delta^{0}\right)$. Then the Majorana mass term comes from:

$$
\mathcal{L}_{M}=i g_{\Delta} l_{L}^{T} \sigma_{2} \vec{\tau} \cdot \vec{\Delta} l_{L}
$$

Here,

$$
\vec{\tau} \cdot \vec{\Delta}=\left(\begin{array}{cc}
\Delta^{+} / \sqrt{2} & \Delta^{++} \\
\Delta^{0} & -\Delta^{+} / \sqrt{2}
\end{array}\right)
$$

Then

$$
\mathcal{L}_{M}=g_{\Delta}\left(-e_{L}^{T} \sigma_{2} \frac{\Delta^{+}}{\sqrt{2}} \nu_{L}+\nu_{L}^{T} \sigma_{2} \Delta^{0} \nu_{L}-e_{L}^{T} \sigma_{2} \Delta^{++} e_{L}-\nu_{L}^{T} \sigma_{2} \frac{\Delta^{+}}{\sqrt{2}} e_{L}\right) .
$$

With the choice of $\mathrm{VEV}<\Delta>=\left(0,0, v_{\Delta}\right)$, then the Majorana mass of neutrinos is $m_{\nu}^{M}=g_{\Delta} v_{\Delta}$. Again, the smallness of neutrinos mass requires $v_{\Delta}$ to be relatively small. This leads to a well-known Majoron problem [7].

$\diamond$ On the other hand, $\nu_{R}^{T} \sigma_{2} \nu_{R}$ is a singlet under the SM gauge group. The simplest choice for the extended scalar is a singlet $\phi_{s}$.

\subsection{The Seesaw Mechanism}

\subsubsection{The Mechanism}

The more elegant way to generate the mass of neutrinos is the Seesaw Mechanism [8]. Once we add a right-handed neutrino $\nu_{R}$ per family of fermions, the leptonic sector is given by:

$$
\left(\begin{array}{l}
\nu \\
e
\end{array}\right)_{L}, \quad e_{R}, \quad \nu_{R}
$$


The Lagrangian is

$$
\mathcal{L}_{I}=g_{D} \bar{l}_{L} \sigma_{2} \phi^{\star} \nu_{R}+\frac{M_{R}}{2} \nu_{R}^{T} C \nu_{R}+\text { h.c. }
$$

Introducing Majorana spinors:

$$
\nu_{M}=\nu_{L}+C \bar{\nu}_{L}^{T}, \quad N_{M}=\nu_{R}+C \bar{\nu}_{R}^{T}
$$

Then the Lagrangian becomes:

$$
\mathcal{L}_{I}=\frac{1}{2} m_{D}\left(\bar{\nu}_{M} N_{M}+\bar{N}_{M} \nu_{M}\right)+\frac{M_{E}}{2} \bar{N}_{M} N_{M}
$$

where $m_{D}=g_{D} v$ and $v=<\phi^{0}>$ is the VEV of the neutral component of $\phi$. One has the mass matrix:

$$
\nu_{M}\left(\begin{array}{cc}
0 & m_{D} \\
m_{D} & M_{R}
\end{array}\right)
$$

In the limit $M_{R} \gg m_{D}$, the eigenvalues of the mass matrix are:

$$
\begin{aligned}
& m_{\nu} \approx \frac{m_{D}^{2}}{M_{R}}, \\
& m_{N} \approx M_{R}
\end{aligned}
$$

and the two eigenstates are:

$$
\begin{gathered}
\nu \approx \nu_{M}+\frac{m_{D}}{M_{R}} N_{M} \\
N \approx N_{M}-\frac{m_{D}}{M_{R}} \nu_{M}
\end{gathered}
$$

The constraint of the neutrino mass is $m_{\nu} \leq 0.23 \mathrm{eV}, m_{D}$ is typically at the electroweak scale $\Lambda_{E W}$, then $M_{R} \approx O\left(10^{16} \mathrm{GeV}\right)$. The seesaw here is between the scale of $M_{R}$ and electroweak scale. This is the original seesaw model. The requirement of such heavy right-handed neutrinos makes this class of seesaw models impossible to be tested at the LHC. 


\subsubsection{The Left-Right Symmetric Model}

One alternative possibility based on the seesaw mechanism is the left-right symmetric extension of the Standard Model in which Parity is a fundamental symmetry [9]. In order to break it spontaneously, one has to enlarge the gauge group. Omitting the $S U(3)_{C}$ for the purpose of simplicity, the gauge group of the minimal Left-Right model, in short LR, is

$$
G_{L R}=S U(2)_{L} \times S U(2)_{R} \times U(1)_{B-L}
$$

The electric charge is calculated by:

$$
Q=T_{3 L}+T_{3 R}+\frac{B-L}{2}
$$

All left-handed quarks and leptons have a right-handed symmetric version. The left-handed fermions are doublets under $S U(2)_{L}$ and singlets under $S U(2)_{R}$, and vice versa.

$$
\begin{aligned}
& q_{L}=\left(\begin{array}{l}
u \\
d
\end{array}\right)_{L}(2,1,1 / 3) \stackrel{\mathrm{P}}{\longleftrightarrow} q_{R}=\left(\begin{array}{l}
u \\
d
\end{array}\right)_{R}(1,2,1 / 3) \\
& l_{L}=\left(\begin{array}{l}
\nu \\
e
\end{array}\right)_{L}(2,1,-1) \stackrel{\mathrm{P}}{\longleftrightarrow} l_{R}=\left(\begin{array}{l}
\nu \\
e
\end{array}\right)_{R}(2,1,-1)
\end{aligned}
$$

So the right-handed neutrinos exist naturally. We also see that $\nu_{R}$ belongs to a doublet instead of singlet as seen in the seesaw mechanism. Again, we just use one generation here. Similarly in the gauge sector, the LR symmetry requires $W_{R}, Z_{R}$ along with $W_{L}, Z_{L}$. To generate the neutrino mass, one needs 2 scalar triplets:

$$
\Delta_{L}(3,1,2) \quad, \quad \Delta_{R}(1,3,2)
$$

The mass of the light neutrinos can be achieved by applying the seesaw mechanism. The LR symmetric model can be probed at the hadron colliders through the 
same sigh di-lepton processes: $d \bar{u} \rightarrow W_{R} \rightarrow l_{1} N_{R} \rightarrow l_{1} l_{2} W_{R}^{\star} \rightarrow l_{1} l_{2} q \bar{q}$ as proposed in [10]. In the most recent result from CMS, there is nothing to be found in the search for the heavy neutrinos and $W_{R}$ in the Left-Right symmetric model [11]. It sets a lower limit on the mass of the right-handed $W$ boson $M_{R} \geq 3 \mathrm{TeV}$.

\subsubsection{Summary of The Chapter}

The experimental results of neutrino oscillations indicate the non-zero, tiny mass of neutrinos. Though the small mass of neutrinos could be realized in the framework of the Standard Model in an ad-hoc way, it would be more attractive to explain the neutrino mass by introducing the physics beyond the Standard Model. The key answer would be lying in the nature of the mass of neutrinos. Is it just the Dirac type or the mixture of the Dirac and Majorana types? If it turns out the Dirac mass is the only source of neutrino mass, then the Standard Model could explain it with the small Yukawa coupling. And the mass of neutrinos does not give any clue about new physics. Even though we still have to look for the new symmetry underlying the smallness of the Yukawa coupling in the neutrinos sector.

On the other hand, the Majorana nature of neutrino mass is obviously the sign of the physics beyond the Standard Model. The lepton flavor violating processes such as double $\beta$ decay or like sign di-lepton are the direct evidence of the Majorana mass of neutrinos. In this case, the seesaw mechanism would be a very elegant framework for generating the small mass of neutrinos. However, the requirement of a very high mass scale make these kinds of models impossible to detect at the LHC, or near future machines. The Left-Right symmetric extension of the Standard Model would make the seesaw mechanism more predictive as it ties the high mass scale with the breaking scale of the right-handed gauge sector. Unfortunately, the most recent result from CMS sets the lower limit of $W_{R}$ up to $3 \mathrm{TeV}$. 
One may ask whether we could build a model so that the mass of the light neutrinos arises naturally through the Seesaw Mechanism, and the new particle content has a large enough production rate in order to be detected at current collider energy levels? The answer is well addressed in the Model of Right-handed neutrinos at the Electroweak scale, in short $\mathrm{EW} \nu_{R}$, and its extension. 


\section{Chapter 3}

\section{The Model of Right-Handed Neutrinos at ElectroWeak Scale}

\subsection{Motivations}

So far, the non-zero mass of neutrinos strongly indicates Physics Beyond the Standard Model. An elegant way to explain the small mass $(<e V)$ of neutrinos is through the seesaw mechanism discussed in the last chapter. The loophole in this class of models is the testability at the current running hadron colliders such as the LHC. In these models, there must exist a very high mass scale $O\left(10^{14} \mathrm{GeV}\right)$, at least, to make the see-saw work. Therefore, it is almost impossible to test these models. One interesting alternative is the model of Left-Right Symmetry which extends the Standard Model in both the particle content and the gauge structure. There are also two scales of symmetry breaking. The left-handed gauge sector is spontaneously broken at the electroweak scale while the right-handed breaking scale is only constrained in the lower bound by the experiments. Recent work of the CMS experiment on the search for heavy neutrinos and $\mathrm{W}$ bosons with right-handed couplings at the LHC put the lower bound of $M_{W_{R}} \geq 3.0 \mathrm{TeV}$ at a $95 \%$ of confidence level. So the Left-Right symmetric model is also facing a tough path ahead. 
The problem of these extensions of the Standard Model lies in the inert nature of the right-handed neutrinos because they are singlets under the SM gauge group. So one may ask if we can construct a model in which:

- The gauge group is still the same as the gauge group of the Standard Model

- The right-handed neutrinos are the one component of the doublets under the gauge group instead being singlets as in the Standard Model.

Then, in this case, the right-handed neutrinos can couple to the gauge vector bosons directly. Consequently, they can be produced and detected at hadron colliders, such as the LHC. The idea of the existence of the mirror symmetry in the Standard Model proposed by Lee-Yang would be a great starting point [12]. They proposed the existence of the heavy particles with exactly opposite chirality of the SM particles. However, these models were under the tight constraints of the precision measurements of the Standard Model, particularly the $S$ parameter.

One deeper motivation to build a model containing additional mirror fermions is to study the electroweak phase transition which is non-perturbative. The most popular framework to study non-perturbative phenomena is through lattice regularization. To put a chiral gauge theory such the Standard Model on the lattice, it is required to have an equal numbers of left-handed and right-handed fermions. As shown in chapter 1 , the Standard Model does not have this feature. Nevertheless, Montvay has shown that a gauge-invariant formulation of the Standard Model on the lattice is possible if one introduces mirror fermions [13]

Recently, the extension of the SM in the mirror symmetric direction has been renovated by the idea of extending the Higgs sector to avoid the conflict with the electroweak precision measurements. This is the model of Right-handed Neutrinos at Electroweak scale, in short EW $\nu_{R}$ proposed by Professor Pham Quang Hung in 2007 [14]. 


\subsection{The Model}

In this section, we describe the construction of the EW $\nu_{R}$ model [14].

\subsubsection{The Fermion Sector}

The original purpose of building the model is to generate the mass of neutrinos. So let start out with the mass term of the neutrino in one generation. In the Standard Model, the right-handed neutrinos are sterile singlets under the SM $S U(2)$ group. In the $\mathrm{EW} \nu_{R}$ model, we assume the existence of right-handed charged leptons $e_{R}^{M}$ with the opposite chirality to the SM charge leptons, then we can group the right-handed neutrinos into $S U(2)$ doublets. For one family, we have

$$
l_{R}^{M}=\left(\begin{array}{c}
\nu_{R} \\
e_{R}^{M}
\end{array}\right)
$$

The subscript $M$ stands for mirror fermions. In this case, we have a mirror lepton $S U(2)$ doublet exists along with the SM $S U(2)$ doublet. Therefore, we will name the gauge group of weak interactions as $S U(2)_{W}$ for better suitable representation. Using the same logic, we also assume the existence of a charged left-handed $S U(2)_{W}$ singlet mirror lepton $e_{L}^{M}$ as the mirror counterpart of the singlet $e_{R}$.

It must be emphasized that the mirror particles in the $\mathrm{EW} \nu_{R}$ model are completely different than the mirror objects in the other models, because the mirror particles in this model have the same quantum numbers as the SM particles except the opposite chirality.

With the new mirror lepton doublet, the Dirac mass of neutrinos can be extracted from the bilinear $\bar{l}_{L} l_{R}^{M}$, which is $2 \times 2=3+1$ in terms of the group structure. Thus one needs to introduce a singlet scalar field $\phi_{S}$ which can couple to the bilinear. In addition, the $\mathrm{EW} \nu_{R}$ model still contains the SM Higgs dou- 
blet $\Phi$. The lepton mass terms in the model can be realized through the Yukawa interactions as

$$
\begin{aligned}
\mathcal{L}_{S} & =g_{S l} \bar{l}_{L} \phi_{S} l_{R}^{M}+\text { h.c. } \\
& =g_{S l}\left(\bar{\nu}_{L} \nu_{R}+\bar{e}_{L} e_{R}^{M}\right) \phi_{S}+\text { h.c. } \\
\mathcal{L}_{Y 1} & =g_{l} \bar{l}_{L} \Phi e_{R}+\text { h.c. } \\
\mathcal{L}_{Y 2} & =g_{l}^{M} \bar{l}_{R}^{M} \Phi e_{L}^{M}+\text { h.c. }
\end{aligned}
$$

Once $\phi_{S}, \Phi$ develop their VEVs

$$
\begin{aligned}
& <\phi_{S}>=v_{S}, \\
& <\Phi>=\left(\begin{array}{c}
0 \\
\frac{v_{2}}{\sqrt{2}}
\end{array}\right)
\end{aligned}
$$

Then from Eqs.(3.2.2, 3.2.3), the mass of the Dirac neutrino and leptons are

$$
m_{\nu}^{D}=g_{S L} v_{S}, m_{l}=g_{l} v_{2} / \sqrt{2}, m_{l^{M}}=g_{l}^{M} v_{2} / \sqrt{2}
$$

The mass matrix for the charged SM and mirror leptons is

$$
M_{l}=\left(\begin{array}{cc}
m_{l} & m_{\nu}^{D} \\
m_{\nu}^{D} & m_{l^{M}}
\end{array}\right) .
$$

After diagonalizing this mass matrix, the eigenvalues corresponding to the masses of the charged lepton and its mirror counterpart are given by

$$
\begin{aligned}
\widetilde{m}_{l} & =m_{l}-\frac{\left(m_{\nu}^{D}\right)^{2}}{m_{l^{M}}-m_{l}}, \\
\widetilde{m}_{l^{M}} & =m_{l^{M}}+\frac{\left(m_{\nu}^{D}\right)^{2}}{m_{l^{M}}-m_{l}} .
\end{aligned}
$$

Assume that $m_{l^{M}} \gg m_{l}$, and the Dirac mass of neutrino $m_{\nu}^{D} \ll m_{l^{M}}$, then the mass mixing in Eq. (3.2.5) is negligible. We can have $\widetilde{m}_{l} \approx m_{l}, \widetilde{m}_{l^{M}} \approx m_{l^{M}}$. 
The Dirac mass of neutrino just depends on the coupling $g_{S L}$ and its VEV $v_{S}$. From the $\mu 2 e$ conversion work, $g_{S L}$ should be less than $10^{-3}[15]$. While the $v_{S}$ can be very small, because it is basically unconstrained by experiments. Therefore, one can make $m_{\nu}^{D}$ to be at the oder of $e V$.

As seen in the last chapter, neutrinos can have a Majorana mass due to their neutral charge property. In this model, the bilinear $l_{R}^{M, T} \sigma_{2} l_{R}^{M}$ will generate the Majorana mass of neutrinos. Under the group $S U(2)_{W} \times U(1)_{Y}$, the bilinear transforms as $(1+3, Y / 2=-1)$. We only can use a +1 charged scalar triplet to couple to the bilinear because the charge scalar singlet would break the charge conservation. One assumes the the existence of a scalar triplet $\vec{\chi}(3, Y / 2=+1)=$ $\left(\chi^{0}, \chi^{+}, \chi^{++}\right)$in an adjoint representation

$$
\tilde{\chi}=\frac{1}{\sqrt{2}} \vec{\tau} \vec{\chi}=\left(\begin{array}{cc}
\frac{1}{\sqrt{2}} \chi^{+} & \chi^{++} \\
\chi^{0} & -\frac{1}{\sqrt{2}} \chi^{+}
\end{array}\right)
$$

With the proper VEV:

$$
<\tilde{\chi}>=\left(\begin{array}{cc}
0 & 0 \\
v_{M} & 0
\end{array}\right)
$$

We can have a gauge invariant Yukawa coupling in the form of

$$
\mathcal{L}_{M}=g_{M} l_{R}^{M, T} \sigma_{2} \tau_{2} \widetilde{\chi} l_{R}^{M}
$$

After the scalar triplet gets VEV, the right-handed neutrino acquires a Majorana mass

$$
M_{R}=g_{M} v_{M}
$$

If the right-handed neutrino is lighter than $M_{Z} / 2$, then the $Z$ boson could decay into two right-handed neutrinos that contribute to the value of the width of the $Z$ boson. The precise measurement of Z-width puts a strict constraint on the number 
of light neutrinos to be three flavors of the SM neutrinos. In fact, in this model, we impose $M_{R}>M_{Z} / 2$, so that the right-handed neutrinos cannot contribute to the Z-width.

To prevent the left-handed neutrino from acquiring a Majorana mass, we impose a global symmetry $U(1)_{M}$ under which

$$
\begin{gathered}
l_{R}^{M}, e_{L}^{M} \longrightarrow e^{i \theta_{M}} l_{R}^{M}, e_{L}^{M}, \\
\tilde{\chi} \longrightarrow e^{-2 i \theta_{M}} \widetilde{\chi} \\
\phi_{S} \longrightarrow e^{-i \theta_{M}} \phi_{S} .
\end{gathered}
$$

All other fields are singlets under this $U(1)_{M}$ group. Consequently, the mass terms such as $g_{L} l_{L}^{T} \sigma_{2} \tau_{2} \widetilde{\chi} l_{L}, \bar{l}_{L} l_{R}^{M} \tilde{\chi}$ are forbidden. The left-handed neutrino does not have Majorana mass at tree level. However, it happens at the loop level:

$$
M_{L}=\lambda \frac{1}{16 \pi^{2}} \frac{m_{\nu}^{D 2}}{M_{R}} \ln \frac{M_{R}}{M_{\phi_{S}}} .
$$

Here $\lambda$ is the quartic coupling of $\phi_{S} ; M_{\phi_{S}}$ is the mass of $\phi_{S}$. The Majorana mass matrix is

$$
M_{M}=\left(\begin{array}{cc}
M_{L} & m_{\nu}^{D} \\
m_{\nu}^{D} & M_{R}
\end{array}\right)
$$

Here $m_{\nu}^{D}, M_{R}, M_{L}$ are given in Eq. (3.2.4, 3.2.10, 3.2.12), respectively. Diagonalizing the Majorana mass matrix give us two eigenvalues:

$$
\begin{aligned}
& m_{\nu}=M_{L}-\frac{\left(m_{\nu}^{D}\right)^{2}}{M_{R}} \approx-\frac{g_{S l}^{2}}{g_{M}} \frac{v_{S}^{2}}{v_{M}}(1-\epsilon), \\
& m_{N}=M_{R},
\end{aligned}
$$

with $\epsilon<10^{-2}$. Knowing that $v_{M}$ is at order of electroweak scale $\Lambda_{E W}$, the requirement of the mass of the light neutrino $m_{\nu}<2.3 \mathrm{eV}[6]$ is equivalent with $v_{S} \approx O\left(10^{5} \mathrm{eV}\right)$. In the EW $\nu_{R}$ model, the seesaw happens between two scales 
$k e V$ and $\Lambda_{E W}$. This is the crucial difference between this model with other models based on the seesaw mechanism which need to a very high scales at least at the order of $O\left(10^{14} \mathrm{GeV}\right)$.

In the quark sector, the anomaly cancellation requires the introduction of mirror quarks as well. For each family,

- $S U(2)_{W}$ quark doublets:

$$
S M: q_{L}=\left(\begin{array}{c}
u_{L} \\
d_{L}
\end{array}\right)\left(2, \frac{1}{3}\right) ; \text { Mirror }: q_{R}^{M}=\left(\begin{array}{c}
u_{R}^{M} \\
d_{R}^{M}
\end{array}\right)\left(2, \frac{1}{3}\right)
$$

for the SM left-handed quark doublet and for the right-handed mirror quark doublet respectively.

- $S U(2)_{W}$ quark singlets:

$$
S M: u_{R}\left(1, \frac{4}{3}\right), d_{R}\left(1,-\frac{2}{3}\right) ; \text { Mirror }: u_{L}^{M}\left(1, \frac{4}{3}\right), d_{L}^{M}\left(1,-\frac{2}{3}\right)
$$

for the right-handed SM quark singlets and left-handed mirror quark singlets respectively.

In the $\mathrm{EW} \nu_{R}$ model, the anomaly cancellation happens within the quark and lepton sectors themselves. We also have the masses of the quarks and the mirror quarks:

$$
\begin{aligned}
\widetilde{m}_{q} & =m_{q}-\frac{\left(m_{\mu}^{D}\right)^{2}}{m_{q^{M}}-m_{q}} \frac{g_{S q}}{g_{S l}} \approx m_{q}, \\
\widetilde{m}_{q^{M}} & =m_{q^{M}}+\frac{\left(m_{\mu}^{D}\right)^{2}}{m_{q^{M}}-m_{q}} \frac{g_{S q}}{g_{S l}} \approx m_{q^{M}},
\end{aligned}
$$

with $m_{q}=g_{q} v_{2} / \sqrt{2}, m_{q^{M}}=g_{q}^{M} v_{2} / \sqrt{2}$.

\subsubsection{The Scalar Sector}

So far, in the $\mathrm{EW} \nu_{R}$ model, we need at least one complex scalar triplet $\vec{\chi}$ as shown in Eq. (3.2.7) and a singlet $\phi_{S}$ to generate the mass of the light neutrino 
together with the complex doublet $\Phi$ of the Standard Model. It is well-known that the scalar triplet can break custodial symmetry at tree level explicitly. Generally, the contribution of a new Higgs representation to the $\rho$ parameter is given by

$$
\rho=\frac{\sum_{i}\left[T(T+1)-T_{3}^{2}\right]_{i} v_{i}^{2} c_{T, Y}}{2 \sum_{i} T_{3 i}^{2} v_{i}^{2}}
$$

where $c_{T, Y}=1$ for a complex multiplet and $c_{T, Y}=1 / 2$ for a real multiplet [16]. Any complex doublet contributes an amount of 1 to the $\rho$ parameter. While a triplet contribute an amount $1 / 2$. The $\rho$ parameter of a mixture of a triplet and a doublet would be different to 1 , and depends on the ratio of VEVs of the triplet and the doublet. $\rho$ can be closed to 1 if the VEV of the triplet is very small compare to that of the doublet. However, in the case of the $\mathrm{EW} \nu_{R}$ model, we expect the VEV of the triplet is at the order of $\Lambda_{E W}$. It necessitates the introduction of a real scalar triplet $\xi(3, Y / 2=0)$ with a proper choice of VEV so that one can ensure the $\rho=1$ constraint to be valid. We will show in details in the next few parts. The two triplets $\xi, \vec{\chi}$ form a $(3,3)$ representation under the global $S U(2)_{L} \times S U(2)_{R}$ symmetry as follows $[17,18,19,20]$

$$
\chi=\left(\begin{array}{ccc}
\chi^{0} & \xi^{+} & \chi^{++} \\
\chi^{-} & \xi^{0} & \chi^{+} \\
\chi^{--} & \xi^{-} & \chi^{0 *}
\end{array}\right)
$$

Similarly, $\phi$ and its adjoint $\tilde{\phi}=\imath \tau_{2} \phi^{*}$ can be grouped into a $(2,2)$ representation

$$
\Phi=\left(\begin{array}{cc}
\phi^{0 *} & \phi^{+} \\
\phi^{-} & \phi^{0}
\end{array}\right) \text {. }
$$

The kinetic part of the Lagrangian for the Higgs sector is

$$
\mathcal{L}_{K H}=\frac{1}{2} \operatorname{Tr}\left[\left(D_{\mu} \Phi\right)^{\dagger}\left(D^{\mu} \Phi\right)\right]+\frac{1}{2} \operatorname{Tr}\left[\left(D_{\mu} \chi\right)^{\dagger}\left(D^{\mu} \chi\right)\right]+\left|\partial_{\mu} \phi_{S}\right|^{2} .
$$


Here, the derivatives of the scalars are expressed as

$$
\begin{aligned}
& D_{\mu} \Phi=\partial_{\mu} \Phi+i g \vec{W} \frac{\vec{\tau}}{2} \Phi-i g^{\prime} \Phi B \frac{\tau_{3}}{2} \\
& D_{\mu} \chi=\partial_{\mu} \chi+i g \vec{W} \frac{\vec{t}}{2} \chi-i g^{\prime} \chi B \frac{t_{3}}{2}
\end{aligned}
$$

The most general scalar potential for $\Phi$ and $\chi$ that preserves global $S U(2)_{L} \times$ $S U(2)_{R}$ is given by [17, 20]:

$$
\begin{aligned}
V(\Phi, \chi) & =\lambda_{1}\left(\operatorname{Tr} \Phi^{\dagger} \Phi-v_{2}^{2}\right)^{2}+\lambda_{2}\left(\operatorname{Tr} \chi^{\dagger} \chi-3 v_{M}^{2}\right)^{2} \\
& +\lambda_{3}\left(\operatorname{Tr} \Phi^{\dagger} \Phi-v_{2}^{2}+\operatorname{Tr} \chi^{\dagger} \chi-3 v_{M}^{2}\right)^{2} \\
& +\lambda_{4}\left(\left(\operatorname{Tr} \Phi^{\dagger} \Phi\right)\left(\operatorname{Tr} \chi^{\dagger} \chi\right)-2\left(\operatorname{Tr} \Phi^{\dagger} \frac{\tau^{a}}{2} \Phi \frac{\tau^{b}}{2}\right)\left(\operatorname{Tr} \chi^{\dagger} T^{a} \chi T^{b}\right)\right) \\
& +\lambda_{5}\left(3 \operatorname{Tr} \chi^{\dagger} \chi \chi^{\dagger} \chi-\left(\operatorname{Tr} \chi^{\dagger} \chi\right)^{2}\right)
\end{aligned}
$$

Here, $\lambda_{i}$ are the coupling constants of the self-coupling of the Higgs fields. In order to have a positive semi-definite potential, the following conditions of these coupling constants must be satisfied:

$$
\left\{\begin{array}{l}
\lambda_{1}+\lambda_{2}+2 \lambda_{3}>0 \\
\lambda_{1} \lambda_{2}+\lambda_{1} \lambda_{3}+\lambda_{2} \lambda_{3}>0 \\
\lambda_{4}>0, \quad \lambda_{5}>0
\end{array}\right.
$$

\subsubsection{The SSB of the $\mathrm{EW} \nu_{R}$ Model}

A proper choice of the VEVs of $\chi, \Phi$ is given by

$$
\langle\chi\rangle=\left(\begin{array}{ccc}
v_{M} & 0 & 0 \\
0 & v_{M} & 0 \\
0 & 0 & v_{M}
\end{array}\right),
$$

and

$$
\langle\Phi\rangle=\left(\begin{array}{cc}
v_{2} / \sqrt{2} & 0 \\
0 & v_{2} / \sqrt{2}
\end{array}\right)
$$


When $\chi, \Phi$ develop their VEVs, then the global symmetry $S U(2)_{L} \times S U(2)_{R} \longrightarrow$ $S U(2)_{C}$. At the same time, the local gauge group of the electroweak interactions is broken spontaneously as $S U(2)_{W} \times U(1)_{Y} \longrightarrow U(1)_{e m}$. The gauge vector bosons $W / Z$ acquire their masses from the longitudinal components of the N-G bosons. One obtains

$$
\begin{aligned}
& M_{W}=g v / 2, \\
& M_{Z}=M_{W} / \cos \theta_{W},
\end{aligned}
$$

with $v=\sqrt{v_{2}^{2}+8 v_{M}^{2}} \approx 246 \mathrm{GeV}$ and, at tree level:

$$
\rho=\frac{M_{W}}{M_{Z} \cos \theta_{W}}=1 .
$$

The custodial symmetry is preserved at tree level.

One crucial point is to find the ranges of the VEVs, $v_{2}, v_{M}$, of the scalar doublet and triplets in the $\mathrm{EW} \nu_{R}$ model. They are obviously bounded by the value of $v=246 \mathrm{GeV}$. Moreover, $v_{M}$ appears on the mass of the right-handed neutrino $M_{R}=g_{M} v_{M}$, and in the Yukawa coupling constants. We will have some constraints on the ranges of $v_{M}, v_{2}$ in the next chapter.

\subsubsection{The Mass Spectrum of the Scalars}

After the spontaneous breaking of $S U(2)_{L} \times U(1)_{Y}$, besides the three NambuGoldstone bosons which are absorbed by $\mathrm{W}$ and $\mathrm{Z}$, there are ten physical scalars grouped into the multiplets of the group $S U(2)_{D}$. To present these fields, we introduce subsidiary fields

$$
\begin{aligned}
\phi^{0} & \equiv \frac{1}{\sqrt{2}}\left(v_{2}+\phi^{0 r}+i \phi^{0 i}\right) \\
\chi^{0} & \equiv v_{M}+\frac{1}{\sqrt{2}}\left(\chi^{0 r}+i \chi^{0 i}\right) \\
\psi^{ \pm} & \equiv \frac{1}{\sqrt{2}}\left(\chi^{ \pm}+\xi^{ \pm}\right) \\
\zeta^{ \pm} & \equiv \frac{1}{\sqrt{2}}\left(\chi^{ \pm}-\xi^{ \pm}\right) .
\end{aligned}
$$


The first two fields are the mixing of the neutral components of the complex scalar triplet $\vec{\chi}$ and the scalar doublet $\Phi$. While the last ones are the mixing of the charged components of two triplet scalars. There is also the mixing angle between the doublet and the triplet:

$$
\begin{aligned}
\sin \theta_{H} & \equiv s_{H}=\frac{2 \sqrt{2} v_{M}}{v}, \\
\cos \theta_{H} & \equiv c_{H}=\frac{v_{2}}{v}
\end{aligned}
$$

We have three N-G bosons expressed as:

$$
\begin{aligned}
G_{3}^{ \pm} & =c_{H} \phi^{ \pm}+s_{H} \psi^{ \pm} \\
G_{3}^{0} & =i\left(-c_{H} \phi^{0 i}+s_{H} \chi^{0 i}\right) .
\end{aligned}
$$

In the scalar sector, we have 13 degrees of freedom from one complex scalar doublet, one complex scalar triplet and one real scalar triplet. Three of them are the N-G bosons listed above. The remaining ten degrees of freedom correspond to ten physical Higgs fields. Under the custodial group $S U(2)_{C}$, they are classified into:

- A fivetet: $H_{5}^{ \pm \pm}, H_{5}^{ \pm}, H_{5}^{0}$

- A triplet: $H_{3}^{ \pm}, H_{3}^{0}$

- Two singlets: $H_{1}^{0}, H_{1}^{0 \prime}$.

Where,

$$
\begin{aligned}
H_{5}^{++} & =\chi^{++}, H_{5}^{+}=\zeta^{+}, H_{3}^{+}=c_{H} \psi^{+}-s_{H} \phi^{+} \\
H_{5}^{0} & =\frac{1}{\sqrt{6}}\left(2 \xi^{0}-\sqrt{2} \chi^{0 r}\right), H_{3}^{0}=i\left(c_{H} \chi^{0 i}+s_{H} \phi^{0 i}\right), \\
H_{1}^{0} & =\phi^{0 r}, H_{1}^{0 \prime}=\frac{1}{\sqrt{3}}\left(\sqrt{2} \chi^{0 r}+\xi^{0}\right)
\end{aligned}
$$

with $H_{5}^{--}=\left(H_{5}^{++}\right)^{*}, H_{5}^{-}=-\left(H_{5}^{+}\right)^{*}, H_{3}^{-}=-\left(H_{3}^{+}\right)^{*}$, and $H_{3}^{0}=-\left(H_{3}^{0}\right)^{*}$. 
As discussed in [17], the potential in Eq. (5.3.12) breaks the group $U(1)_{M}$ explicitly due to the $\lambda_{4}$ interaction. Therefore the model does not have the NambuGoldston boson associated with this symmetry breaking.

\subsection{Summary of The EW $\nu_{R}$ Model}

- With the extension in both the lepton and quark sectors, the model is anomaly free within these sectors. The mirror particles contribute to the triangle loops at the same amount but in the opposite sign with the contribution of the SM counterparts. Eventually, the triangle loops are trivial among the leptons and quarks individually.

- The biggest difference between the $\mathrm{EW} \nu_{R}$ model in comparison with other seesaw models is the upper bound in the energy scale. By introducing the scalar singlet $\phi_{S}$, the lower bound only depends on the coupling and the VEV of the singlet which are basically not tightly constrained. We then have the seesaw of the $k e V$ and $M e V$ scale as seen in Eq. (3.2.14). The upper limit of the $\mathrm{EW} \nu_{R}$ model is bounded by the electroweak scale $\Lambda_{E W}$.

- Though it is not the priority of building the model, the $\mathrm{EW} \nu_{R}$ model can be merged into the higher symmetric group $E_{6}$ of a unified theory. In these representations, the SM fermions and their mirror counterparts belong to $\mathbf{2 7} \mathbf{7}_{L}$ and $\mathbf{2 7 _ { L } ^ { c }}$ of $E_{6}$, respectively. The Dirac mass term in Eq. (3.2.4) is extracted from the term $2 \boldsymbol{7}_{L}^{c, T} \sigma_{2} \mathbf{2} \mathbf{7}_{L} \phi_{S}(1)$, where $\phi_{S}(1)$ is an $E_{6}$ singlet. The Majorana mass term in Eq. (3.2.10) comes from the term $\mathbf{2 7}_{L}^{T} \sigma_{2} \mathbf{2} \mathbf{7}_{L}$. More details can be found in [21].

- The biggest advantage of the $\mathrm{EW} \nu_{R}$ model is the accessibility at the current and near future high energy colliders, such as the LHC and the ILC. The right-handed neutrinos in the model belong to the $S U(2)_{W}$ doublets, so they 
couple to the gauge bosons directly. As the result, their production crosssection and decay rate are at the electroweak order.

- In terms of the cosmological consequence, one might suspect the contribution of the right-handed neutrinos to the total energy density. However, in the EW $\nu_{R}$ model, $\nu_{R} \longrightarrow \nu_{L}+\phi_{S} . \nu_{L}$ is the remnant of these processes, and only its mass contributes to the total energy density. Moreover, the nucleosynthesis is supposed to happen at $T \approx M e V$. By that time, all right-handed neutrinos would have decayed into $\nu_{L}$. The number of the light neutrinos is still limited to 3 families of the SM neutrinos. Thus the right-handed neutrinos do not affect the big bang nucleosynthesis. 


\section{Chapter 4}

\section{Electroweak Precision Measurements}

Over a couple of decades, experimental results in High Energy Physics have been making a great progress on the electroweak precision measurements of the Standard Model. Especially the $Z$ pole, the $W$ mass, and low energy data can be used to search for and set limits on any deviation from the SM. Satisfying the electroweak precision measurements is a crucial test of any BSM model. We devote this chapter to investigate the $\mathrm{EW} \nu_{R}$ model under these constraints.

\subsection{Oblique Parameters}

Among several ways to constrain the effect of New Physics on the Standard Model, the oblique parameters, $S, T, U$, are the most common set of observables $[22,23]$. By definition, $S, T, U$ contain the electroweak contributions of the new particles into the self-energy 2-point function of the SM vector bosons at the loop levels.

- S measures the momentum dependence of vacuum polarization.

$$
\alpha S \equiv 4 e^{2}\left[\Pi_{33}^{\prime}(0)-\Pi_{3 Q}^{\prime}(0)\right]
$$


- T measures the custodial isospin symmetry violation.

$$
\alpha T \equiv \frac{e^{2}}{s_{W}^{2} c_{W}^{2} M_{Z}^{2}}\left[\Pi_{11}(0)-\Pi_{33}(0)\right]
$$

$T$ parameter relates to the custodial $\rho$ parameter, which is defined as

$$
\rho \approx 1+\alpha T
$$

- $U$ parameter is less important than $S$ and $T$ in constraining New Physics, and is usually small compared to $S$ and $T$. In terms of the self-energy 2-point functions,

$$
\alpha T \equiv 4 e^{2}\left[\Pi_{11}^{\prime}(0)-\Pi_{33}^{\prime}(0)\right]
$$

In these definitions, $s_{W}=\sin \theta_{W}, c_{W}=\cos \theta_{W} ; \theta_{W}$ is the weak mixing angle. The functions $\Pi_{11}, \Pi_{33}$ are the vacuum polarizations of the isospin currents, and $\Pi_{3 Q}$ is the vacuum polarization of the third isospin and the electromagnetic current. While the $\Pi^{\prime}$ is defined as

$$
\Pi^{\prime}(0) \equiv \frac{\Pi\left(q^{2}\right)-\Pi(0)}{q^{2}}
$$

Generally, these functions are evaluated at $q^{2}=M_{Z}^{2}$. We just calculate the contribution of New Physics (NP) in the EW $\nu_{R}$ model on $S$ and $T$, because $U$ is generally much smaller than the two. In terms of the self-energy of the $W, Z$ and $\gamma$ bosons and the $Z \gamma$ mixing [22], $S$ and $T$ can be expressed as

$$
\begin{aligned}
\frac{\hat{\alpha}\left(M_{Z}\right)}{4 \hat{s}_{W}^{2} \hat{c}_{W}^{2}} S \equiv & \frac{\Pi_{Z Z}\left(M_{Z}^{2}\right)-\Pi_{Z Z}(0)}{M_{Z}^{2}}- \\
& \frac{\hat{c}_{W}^{2}-\hat{s}_{W}^{2}}{\hat{c}_{W} \hat{s}_{W}} \frac{\Pi_{Z \gamma}\left(M_{Z}^{2}\right)}{M_{Z}^{2}}-\frac{\Pi_{\gamma \gamma}\left(M_{Z}^{2}\right)}{M_{Z}^{2}} \\
\hat{\alpha}\left(M_{Z}\right) T \equiv & \frac{\Pi_{W W}(0)}{M_{W}^{2}}-\frac{\Pi_{Z Z}(0)}{M_{Z}^{2}}
\end{aligned}
$$

Note that the running constants, $\hat{\alpha}, \hat{s}_{W}, \hat{c}_{W}$ are evaluated at $q^{2}=M_{Z}^{2}$ in the minimal subtraction scheme $(\overline{M S})$ of the renormalization procedure. Moreover, 
at one loop level, these parameters can be decomposed into the contributions of New Physics (NP) and of the Standard Model. In the framework of the EW $\nu_{R}$ model, we can present the effect of the NP in terms of $\widetilde{S}, \widetilde{T}$ which are defined as

$$
\begin{aligned}
& \widetilde{S}=S^{E W \nu_{R}}-S^{S M} \\
& \widetilde{T}=T^{E W \nu_{R}}-T^{S M}
\end{aligned}
$$

We consider the contributions of the scalars and the fermions individually. Therefore we separate $\widetilde{S}, \widetilde{T}$ into

$$
\begin{aligned}
\widetilde{S} & =\widetilde{S}_{\text {scalar }}+\widetilde{S}_{\text {fermion }} \\
\widetilde{T} & =\widetilde{T}_{\text {scalar }}+\widetilde{T}_{\text {fermion }}
\end{aligned}
$$

The contributions of NP of the $\mathrm{EW} \nu_{R}$ model in the scalar and the fermion sectors can be expressed explicitly by

$$
\begin{aligned}
\widetilde{S}_{\text {scalar }} & =S_{\text {scalar }}^{E W \nu_{R}}-S_{\text {scalar }}^{S M} \\
\widetilde{S}_{\text {fermion }} & =S_{\text {fermion }}^{E W \nu_{R}}-S_{\text {fermion }}^{S M} \\
\widetilde{T}_{\text {scalar }} & =T_{\text {scalar }}^{E W \nu_{R}}-T_{\text {scalar }}^{S M} \\
\widetilde{T}_{\text {fermion }} & =T_{\text {fermion }}^{E W \nu_{R}}-T_{\text {fermion }}^{S M}
\end{aligned}
$$

\subsection{The Oblique Parameters of the $\mathrm{EW} \nu_{R}$ Model}

First, we will give an overview about the contributions of the new scalar and fermion sectors of the model to the oblique parameters separately. Generally, the introduction of a heavy chiral doublet gives a positive contribution to the $S$ parameter. Also, a heavy non-degenerate multiplet of fermions contributes positively to $T$. In the $\mathrm{EW} \nu_{R}$ model, the existence of the new mirror fermions and right-handed neutrinos at the electroweak scale could potentially ruin the constraint in the $S, T$ parameters. However, in this model, there also exists the scalar triplet which can provide a negative contribution to $S$ and $T$ in a certain 
parameter space. As pointed out in [24], there is a cancellation of the contributions of the new scalars and the new fermion in the $\mathrm{EW} \nu_{R}$ model to $S, T$, then these parameters remain in the allowed range as with the condition $U=0[23]^{1}$

$$
\begin{aligned}
& S=-0.02 \pm 0.14 \\
& T=0.06 \pm 0.14
\end{aligned}
$$

We will present the contributions of New Physics to the $S, T$ parameters at 1 loop level of the new scalars and fermions in the $\mathrm{EW} \nu_{R}$ model separately in the next section [25].

\subsection{The Contribution of Mirror Fermions to $S, T$}

In the $\mathrm{EW} \nu_{R}$ model, there exist six mirror quarks, three mirror charged leptons, and three right-handed neutrinos as listed in the section (3.2). Basically, we have six new chiral doublets from these particles. There are four types of diagrams contributing to $\widetilde{S}, \widetilde{T}$ as seen in Eq. (4.1.6).

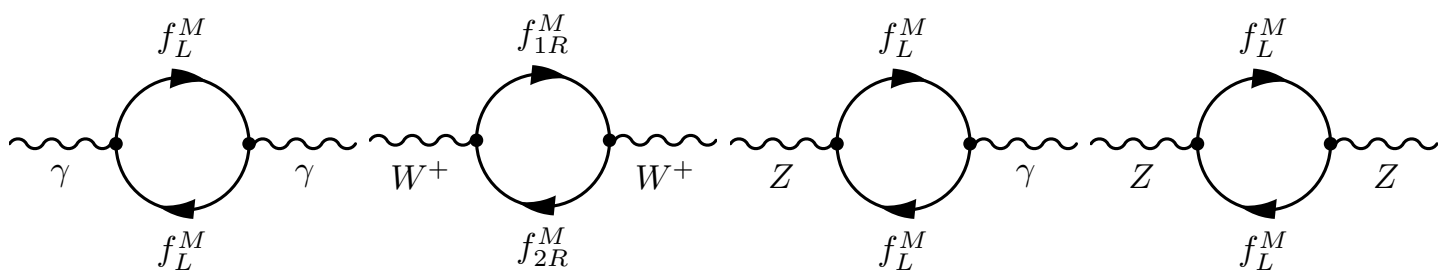

Figure 4.1. The fermionic contributions to the self-energy 2-point function of gauge vector bosons

We can decompose $\widetilde{S}_{\text {fermion }}, \widetilde{T}_{\text {fermion }}$ into the contributions of the mirror quarks and mirror leptons as

$$
\begin{aligned}
& \widetilde{S}_{\text {fermion }}=\widetilde{S}_{\text {lepton }}+\widetilde{S}_{\text {quark }} \\
& \widetilde{T}_{\text {fermion }}=\widetilde{T}_{\text {lepton }}+\widetilde{T}_{\text {quark }}
\end{aligned}
$$

\footnotetext{
${ }^{1}$ These constraints were from the data at the time when our analysis was conducted in 2013. In the most updated results, $S=0.00 \pm-0.08, T=0.05 \pm 0.07$.
} 
Using the definition in Eq. (4.1.7), we find the contribution of New Physics in the fermion sector in the $\mathrm{EW} \nu_{R}$ model to be

- $\widetilde{S}_{\text {lepton }}$,

$$
\begin{aligned}
\widetilde{S}_{\text {lepton }}= & S_{\text {lepton }}^{E W \nu_{R}}-S_{\text {lepton }}^{S M} \\
= & \frac{\left(N_{C}\right)_{\text {lepton }}}{6 \pi} \sum_{i=1}^{3}\left\{-2 Y_{\text {lepton }} x_{\nu i}+2\left(-4 \frac{Y_{\text {lepton }}}{2}+3\right) x_{e i}-\right. \\
& -Y_{\text {lepton }} \ln \left(\frac{x_{\nu i}}{x_{e i}}\right)+\left(1-x_{\nu i}\right) \frac{Y_{\text {lepton }}}{2} G\left(x_{\nu i}\right) \\
& \left.+\left[\left(\frac{3}{2}-\frac{Y_{\text {lepton }}}{2}\right) x_{\text {ei }}-\frac{Y_{\text {lepton }}}{2}\right] G\left(x_{e i}\right)\right\}
\end{aligned}
$$

- $\widetilde{T}_{\text {lepton }}$,

$$
\begin{aligned}
\widetilde{T}_{\text {lepton }}= & T_{\text {lepton }}^{E W \nu_{R}}-T_{\text {lepton }}^{S M} \\
= & \frac{\left(N_{C}\right)_{\text {lepton }}}{4 \pi s_{W}^{2} M_{W}^{2}} \sum_{i=1}^{3}\left[m_{\nu i}^{2}\left(B_{1}\left(0 ; m_{\nu i}^{2}, m_{\nu i}^{2}\right)-B_{1}\left(0 ; m_{\nu i}^{2}, m_{e i}^{2}\right)\right)\right. \\
& \left.+m_{e i}^{2}\left(B_{1}\left(0 ; m_{e i}^{2}, m_{e i}^{2}\right)-B_{1}\left(0 ; m_{e i}^{2}, m_{\nu i}^{2}\right)\right)\right] \\
= & \frac{\left(N_{C}\right)_{l e p t o n}}{8 \pi s_{W}^{2} M_{W}^{2}} \sum_{i=1}^{3} \mathcal{F}\left(m_{\nu i}^{2}, m_{e i}^{2}\right)
\end{aligned}
$$


- $\widetilde{S}_{\text {quark }}$,

$$
\begin{aligned}
\widetilde{S}_{\text {quark }}= & S_{\text {quark }}^{E W \nu_{R}}-S_{\text {quark }}^{S M} \\
= & \frac{\left(N_{C}\right)_{\text {quark }}}{6 \pi} \sum_{i=1}^{3}\left\{2\left(4 \frac{Y_{\text {quark }}}{2}+3\right) x_{u i}\right. \\
& +2\left(-4 \frac{Y_{\text {quark }}}{2}+3\right) x_{d i}-Y_{\text {quark }} \ln \left(\frac{x_{u i}}{x_{d i}}\right) \\
& +\left[\left(\frac{3}{2}+Y_{\text {quark }}\right) x_{u i}+\frac{Y_{\text {quark }}}{2}\right] G\left(x_{u i}\right) \\
& \left.+\left[\left(\frac{3}{2}-Y_{\text {quark }}\right) x_{d i}-\frac{Y_{\text {quark }}}{2}\right] G\left(x_{d i}\right)\right\}
\end{aligned}
$$

- $\widetilde{T}_{\text {quark }}$,

$$
\begin{aligned}
\widetilde{T}_{\text {quark }} & =T_{\text {quark }}^{E W \nu_{R}}-T_{\text {quark }}^{S M} \\
& =\frac{\left(N_{C}\right)_{q u a r k}}{4 \pi s_{W}^{2} M_{W}^{2}} \sum_{i=1}^{3}\left[m_{u i}^{2}\left(B_{1}\left(0 ; m_{u i}^{2}, m_{u i}^{2}\right)-B_{1}\left(0 ; m_{u i}^{2}, m_{d i}^{2}\right)\right)\right. \\
& \left.+m_{d i}^{2}\left(B_{1}\left(0 ; m_{d i}^{2}, m_{d i}^{2}\right)-B_{1}\left(0 ; m_{d i}^{2}, m_{u i}^{2}\right)\right)\right] \\
& =\frac{\left(N_{C}\right)_{q u a r k}}{8 \pi s_{W}^{2} M_{W}^{2}} \sum_{i=1}^{3} \mathcal{F}\left(m_{u i}^{2}, m_{d i}^{2}\right),
\end{aligned}
$$

Here, all the $B_{1}\left(q^{2}, m_{1}^{2}, m_{2}^{2}\right), F\left(m_{1}^{2}, m_{2}^{2}\right), G(x)$ functions are written explicitly in Appendix (A.2). The summation here is taken over three families of the mirror fermions. The color number $\left(N_{C}\right)_{\text {quark }}=3$ for three colors of the mirror quarks, $\left(N_{C}\right)_{\text {lepton }}=1$ for the mirror leptons. For the hypercharge, $Y_{\text {lepton }}=-1$ for the mirror leptons, and $Y_{\text {quark }}=-1 / 3$ for the mirror quarks. The scalar variable $x_{u_{i}, d_{i}, \nu_{i}, e_{i}}=\left(m_{u_{i}, d_{i}, \nu_{i}, e_{i}} / M_{Z}\right)^{2}$.

In this thesis, we have not considered the mixing between the mirror fermions. We can justify this argument because all the mirror quarks and mirror leptons in 
the $\mathrm{EW} \nu_{R}$ model are at the order of $\Lambda_{E W}$, few hundred $G e V$. Eventually, their masses are relatively close to each other. The mixing matrices, therefore, would be very similar to the diagonal matrix among both the mirror quarks and the mirror leptons. The effect of mixing between generations is not important in the context of the electroweak precision measurements.

\subsection{The Contribution of the Scalars to $S, T$}

In the $\mathrm{EW} \nu_{R}$ model, the mass spectrum in the scalar sector consists of a scalar quintet, $H_{5}^{ \pm \pm}, H_{5}^{ \pm}, H_{5}^{0}$, a scalar triplet, $H_{3}^{ \pm}, H_{3}^{0}$, and two scalar singlets, $H_{1}^{0}, H_{1}^{0 \prime}$ under the custodial group $S U(2)_{D}$. In Feynman gauge, the NambuGoldstone bosons $G_{3}^{ \pm}, G_{3}^{0}$ also contribute to the oblique parameters. All of these new particles appear in the scalar loops of the self-energy diagram of the gauge bosons. For the case of the photon, the Feynman diagrams are

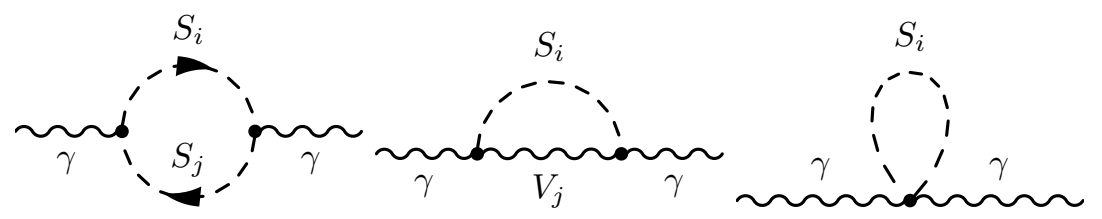

Figure 4.2. The contribution to the self-energy diagrams of the gauge bosons

Here $S_{i}$ presents the scalars and N-G bosons of the EW $\nu_{R}$ model. There are the same kind of diagrams for $W, Z$ and the mixing $Z-\gamma$. In terms of one-point and two-point functions, the contribution of New Physics of the scalar sector of the $\mathrm{EW} \nu_{R}$ model to the oblique parameters can be expressed as

- For $\widetilde{S}$ : 


$$
\begin{aligned}
& \widetilde{S}_{\text {scalar }}= S_{\text {scalar }}^{E W \nu_{R}}-S_{\text {scalar }}^{S M} \\
&= \frac{1}{M_{Z}^{2} \pi}\left\{\frac{4}{3} s_{H}^{2}\left[\bar{B}_{22}\left(M_{Z}^{2} ; M_{Z}^{2}, m_{H_{5}^{0}}^{2}\right)-M_{Z}^{2} \bar{B}_{0}\left(M_{Z}^{2} ; M_{Z}^{2}, m_{H_{5}^{0}}^{2}\right)\right]\right. \\
&+2 s_{H}^{2}\left[\bar{B}_{22}\left(M_{Z}^{2} ; M_{Z}^{2}, m_{H_{5}^{+}}^{2}\right)-M_{W}^{2} \bar{B}_{0}\left(M_{Z}^{2} ; M_{Z}^{2}, m_{H_{5}^{+}}^{2}\right)\right] \\
&+c_{H}^{2}\left[\bar{B}_{22}\left(M_{Z}^{2} ; M_{Z}^{2}, m_{H_{1}}^{2}\right)-M_{Z}^{2} \bar{B}_{0}\left(M_{Z}^{2} ; M_{Z}^{2}, m_{H_{1}}^{2}\right)\right] \\
&+\frac{8}{3} s_{H}^{2}\left[\bar{B}_{22}\left(M_{Z}^{2} ; M_{Z}^{2}, m_{H_{1}^{\prime}}^{2}\right)-M_{Z}^{2} \bar{B}_{0}\left(M_{Z}^{2} ; M_{Z}^{2}, m_{H_{1}^{\prime}}^{2}\right)\right] \\
&+\frac{4}{3} c_{H}^{2} \bar{B}_{22}\left(M_{Z}^{2} ; m_{H_{5}^{0}}^{2}, m_{H_{3}^{0}}^{2}\right)+2 c_{H}^{2} \bar{B}_{22}\left(M_{Z}^{2} ; m_{H_{5}^{+}}^{2}, m_{H_{3}^{+}}^{2}\right) \\
&+s_{H}^{2} \bar{B}_{22}\left(M_{Z}^{2} ; m_{H_{3}^{0}}^{2}, m_{H_{1}}^{2}\right)+\frac{8}{3} c_{H}^{2} \bar{B}_{22}\left(M_{Z}^{2} ; m_{H_{3}^{0}}^{2}, m_{H_{1}^{\prime}}^{2}\right) \\
&-4 \bar{B}_{22}\left(M_{Z}^{2} ; m_{H_{5}^{++}}^{2}, m_{H_{5}^{++}}^{2}\right)-\bar{B}_{22}\left(M_{Z}^{2} ; m_{H_{5}^{+}}^{2}, m_{H_{5}^{+}}^{2}\right)-\bar{B}_{22}\left(M_{Z}^{2} ; m_{H_{3}^{+}}^{2}, m_{H_{3}^{+}}^{2}\right) \\
&\left.-\left[\bar{B}_{22}\left(M_{Z}^{2} ; M_{Z}^{2}, m_{H}^{2}\right)-M_{Z}^{2} \bar{B}_{0}\left(M_{Z}^{2} ; M_{Z}^{2}, m_{H}^{2}\right)\right]\right\} \\
&(4.4 .1) \\
& \\
&
\end{aligned}
$$


- For $\widetilde{T}$ :

$$
\begin{aligned}
\widetilde{T}_{\text {scalar }}= & T_{\text {scalar }}^{E W \nu_{R}}-T_{\text {scalar }}^{S M} \\
= & \frac{1}{4 \pi s_{W}^{2} M_{W}^{2}}\left\{\frac{1}{2} \mathcal{F}\left(m_{H_{5}^{++}}^{2}, m_{H_{5}^{0}}^{2}\right)+\frac{3}{4} \mathcal{F}\left(m_{H_{5}^{+}}^{2}, m_{H_{5}^{0}}^{2}\right)+\frac{1}{4} \mathcal{F}\left(m_{H_{3}^{+}}^{2}, m_{H_{3}^{0}}^{2}\right)\right. \\
& +\frac{c_{H}^{2}}{2} \mathcal{F}\left(m_{H_{5}^{++}}^{2}, m_{H_{3}^{+}}^{2}\right)+\frac{c_{H}^{2}}{4} \mathcal{F}\left(m_{H_{5}^{+}}^{2}, m_{H_{3}^{0}}^{2}\right)+\frac{c_{H}^{2}}{12} \mathcal{F}\left(m_{H_{5}^{0}}^{2}, m_{H_{3}^{+}}^{2}\right) \\
& -\frac{c_{H}^{2}}{2} \mathcal{F}\left(m_{H_{5}^{+}}^{2}, m_{H_{3}^{+}}^{2}\right)-\frac{c_{H}^{2}}{3} \mathcal{F}\left(m_{H_{5}^{0}}^{2}, m_{H_{3}^{0}}^{2}\right)-\frac{s_{H}^{2}}{4} \mathcal{F}\left(M_{W}^{2}, m_{H_{5}^{+}}^{2}\right) \\
& +\frac{s_{H}^{2}}{4}\left[\mathcal{F}\left(m_{H_{3}^{+}}^{2}, m_{H_{1}}^{2}\right)-\mathcal{F}\left(m_{H_{3}^{0}}^{2}, m_{H_{1}}^{2}\right)\right] \\
& +\frac{2}{3} c_{H}^{2}\left[\mathcal{F}\left(m_{H_{3}^{+}}^{2}, m_{H_{1}^{\prime}}^{2}\right)-\mathcal{F}\left(m_{H_{3}^{0}}^{2}, m_{H_{1}^{\prime}}^{2}\right)\right] \\
& +\frac{s_{H}^{2}}{2} \mathcal{F}\left(M_{W}^{2}, m_{H_{5}^{++}}^{2}\right)-\frac{s_{H}^{2}}{4} \mathcal{F}\left(M_{W}^{2}, m_{H_{5}^{0}}^{2}\right)+M_{W}^{2} s_{H}^{2} B_{0}\left(0 ; M_{W}^{2}, m_{H_{5}^{0}}^{2}\right) \\
& \left.+M_{W}^{2} s_{H}^{2} B_{0}\left(0 ; M_{W}^{2}, m_{H_{5}^{+}}^{2}\right)-M_{W}^{2} s_{H}^{2} B_{0}\left(0 ; M_{W}^{2}, m_{H_{5}^{++}}^{2}\right)\right\}
\end{aligned}
$$

Here, all the $B_{1}\left(q^{2}, m_{1}^{2}, m_{2}^{2}\right), F\left(m_{1}^{2}, m_{2}^{2}\right), G(x)$ functions are written explicitly in Appendix (A.2). In the next section, we will analyze numerically the contribution of New Physics in both the scalar and fermionic sectors of the $E W \nu_{R}$ model to the oblique parameters.

\subsection{Numerical Analysis}

In this section, we first present the contributions of New Physics of the EW $\nu_{R}$ model in the scalar and fermionic sectors separately to the oblique parameters in the whole parameter space. We investigate the dependence of $S$ and $T$ on the mass splittings in a mirror fermion family and in the scalar multiplets. Finally, we apply the electroweak precision measurements to the total $S$ and $T$ to find the allowed region of the model. 


\subsubsection{The Parameter Space}

In addition to the $\mathrm{SM}$ parameters, the $\mathrm{EW} \nu_{R}$ model contains twenty independent parameters including the masses of six mirror quarks, three mirror charged lepton, three right-handed neutrinos, seven scalars, and one mixing angle. In the scalar sector, we have a quintet $H_{5}^{0, \pm, \pm \pm}$, a triplet $H_{3}^{0, \pm}$, and two singlets $H_{1}^{0}, H_{1}^{0 \prime}$. For the purpose of finding the allowed region satisfying the electroweak precision measurements, we scan them from $M_{Z}$ to the unitary bound $\approx 650 \mathrm{GeV}$. It is the same for the scanned range of the masses of the mirror quarks and the mirror charged leptons, $M_{Z} \leq m_{\tilde{q}} \leq 650 \mathrm{GeV}$. The mixing angle $\theta_{H}$ presents the mixing between the doublet $\Phi$ and the triplet $\tilde{\chi}$ after the spontaneous symmetry breaking of the gauge group. As discussed in [20], $0.1 \leq \sin \theta_{H} \leq 0.89$.

For the right-handed neutrinos, their masses have the form of $M_{R}=g_{M} v_{M}$. Here $v_{M}$ is the VEV of the triplet scalar $\tilde{\chi}, g_{M}$ is the Yukawa coupling of the scalar triplet with the mirror fermions. While the mass of the Standard Model particles, such as the top quark, are produced through the VEV of the doublet $\Phi$, $v_{2}$, in the form of $m_{f}=g_{f} v_{2}$. To ensure that the model is a perturbative theory, we require the coupling constant to satisfy the constraint $\alpha_{f}=g_{f}^{2} /(4 \pi) \leq 1$. With the mass of top quark known to be $173 \mathrm{GeV}$, the perturbative requirement is equivalent to $v_{2} \geq 68 \mathrm{GeV}$. As we know, after spontaneous symmetry breaking, $v_{2}^{2}+8 v_{M}^{2}=v^{2}=(246 \mathrm{GeV})^{2}$. This means that $v_{M} \leq 84 \mathrm{GeV}$. To avoid conflict with the Z-width measurement, we require $M_{R}=g_{M} v_{M} \geq M_{Z} / 2$. Also the perturbative requirement gives $g_{M} \leq \sqrt{4 \pi}$. Combining the two requirements, we have $v_{M} \geq 13 \mathrm{GeV}, v_{2} \leq 243 \mathrm{GeV}$. Finally, we have the upper limit on the mass of right-handed neutrinos is $M_{R} \leq 300 \mathrm{GeV}$. Note that if we relax the perturbative condition $g_{M} \leq O(\sqrt{4 \pi})$, then the upper limit of $M_{R}$ can be higher.

In the numerical calculation, we generate randomly the parameters in the chosen ranges and do the numerical integrations in the formulae of $\widetilde{S}, \widetilde{T}$ of the 
scalars and the mirror fermions as expressed in Eqs. (4.3.2, 4.3.3, 4.3.4, 4.3.5, $4.4 .1,4.4 .2)$.

\subsubsection{The Unconstrained $S, T$}

In this section, we present the $\widetilde{S}, \widetilde{T}$ of the scalar and the fermionic sectors in the whole parameter space.

- The scatter plot below presents the contribution of New Physics to the oblique parameters of the mirror fermions.

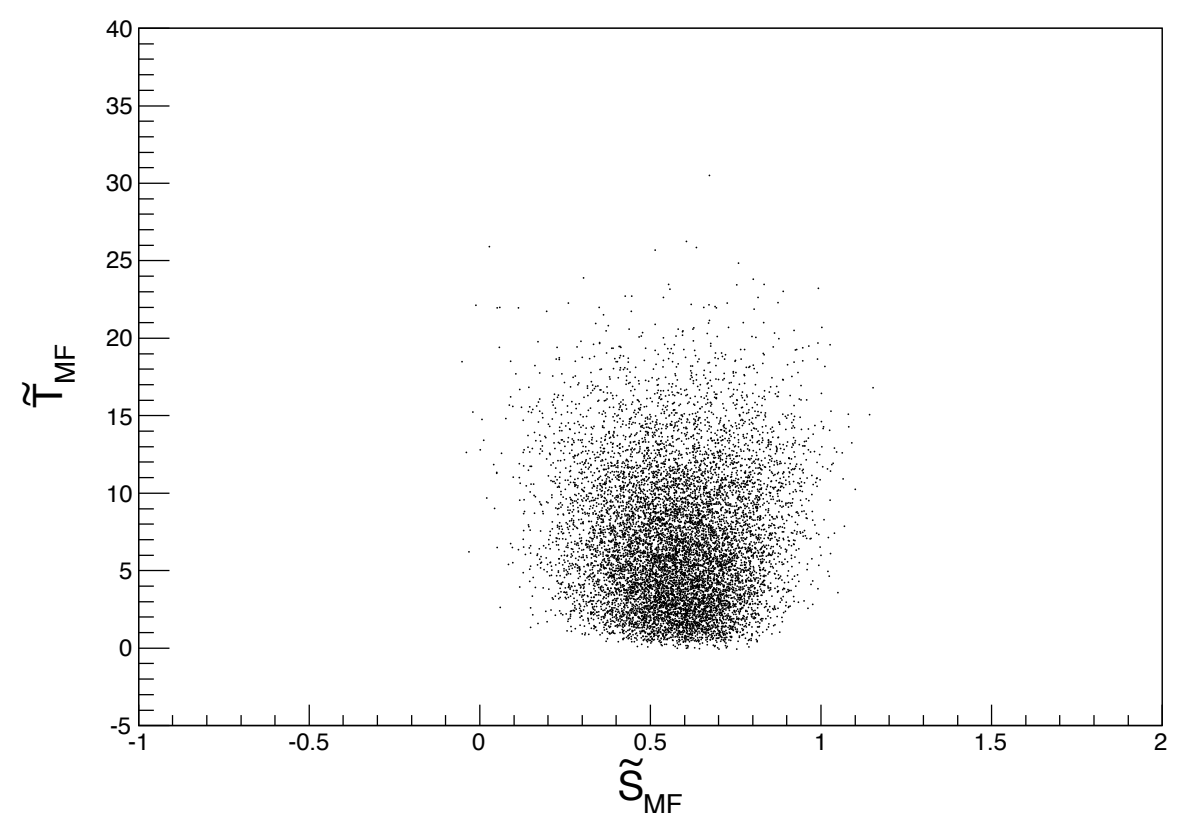

Figure 4.3. The contribution of the mirror fermions.

In general, the new chiral doublets produce positive contributions to the oblique parameters. As shown in Fig. 4.3, $\widetilde{T}_{M F}$ can be very positively large depending on the mass splitting between the member of each family. To compare with the electroweak precision measurements, we include the experimental 1, $2 \sigma$ contours 


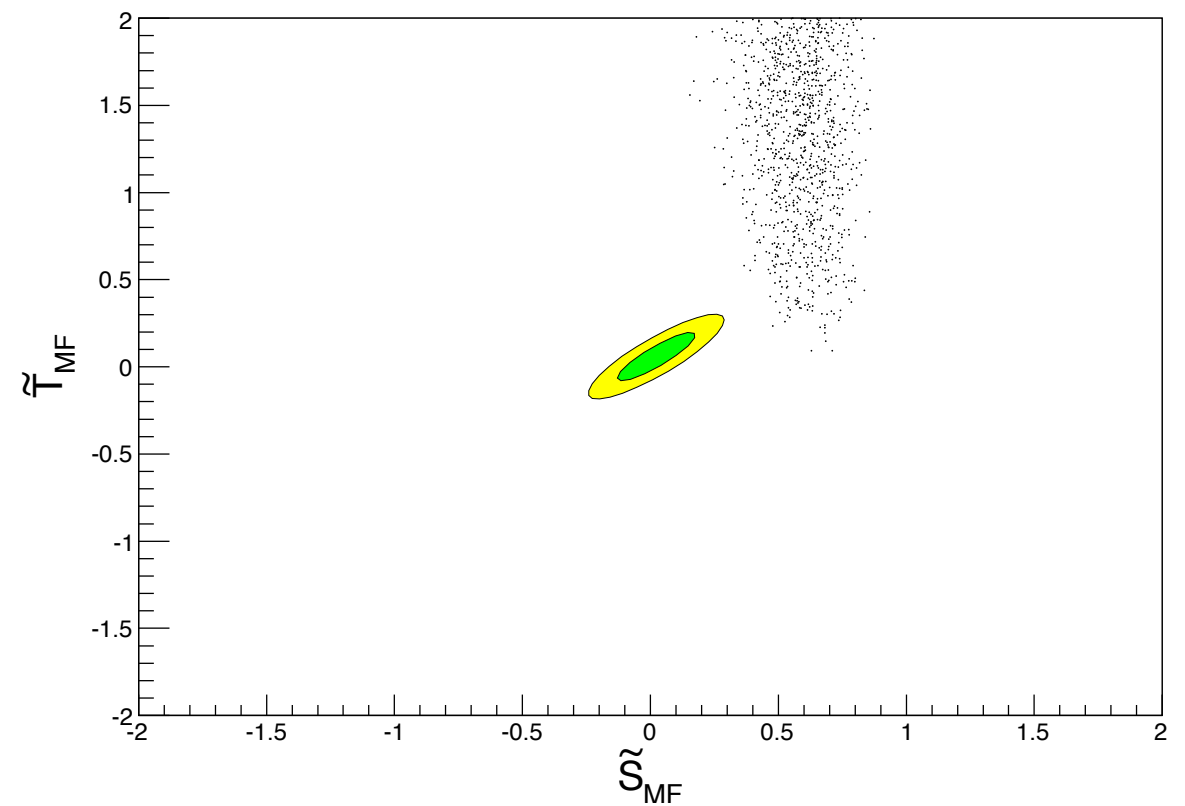

Figure 4.4. The contribution of the mirror fermions is always above the $2-\sigma$ constraint. Here the EW precision measurements constraints are $\widetilde{S}=$ $-0.02 \pm 0.14, \widetilde{T}=0.06 \pm 0.14[23]$

Eventually, if there were just mirror fermions in the model, it was impossible to find a solution in which $\widetilde{S}, \widetilde{T}$ satisfy the electroweak precision constraints as seen in the Fig. 4.4.

- With all the masses of the scalar varied from $M_{Z} / 2$ to $650 \mathrm{GeV}$, we have the contributions of the scalar sector to the oblique parameters in Fig. 4.5. 


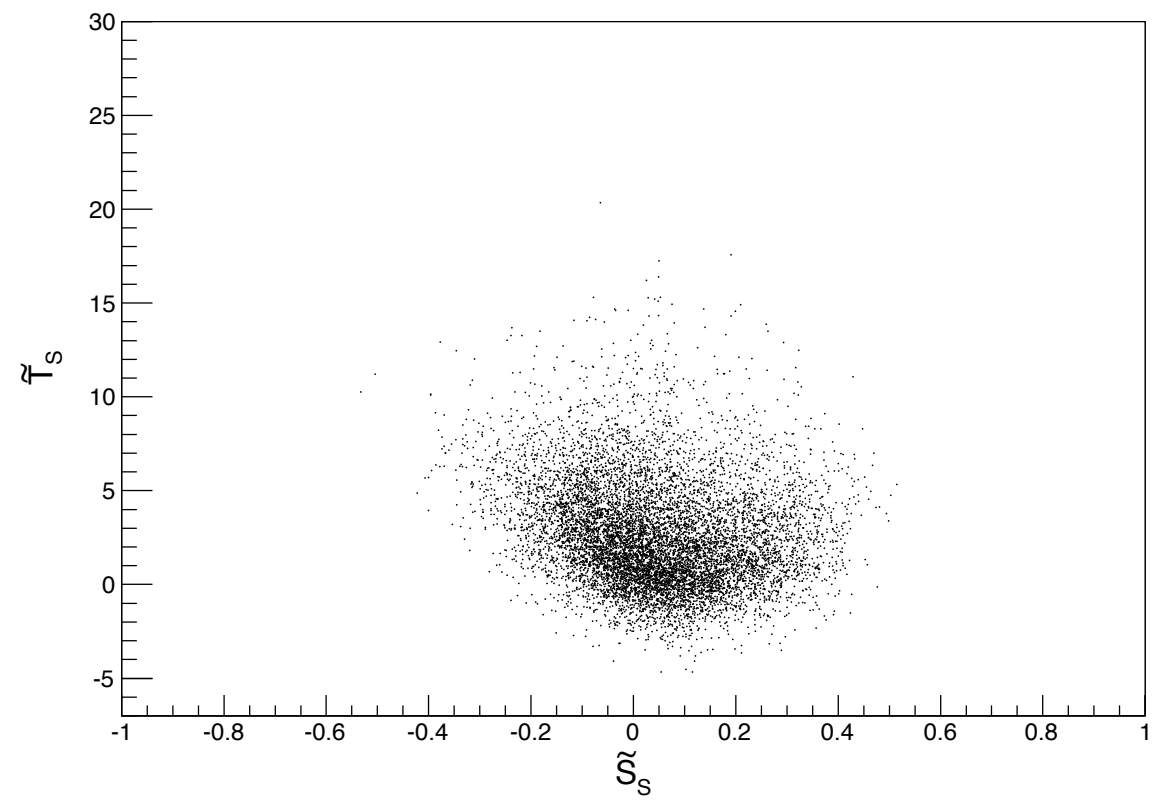

Figure 4.5. The contribution of the scalars.

As we see, there is a certain region of parameter space of the model where $\tilde{S}_{S}$ and $\tilde{T}_{S}$ can be very negative. It can be clearly seen when the EW precision contours are added in the Fig. 4.6. Here,we just plot in the region of $-2 \leq \tilde{S}_{S}, \tilde{T}_{S} \leq 2$ for the purpose of illustration. 


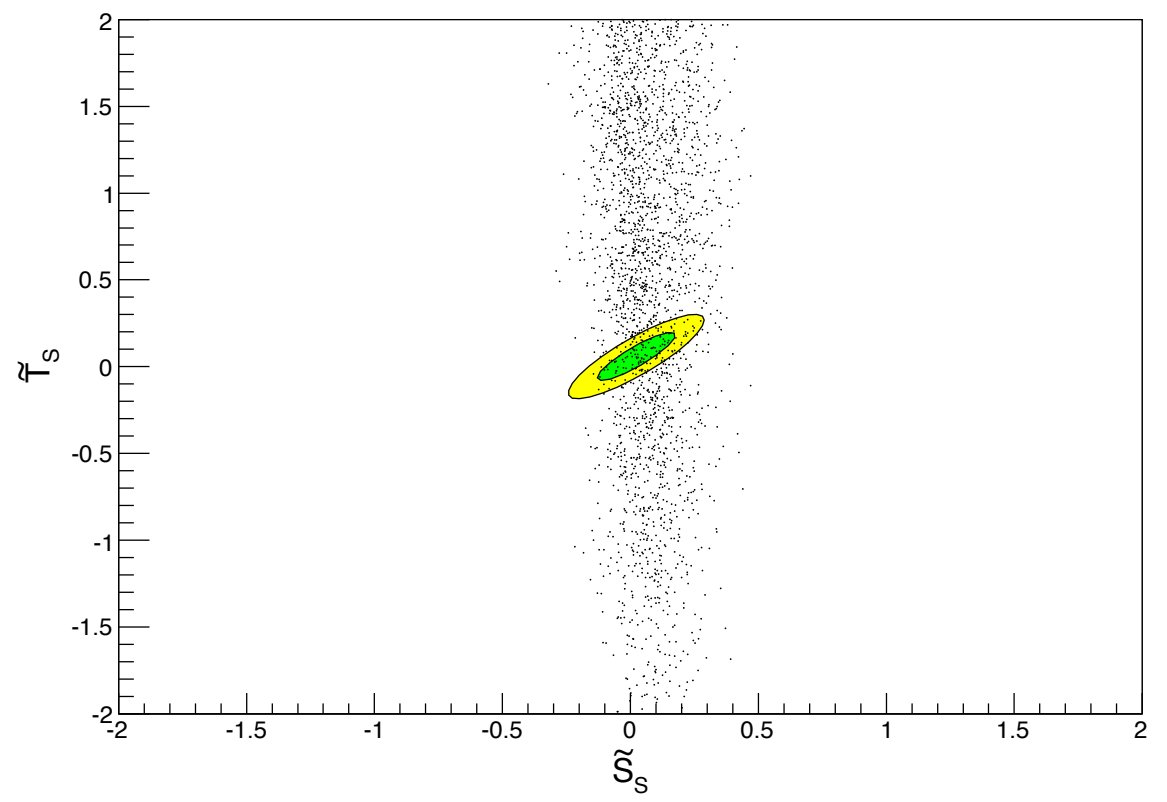

Figure 4.6. The contribution of the scalars with the EW precision measurement constraints.

In conclusion, the mirror fermions in the $\mathrm{EW} \nu_{R}$ model make positive contributions to both $S$ and $T$ as expected for any extra chiral doublet. On the other hand, the new scalars in the model eventually contribute negatively to these parameters in a certain parameter space. We expect a cancellation between two sectors in the oblique parameters to ensure that the total $S$ and $T$ satisfy the electroweak precision measurement constraints.

\subsubsection{The Constrained $S$ and $T$}

To apply the constraints from the electroweak precision measurements, we combine the contributions of the mirror fermions and the new scalars as expressed in Eqs. $(4.1 .8,4.1 .9)$. The final formulas of $\widetilde{S}$ and $\widetilde{T}$ contain non-analytical 
integrals. A $\mathrm{C}++$ program was written to calculate these integrals numerically. We found the allowed region of the $\mathrm{EW} \nu_{R}$ model in which the total contribution of the NP satisfies the experimental constraints as shown in Fig. 4.7.

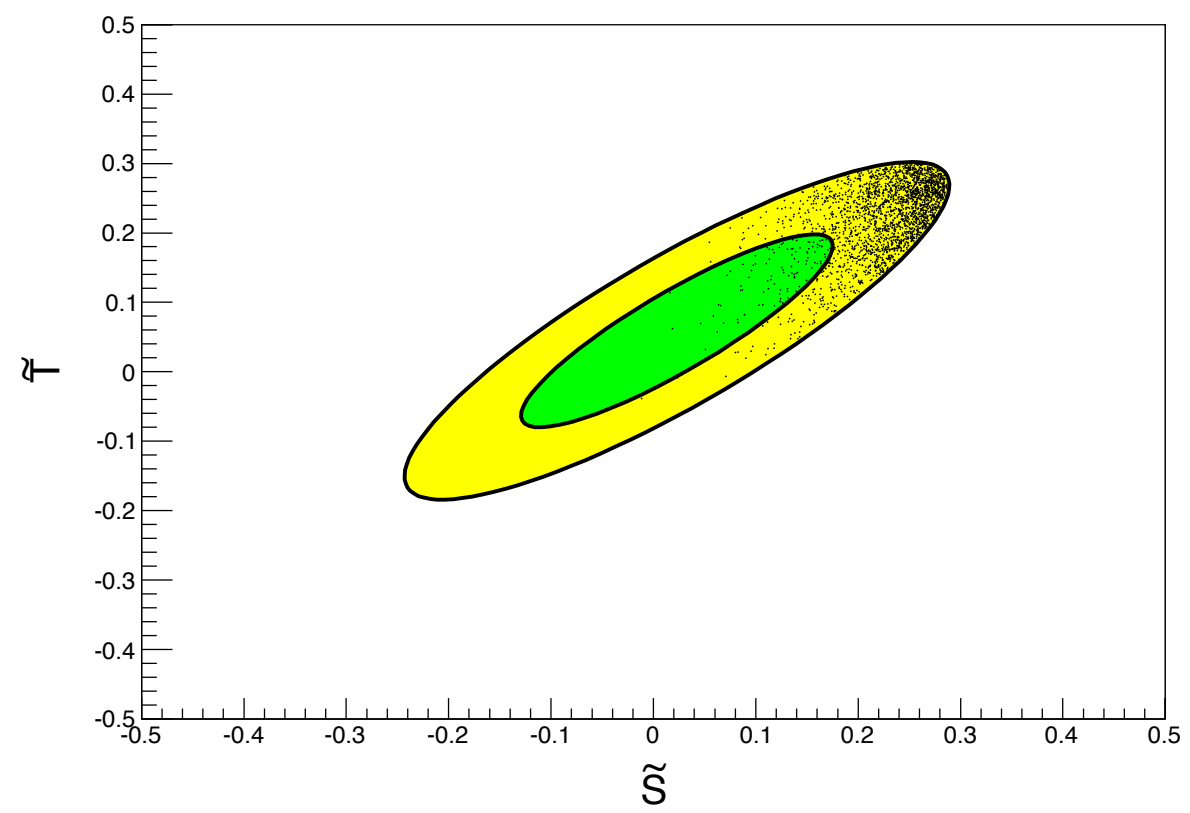

Figure 4.7. The total $\widetilde{T}$ vs $\widetilde{S}$ with the 1 and $2 \sigma$ experimental contours.

In Fig. 4.7, there are 100 points within the $1 \sigma$ region among 3000 points within the $2 \sigma$ region. Here, the number of points is a representative quantity to prove the cancellation between the contributions of the mirror fermions and the new scalars in the $\mathrm{EW} \nu_{R}$ model. The more time allocated to the computational calculation, the more points in the 1 and $2 \sigma$ regions we achieve. It indicates that there is a region of the parameter space where the $\mathrm{EW} \nu_{R}$ model is consistent with the electroweak precision measurements.

To explore the cancellation between the scalars and the mirror fermions in the 
model, we investigate the correlation of the contributions to $S, T$ separately in the allowed region.

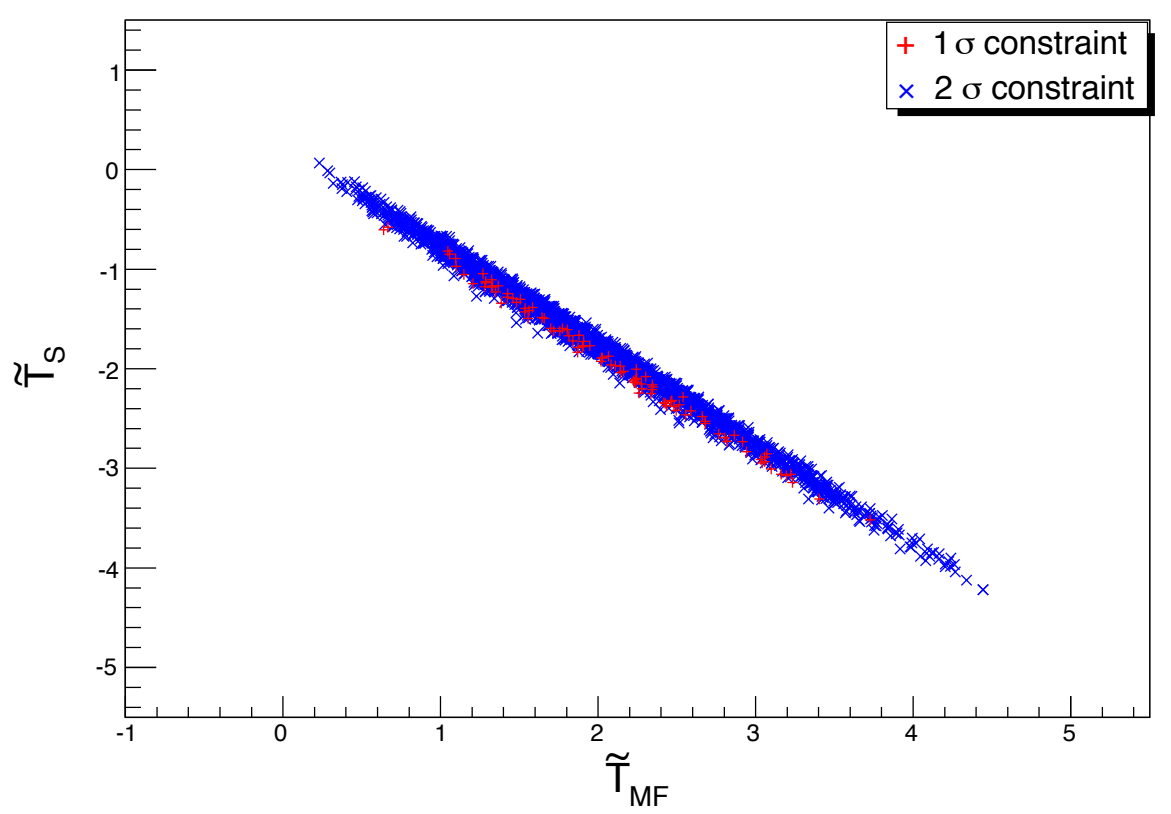

Figure 4.8. $\widetilde{T}_{S}$ vs $\widetilde{T}_{M F}$ under the 1 and $2 \sigma$ experimental constraints. 


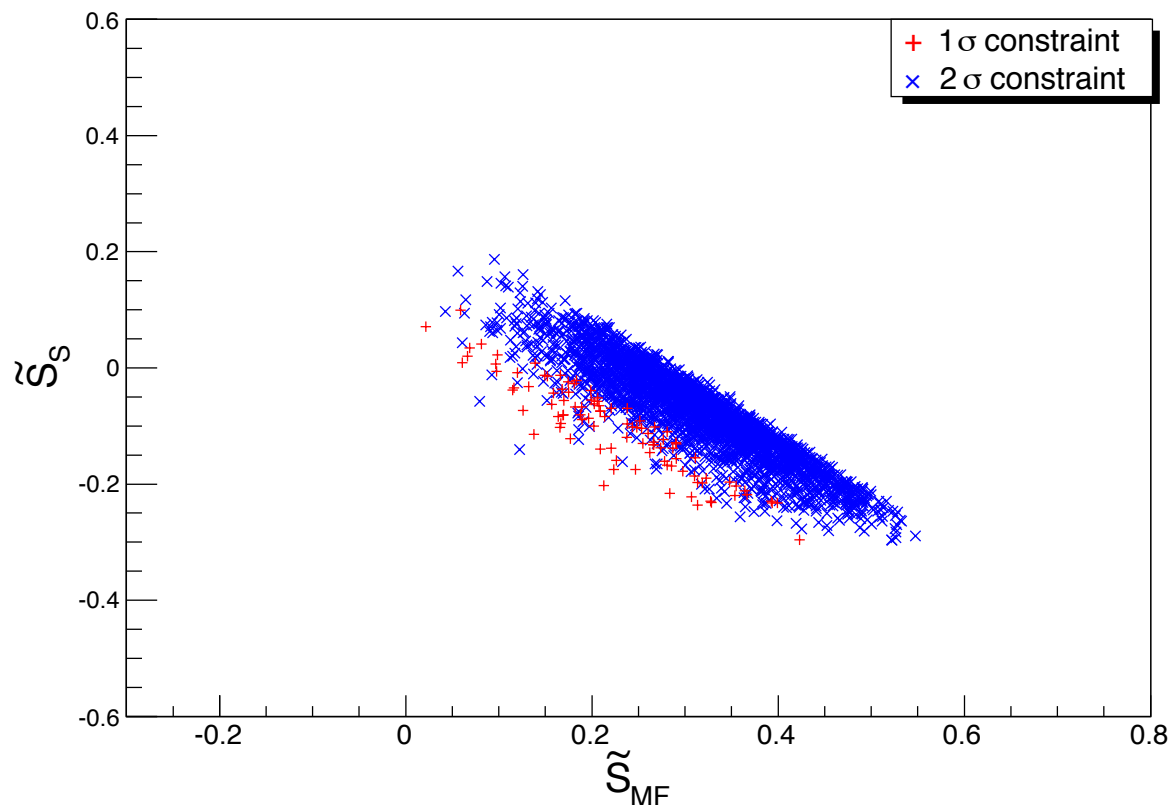

Figure 4.9. $\quad \widetilde{S}_{S}$ vs $\widetilde{S}_{M F}$ under the 1 and $2 \sigma$ experimental constraints.

From Fig. 4.8 and Fig. 4.9, it is clear to see the cancellation between the contributions to $\widetilde{S}$ and $\widetilde{T}$ of the scalar and the mirror fermions. The positive $\widetilde{T}_{M F}$ is canceled by the negative $\widetilde{T}_{S}$ so that the total $\widetilde{T}$ satisfies the 1 and $2 \sigma$ constraints. The more negative the $\widetilde{T}_{S}$ and $\widetilde{S}_{S}$ is, the more positive of $\widetilde{T}_{M F}$ and $\widetilde{S}_{M F}$ can be.

Lastly, we also investigate the dependence of $\widetilde{T}$ and $\widetilde{S}$ on the mixing angle in the scalar sector. 


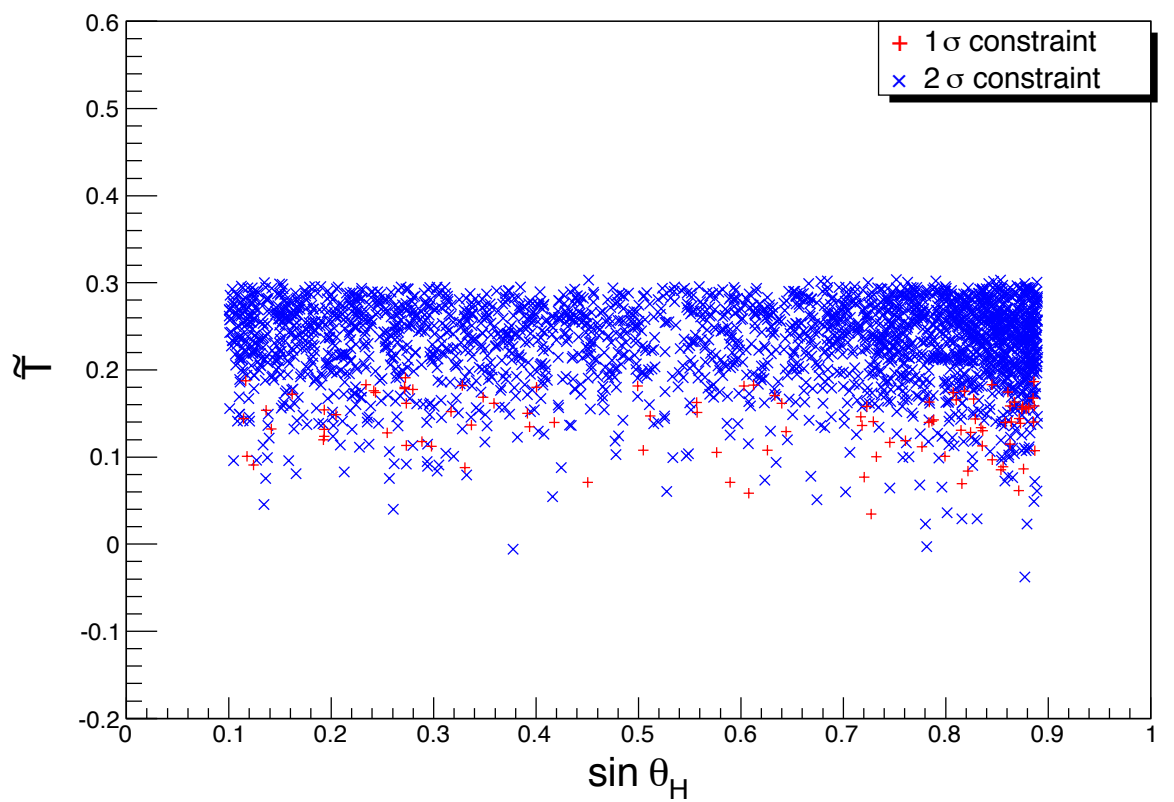

Figure 4.10. $\widetilde{T}$ vs $\sin \theta_{H}$ under the 1 and $2 \sigma$ experimental constraints. 


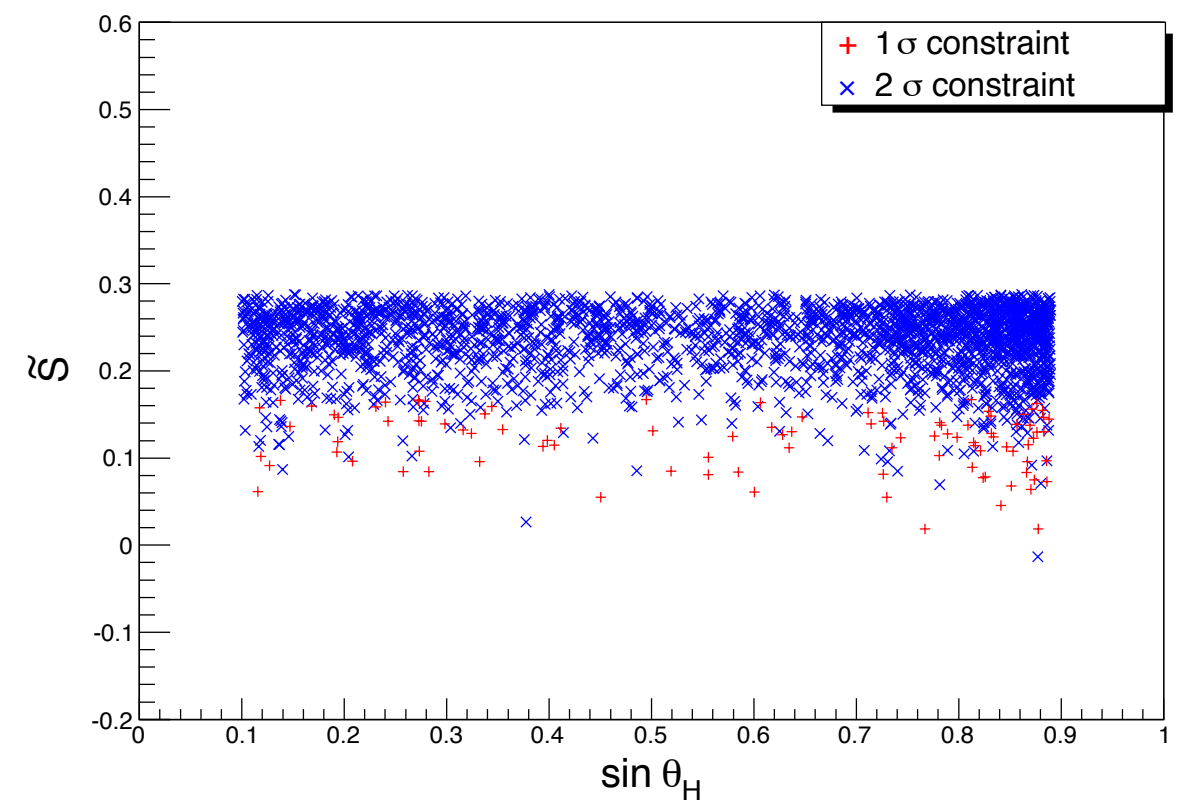

Figure 4.11. $\widetilde{S}$ vs $\sin \theta_{H}$ under the 1 and $2 \sigma$ experimental constraints.

From Fig. 4.10 and Fig. 4.11, the model is consistent with the electroweak precision measurements in the whole range of the mixing angle. It implies that the contributions of New Physics of the model to $\widetilde{T}, \widetilde{S}$ can satisfy the electroweak precision measurements at any ratio of the VEVs of the scalar doublet $\Phi$ and the scalar triplet $\chi$.

\subsubsection{The Dependence of $\widetilde{T}_{S}$ and $\widetilde{S}_{S}$ On the Mass Splittings}

To understand the canceling mechanism between the contributions to the oblique parameter of the scalars and the mirror fermions, it is important to investigate the dependence of the $\widetilde{S}_{S}, \widetilde{T}_{S}$ on the mass splitting between the singlycharged and the neutral scalars in the triplet $H_{3}$ and the quintet $H_{5}$. In this case, the masses of the singly-charged scalars, $H_{3}^{+}, H_{5}^{+}$, are varied from $M_{Z}$ to 
$650 \mathrm{GeV}$. The other parameters, $\sin \theta_{H}, m_{H_{5}^{0,++}}, m_{H_{3}^{0}}, m_{H_{1}^{0}}, m_{H_{1}^{0 \prime}}$ are fixed.

- For the small mixing angle $\sin \theta_{H}=0.1$.
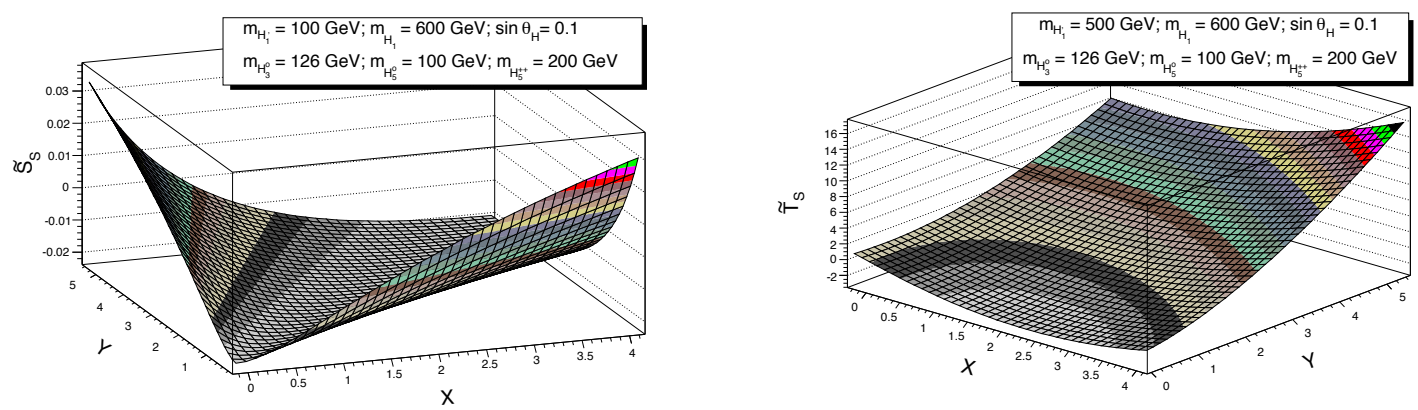

Figure 4.12. The dependence of $\widetilde{T}_{S}, \widetilde{S}_{S}$ on $X=1-\frac{m_{H_{3}^{+}}}{m_{H_{3}^{0}}}$ and $Y=1-\frac{m_{H_{5}^{+}}}{m_{H_{5}^{0}}}$ at $m_{H_{5}^{++}}=200 \mathrm{GeV}$
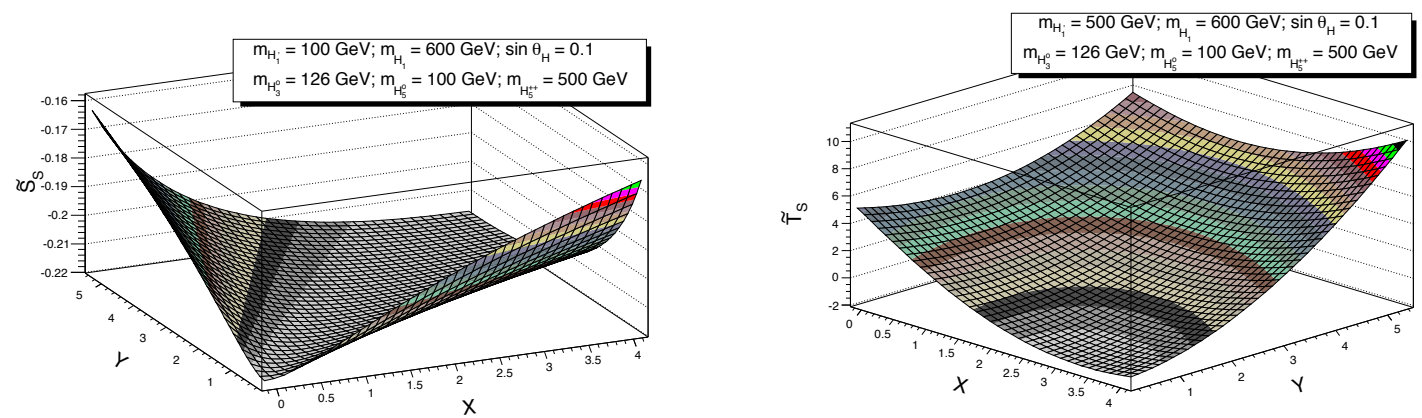

Figure 4.13. The dependence of $\widetilde{T}_{S}, \widetilde{S}_{S}$ on $X=1-\frac{m_{H_{3}^{+}}}{m_{H_{3}^{0}}}$ and $Y=1-\frac{m_{H_{5}^{+}}}{m_{H_{5}^{0}}}$ at $m_{H_{5}^{++}}=500 \mathrm{GeV}$

- For the large mixing angle $\sin \theta_{H}=0.89$. 

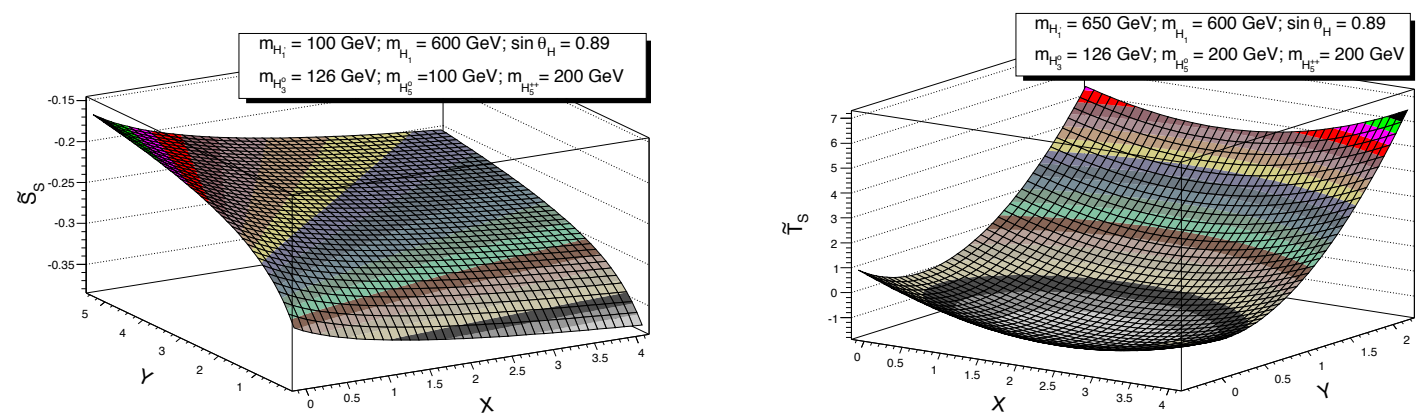

Figure 4.14. The dependence of $\widetilde{T}_{S}, \widetilde{S}_{S}$ on $X=1-\frac{m_{H_{3}^{+}}}{m_{H_{3}^{0}}}$ and $Y=1-\frac{m_{H_{5}^{+}}}{m_{H_{5}^{0}}}$ at $m_{H_{5}^{++}}=200 \mathrm{GeV}$
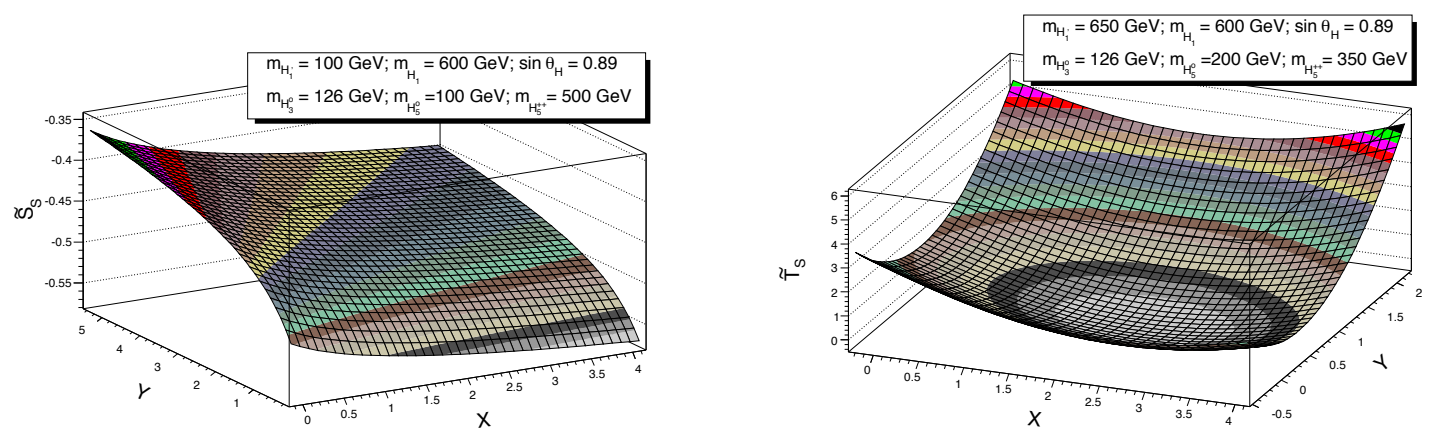

Figure 4.15. The dependence of $\widetilde{T}_{S}, \widetilde{S}_{S}$ on $X=1-\frac{m_{H_{3}^{+}}}{m_{H_{3}^{0}}}$ and $Y=1-\frac{m_{H_{5}^{+}}}{m_{H_{5}^{0}}}$ at $m_{H_{5}^{++}}=500 \mathrm{GeV}$ for $\widetilde{S}_{S}$ and $m_{H_{5}^{++}}=350 \mathrm{GeV}$ for $\widetilde{T}_{S}^{3}$

\section{* Remarks}

- In this analysis, the masses of the two singlet $H_{1}^{0}, H_{1}^{0 \prime}$ are chosen arbitrarily at $100,600,650 \mathrm{GeV}$ without losing the generality. We still see the influence 
of these singlets on $\widetilde{T}_{S}$ and $\widetilde{S}_{S}$ at the different ranges of their masses. The pseudo-scalar $H_{3}^{0}$ is chosen at mass $m_{H_{3}^{0}}=126 \mathrm{GeV}$ for illustration purpose. In the next chapter, we present a complete analysis about incorporating the discovered SM-like Higgs boson into the $\mathrm{EW} \nu_{R}$ model.

- In both cases of the mixing angle $\sin \theta_{H}=0.1,0.89$, as seen in Figs. 4.12, $4.13,4.14,4.15, \widetilde{S}_{S}$ tends to be more negative when the mass of doublycharged scalar $\mathrm{H}_{5}^{++}$increases. At the same time, the mass splitting of $H_{5}^{+}, H_{5}^{0}$ must be larger to ensure that $\widetilde{T}_{S}$ is negative enough to cancel the positive contribution of the mirror fermions.

- At the same mass of $H_{5}^{++}, \widetilde{T}_{S}$ and $\widetilde{S}_{S}$ tend to be more negative when the mixing angle $\theta_{H}$ increasing as seen in Figs. 4.12, 4.14.

- For the small mixing angle case, $\sin \theta_{H}=0.1$ in Figs. 4.12, 4.13, $\widetilde{S}_{S}$ decreases as the mass splittings between $H_{3}^{+}, H_{3}^{0}$ and between $H_{5}^{+}, H_{5}^{0}$ become similar.

- For the large mixing angle case, $\sin \theta_{H}=0.89$ in Figs. 4.14, 4.15, $\widetilde{S}_{S}$ decreases as $H_{5}^{+}, H_{5}^{0}$ become more degenerate, while $H_{3}^{+}$becomes heavier than $H_{3}^{0}$. This is the different behavior in comparing with the small mixing angle case.

These observations are very useful in the search for the signals of the new scalars in the $\mathrm{EW} \nu_{R}$ model. The constraints of the electroweak precision measurements restrict the mass splitting in the scalar sector which indicates the decay products of the scalars of the model at high energy colliders.

\subsection{Summary of The Chapter}

The study of physics at electroweak scale is well established. Any new physics is constrained tightly through so-called oblique parameters, $S$ and $T$ defined in Eq. 
(4.1.6). These parameters basically tell us how much contribution of new physics to the experimental data is allowed. This is one of the most important tests for any Beyond the Standard Model phenomena. Indeed, many models fail to satisfy the electroweak precision measurements data or are constrained to a very small parameter space.

On the contrary, we have shown that the model of right-handed neutrinos at electroweak scale $\left(E W \nu_{R}\right)$ well satisfies the precision measurements constraints with a sizable parameter space. In general, including any new chiral doublet has a consequence of contributing positively in the oblique parameters, especially the $S$ parameter. As shown in Figs. 4.3, 4.4, the additional mirror quarks and leptons can have a hugely positive contribution to the oblique parameters. Eventually, there would be no allowed region of the parameter space if the $\mathrm{EW} \nu_{R}$ model just contained the mirror fermions. However, the model also has the new scalars which can offset the positive contribution of the mirror fermions. The original purpose of introducing the scalar triplets is to generate the mass of right-handed neutrinos at electroweak scale and to preserve the custodial symmetry at the tree level. As pointed out in [24], these scalar triplets generally contribute negatively to the $S$ parameter so that the total contribution of new physics of the $E W \nu_{R}$ model to the oblique parameters satisfies the electroweak precision measurements. We here have provided a detailed calculation to prove that statement.

The contribution of new physics in the scalar sector of the $\mathrm{EW} \nu_{R}$ model has been presented in Figs. 4.5, 4.6. It is clear to see that the $\widetilde{S}, \widetilde{T}$ of the scalar sector has a large part in the negative region. As expected, the sum of the contributions of the mirror fermions and the new scalars satisfies the experimental constraints of the electroweak precision measurements at the 1 and $2 \sigma$ contours in Fig. 4.7. In more details, the correlations between the two contributions to the total $\widetilde{S}$ and $\widetilde{T}$ have been shown in Figs. 4.9, 4.8 to illustrate how the cancellation works in 
the EW $\nu_{R}$ model. In short, the negative regions of the $\widetilde{S}_{S}$ and $\widetilde{T}_{S}$ cancel out the positive regions of $\widetilde{S}_{M F}$ and $\widetilde{T}_{M F}$.

We have shown the wide range of the parameter space where the $\mathrm{EW} \nu_{R}$ model satisfies the electroweak precision constraints. As an illustrative example, we have presented the dependence of $\widetilde{S}$ and $\widetilde{T}$ on the mixing angle in the scalar sector $\theta_{H}$. In Figs. $4.10,4.11$, it is clear that the model satisfies the constraints in the whole range of $\sin \theta_{H}$. We have also shown how the mass splittings, in particular in the scalar sector, affect the values of $\widetilde{S}$ and $\widetilde{T}$, in Figs. 4.12, 4.13, 4.14, 4.15. The contribution to $\widetilde{S}, \widetilde{T}$ of the scalar sector correlates with the contribution of the fermionic sector as shown in Figs.4.9, 4.8. As the result, the mass splittings among the scalars decide how much the mass splittings between mirror fermions could be. The study about theses mass splittings would be very important for the search for the signals of the $\mathrm{EW} \nu_{R}$ model at hadron colliders.

As for now, we can conclude that the model of right-handed neutrinos at electroweak scale passes the first test of the experimental results. The next step would be to study the model in light of the $125 \mathrm{GeV}$ Standard Model-like Higgs boson discovery. 


\section{Chapter 5}

\section{The Extended EW $\nu_{R}$ Model}

\subsection{The $\mathrm{EW} \nu_{R}$ Model in the Light of $125 \mathrm{GeV}$ Higgs Discovery}

\subsubsection{The $125 \mathrm{GeV}$ Higgs Boson}

After the Higgs discovery [26], one needs to find the candidate for the $125 \mathrm{GeV}$ SM-like Higgs boson of the $\mathrm{EW} \nu_{R}$ model. What are the characteristics of the $125 \mathrm{GeV}$ SM-like Higgs boson?

- It is a resonance raising above the background in the major decay channels at the LHC such as, $W W^{*}, Z Z^{*}, \gamma \gamma, \tau^{+} \tau-, b \bar{b}$.

- The new particle decays into two photons, so it disfavors spin one nature based on the Landau-Yang theorem.

- The decays to pairs vector bosons indicate that the new particle is electrically neutral.

- Its existence is determined through the signal strength calculated by

$$
\mu=\frac{\sigma \cdot B r(H \rightarrow X X)_{d a t a}}{\sigma \cdot B r\left(H_{S M} \rightarrow X X\right)}
$$


In the newest result, the signal strength of the $125 \mathrm{GeV}$ SM-like Higgs boson is $[27]$

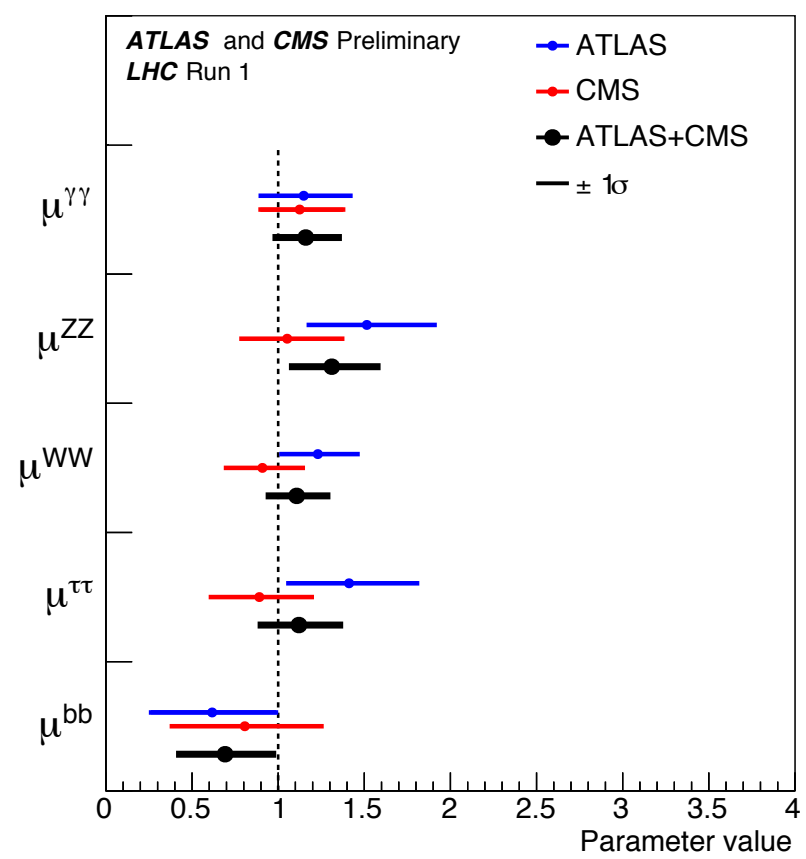

Figure 5.1. The measured signal strength of the $125 \mathrm{GeV}$ SM-like Higgs boson at the LHC

- Spin-Parity analysis shows that the new boson is favor to be a CP-even spin-zero state by more than $3 \sigma[28]$.

\subsubsection{The $125 \mathrm{GeV}$ Higgs Boson Candidate of The Original $\mathrm{EW} \nu_{R}$ Model}

In the original $\mathrm{EW} \nu_{R}$ model, the mass spectrum of the scalar sector consists four neutral spin-zero states, $H_{1}^{0}, H_{1}^{0 \prime}, H_{3}^{0}, H_{5}^{0}$. Among those, $H_{3}^{0}, H_{5}^{0}$ have the CP-odd nature. Thus that they cannot be the candidate for the $125 \mathrm{GeV}$ Higgs boson due to the exclusion from the spin-parity analysis of ATLAS and CMS [28]. 
While $H_{1}^{0}, H_{1}^{0 \prime}$ are the CP-even states of the model. $H_{1}^{0 \prime}$ is purely made from the scalar triplets $\vec{\chi}, \xi$. Then it does not couple to the SM fermions. As the result, $H_{1}^{0 \prime}$ cannot be the candidate neither. It leaves $H_{1}^{0}$ to be the only potential choice.

At the LHC, the Higgs boson is produced mainly through gluon fusion which is the triangle loop with the heavy quarks inside. In the minimal $\mathrm{EW} \nu_{R}$ model, there are six mirror quarks with the masses in the same order of magnitude with the mass of top quark. The CP-even state $H_{1}^{0}$ couples to both the mirror quarks and the SM quarks in a form of $g_{H_{1}^{0} q \bar{q}}=-\imath \frac{m_{q} g}{2 m_{W} c_{H}}$. Therefore, there is an approximate factor of $\frac{49}{c_{H}^{2}}$ coming from the ratio of the production cross-section of $H_{1}^{0}$ and that of $H_{S M}$ in the signal strength of the $H_{1}^{0}$. The factor of 49 is because of the contribution of 6 mirror quarks and 1 top quark into the gluon fusion of $H_{1}^{0}$ $\left((6+1)^{2}\right)$. While $c_{H}<1$ as always. As the result, there is a huge enhancement in the production cross-section which cannot be compensated by the ratio of the branching ratio in any case. It means that the signal strength of $H_{1}^{0}$ is always higher than the ATLAS/CMS measurements which are very close to 1.

\subsection{The Extension}

As stated in the last section, the most plausible candidate for the $125 \mathrm{GeV}$ Higgs boson is ruled out due to the large enhancement in the production crosssection via gluon fusion. The reason is that the $H_{1}^{0}$ couples to the mirror quarks and the SM quarks in the same way, $g_{H_{1}^{0} q \bar{q}}=-\imath \frac{m_{q} g}{2 m_{W} c_{H}}$. To avoid that scenario, we want to expand the original $\mathrm{EW} \nu_{R}$ model so that the mirror fermions and the SM fermions have a different way to couple to the scalars.

The natural way is to expand the scalar sector by adding one more complex scalar doublet, called $\Phi_{2 M}$, because the new scalar doublet preserves the custodial symmetry at tree level [29]. We then have two complex scalar doublets, $\Phi_{2}, \Phi_{2 M}$, in the extended version of the EW $\nu_{R}$ model, which will be called as the EW $\nu_{R}$ 
model from now on. Then we name the original $\mathrm{EW} \nu_{R}$ as the minimal model in the sense that the scalar spectrum in the original version is the minimal choice for generating the mass of neutrinos. Among these two doublets, $\Phi_{2}$ only couples to the Standard Model fermions, while $\Phi_{2 M}$ only couples to the mirror fermions. To prevent the cross coupling, we also impose a global symmetry $U(1)_{S M} \times U(1)_{M F}$ such that

$$
\begin{gathered}
U(1)_{S M}: \quad \Phi_{2} \rightarrow e^{\imath \alpha_{S M}} \Phi_{2} \\
\left(q_{L}^{S M}, l_{L}^{S M}\right) \rightarrow e^{\imath \alpha_{S M}}\left(q_{L}^{S M}, l_{L}^{S M}\right), \\
U(1)_{M F} \quad: \quad \Phi_{2 M} \rightarrow e^{\imath \alpha_{M F}} \Phi_{2 M} \\
\quad\left(q_{R}^{M}, l_{R}^{M}\right) \rightarrow e^{\imath \alpha_{M F}}\left(q_{R}^{M}, l_{R}^{M}\right), \\
\phi_{S} \rightarrow e^{-\imath\left(\alpha_{M F}-\alpha_{S M}\right)} \phi_{S}, \\
\tilde{\chi} \rightarrow e^{-2 \imath \alpha_{M F}} \tilde{\chi} .
\end{gathered}
$$

All other fields such as $S U(2)$ singlet right-handed SM fermions, left-handed mirror fermions are singlets under the $U(1)_{S M} \times U(1)_{M F}$ group.

Under this global symmetry, the Yukawa couplings such as $g_{Y} \bar{f}_{L} \Phi_{2} M f_{R}$ and $g_{Y} \bar{f}_{R}^{M} \Phi_{2} f_{L}^{M}$ are forbidden at tree level. Only the Yukawa interactions of the type $g_{Y} \bar{f}_{L} \Phi_{2} f_{R}$ and $g_{Y} \bar{f}_{R}^{M} \Phi_{2 M} f_{L}^{M}$ are allowed. The Yukawa couplings of the physical scalars to SM and mirror fermions will involve in the mixing angles.

\subsection{The Spontaneous Symmetry Breaking}

Having added one more complex scalar doublet, the scalar sector of the EW $\nu_{R}$ model now consists two $\mathrm{Y}=1$ scalar doublets and a $\mathrm{Y}=0$ scalar triplet $(\xi)$ and 
a $\mathrm{Y}=3$ scalar triplet $(\vec{\chi})$ besides the scalar singlet $\Phi_{S}$. In the presentations of the global $S U(2)_{L} \times S U(2)_{R}$, these doublets and triplets have the form of

$$
\begin{aligned}
\Phi_{2} & =\left(\begin{array}{cc}
\phi_{2}^{0, *} & \phi_{2}^{+} \\
\phi_{2}^{-} & \phi_{2}^{0}
\end{array}\right), \\
\Phi_{2 M} & =\left(\begin{array}{cc}
\phi_{2 M}^{0, *} & \phi_{2 M}^{+} \\
\phi_{2 M}^{-} & \phi_{2 M}^{0}
\end{array}\right),
\end{aligned}
$$

and

$$
\chi=\left(\begin{array}{ccc}
\chi^{0} & \xi^{+} & \chi^{++} \\
\chi^{-} & \xi^{0} & \chi^{+} \\
\chi^{--} & \xi^{-} & \chi^{0 *}
\end{array}\right)
$$

Then the kinetic part of the Lagrangian of the Higgs sector is:

$$
\begin{aligned}
\mathcal{L}_{K H} & =\frac{1}{2} \operatorname{Tr}\left[\left(D_{\mu} \Phi_{2}\right)^{\dagger}\left(D^{\mu} \Phi_{2}\right)\right]+\frac{1}{2} \operatorname{Tr}\left[\left(D_{\mu} \Phi_{2 M}\right)^{\dagger}\left(D^{\mu} \Phi_{2 M}\right)\right]+ \\
& +\frac{1}{2} \operatorname{Tr}\left[\left(D_{\mu} \chi\right)^{\dagger}\left(D^{\mu} \chi\right)\right]+\left|\partial_{\mu} \phi_{S}\right|^{2} .
\end{aligned}
$$

Here, the derivatives of the scalars are expressed as

$$
\begin{aligned}
D_{\mu} \Phi & =\partial_{\mu} \Phi_{2}+i g \vec{W} \frac{\vec{\tau}}{2} \Phi_{2}-i g^{\prime} \Phi B \frac{\tau_{3}}{2} \\
D_{\mu} \Phi_{2 M} & =\partial_{\mu} \Phi_{2 M}+i g \vec{W} \frac{\vec{\tau}}{2} \Phi_{2 M}-i g^{\prime} \Phi_{2 M} B \frac{\tau_{3}}{2} \\
D_{\mu} \chi & =\partial_{\mu} \chi+i g \vec{W} \frac{\vec{t}}{2} \chi-i g^{\prime} \chi B \frac{t_{3}}{2} .
\end{aligned}
$$


In the most general case, the $S U(2)_{L} \times S U(2)_{R}$ invariant potential of $\Phi_{2}, \Phi_{2 M}, \chi$ has the form of

$$
\begin{aligned}
V\left(\Phi_{2}, \Phi_{2 M}, \chi\right)= & \lambda_{1}\left[\operatorname{Tr} \Phi_{2}^{\dagger} \Phi_{2}-v_{2}^{2}\right]^{2}+\lambda_{2}\left[\operatorname{Tr} \Phi_{2 M}^{\dagger} \Phi_{2 M}-v_{2 M}^{2}\right]^{2}+\lambda_{3}\left[\operatorname{Tr} \chi^{\dagger} \chi-3 v_{M}^{2}\right]^{2} \\
& +\lambda_{4}\left[\operatorname{Tr} \Phi_{2}^{\dagger} \Phi_{2}-v_{2}^{2}+\operatorname{Tr} \Phi_{2 M}^{\dagger} \Phi_{2 M}-v_{2 M}^{2}+\operatorname{Tr} \chi^{\dagger} \chi-3 v_{M}^{2}\right]^{2} \\
& +\lambda_{5}\left[\left(\operatorname{Tr} \Phi_{2}^{\dagger} \Phi_{2}\right)\left(\operatorname{Tr} \chi^{\dagger} \chi\right)-2\left(\operatorname{Tr} \Phi_{2}^{\dagger} \frac{\tau^{a}}{2} \Phi_{2} \frac{\tau^{b}}{2}\right)\left(\operatorname{Tr} \chi^{\dagger} T^{a} \chi T^{b}\right)\right] \\
& +\lambda_{6}\left[\left(\operatorname{Tr} \Phi_{2 M}^{\dagger} \Phi_{2 M}\right)\left(\operatorname{Tr} \chi^{\dagger} \chi\right)-2\left(\operatorname{Tr} \Phi_{2 M}^{\dagger} \frac{\tau^{a}}{2} \Phi_{2 M} \frac{\tau^{b}}{2}\right)\left(\operatorname{Tr} \chi^{\dagger} T^{a} \chi T^{b}\right)\right] \\
& +\lambda_{7}\left[\left(\operatorname{Tr} \Phi_{2}^{\dagger} \Phi_{2}\right)\left(\operatorname{Tr} \Phi_{2 M}^{\dagger} \Phi_{2 M}\right)-\left(\operatorname{Tr} \Phi_{2}^{\dagger} \Phi_{2 M}\right)\left(\operatorname{Tr} \Phi_{2 M}^{\dagger} \Phi_{2}\right)\right] \\
& +\lambda_{8}\left[3 \operatorname{Tr} \chi^{\dagger} \chi \chi^{\dagger} \chi-\left(\operatorname{Tr} \chi^{\dagger} \chi\right)^{2}\right]
\end{aligned}
$$

The potential in Eq. (5.3.6) is invariant under the transformation $\chi \rightarrow-\chi$ as in the minimal $\mathrm{EW} \nu_{R}$ model. Moreover, it is also invariant under the global symmetry $U(1)_{S M} \times U(1)_{M F}$. To ensure that the spontaneous symmetry breaking happens, one needs to impose a condition $\lambda_{5}=\lambda_{6}=\lambda_{7}$. The proper vacuum alignments are chosen as

$$
\begin{aligned}
\left\langle\Phi_{2}\right\rangle & =\left(\begin{array}{cc}
v_{2} / \sqrt{2} & 0 \\
0 & v_{2} / \sqrt{2}
\end{array}\right), \\
\left\langle\Phi_{2 M}\right\rangle & =\left(\begin{array}{cc}
v_{2 M} / \sqrt{2} & 0 \\
0 & v_{2 M} / \sqrt{2}
\end{array}\right),
\end{aligned}
$$

and

$$
\langle\chi\rangle=\left(\begin{array}{ccc}
v_{M} & 0 & 0 \\
0 & v_{M} & 0 \\
0 & 0 & v_{M}
\end{array}\right),
$$

Here, $v_{2}, v_{2 M}, v_{M}$ are the VEVs of the real part of the neutral component of $\Phi_{2}, \Phi_{2 M}, \chi$, respectively. They satisfy the identity:

$$
v_{2}^{2}+v_{2 M}^{2}+8 v_{M}^{2}=v^{2}
$$


where $v \approx 246 \mathrm{GeV}$. We define

$$
s_{2}=\frac{v_{2}}{v} ; \quad s_{2 M}=\frac{v_{2 M}}{v} ; \quad s_{M}=\frac{2 \sqrt{2} v_{M}}{v} .
$$

Once these field acquire their VEVs, $S U(2)_{L} \times U(1)_{Y} \rightarrow U(1)_{e m}$ spontaneously. The gauge bosons get their masses $M_{W}=g v / 2$ and $M_{Z}=M_{W} / \cos \theta_{W}$, now $v=\sqrt{v_{2}^{2}+v_{2 M}^{2}+8 v_{M}^{2}} \approx 246 \mathrm{GeV}$. We still have $\rho=M_{Z} \cos \theta_{W} / M_{W}=1$. At the same time, the global $S U(2)_{L} \times S U(2)_{R} \rightarrow S U(2)_{D}$. The potential becomes

$$
\begin{aligned}
V\left(\Phi_{2}, \Phi_{2 M}, \chi\right) & =\lambda_{1}\left[\operatorname{Tr} \Phi_{2}^{\dagger} \Phi_{2}-v_{2}^{2}\right]^{2}+\lambda_{2}\left[\operatorname{Tr} \Phi_{2 M}^{\dagger} \Phi_{2 M}-v_{2 M}^{2}\right]^{2}+\lambda_{3}\left[\operatorname{Tr} \chi^{\dagger} \chi-3 v_{M}^{2}\right]^{2} \\
& +\lambda_{4}\left[\operatorname{Tr} \Phi_{2}^{\dagger} \Phi_{2}-v_{2}^{2}+\operatorname{Tr} \Phi_{2 M}^{\dagger} \Phi_{2 M}-v_{2 M}^{2}+\operatorname{Tr} \chi^{\dagger} \chi-3 v_{M}^{2}\right]^{2} \\
& +\lambda_{5}\left[\left(\operatorname{Tr} \Phi_{2}^{\dagger} \Phi_{2}\right)\left(\operatorname{Tr} \chi^{\dagger} \chi\right)-2\left(\operatorname{Tr} \Phi_{2}^{\dagger} \frac{\tau^{a}}{2} \Phi_{2} \frac{\tau^{b}}{2}\right)\left(\operatorname{Tr} \chi^{\dagger} T^{a} \chi T^{b}\right)+\right. \\
& +\left(\operatorname{Tr} \Phi_{2 M}^{\dagger} \Phi_{2 M}\right)\left(\operatorname{Tr} \chi^{\dagger} \chi\right)- \\
& -2\left(\operatorname{Tr} \Phi_{2 M}^{\dagger} \frac{\tau^{a}}{2} \Phi_{2 M} \frac{\tau^{b}}{2}\right)\left(\operatorname{Tr} \chi^{\dagger} T^{a} \chi T^{b}\right)+\left(\operatorname{Tr} \Phi_{2}^{\dagger} \Phi_{2}\right)\left(\operatorname{Tr} \Phi_{2 M}^{\dagger} \Phi_{2 M}\right) \\
& \left.-\left(\operatorname{Tr} \Phi_{2}^{\dagger} \Phi_{2 M}\right)\left(\operatorname{Tr} \Phi_{2 M}^{\dagger} \Phi_{2}\right)\right]+\lambda_{8}\left[3 \operatorname{Tr} \chi^{\dagger} \chi \chi^{\dagger} \chi-\left(\operatorname{Tr} \chi^{\dagger} \chi\right)^{2}\right]
\end{aligned}
$$

With the presence of $\Phi_{2}, \Phi_{2 M}, \chi$, the scalar sector in the EW $\nu_{R}$ model contains 17 degrees of freedom. After spontaneous symmetry breaking, besides three Nambu-Goldstone bosons $G_{3}^{ \pm, 0}$ eaten by three vector bosons $W / Z$, the physical Higgs fields are grouped into 14 mass eigenstates. Under the custodial $S U(2)_{D}$, we can classify these 14 states into 


$$
\begin{aligned}
\text { five-plet (quintet) } & \rightarrow H_{5}^{ \pm \pm}, H_{5}^{ \pm}, H_{5}^{0} ; \\
\text { triplet } & \rightarrow H_{3}^{ \pm}, H_{3}^{0} ; \\
\text { triplet } & \rightarrow H_{3 M}^{ \pm}, H_{3 M}^{0} ; \\
\text { three singlets } & \rightarrow H_{1}^{0}, H_{1 M}^{0}, H_{1}^{0 \prime}
\end{aligned}
$$

To present these N-G bosons and physical states explicitly, we follow the steps in the minimal $\mathrm{EW} \nu_{R}$ model. Firstly, we define mixing angles and auxiliary fields.

$$
\begin{aligned}
v & =\sqrt{v_{2}^{2}+v_{2 M}^{2}+8 v_{M}^{2}} \\
s_{2} & =\frac{v_{2}}{v}, s_{2 M}=\frac{v_{2 M}}{v}, s_{M}=\frac{2 \sqrt{2} v_{M}}{v}, \\
c_{2} & =\frac{\sqrt{v_{2 M}^{2}+8 v_{M}^{2}}}{v}, c_{2 M}=\frac{\sqrt{v_{2}^{2}+8 v_{M}^{2}}}{v}, \\
c_{M} & =\frac{\sqrt{v_{2}^{2}+v_{2 M}^{2}}}{v} .
\end{aligned}
$$

Thus,

$$
\begin{aligned}
s_{2}^{2}+c_{2}^{2}=s_{2 M}^{2}+c_{2 M}^{2}=s_{M}^{2}+c_{M}^{2}=1 . \\
\phi_{2}^{0} \equiv \frac{1}{\sqrt{2}}\left(v_{2}+\phi_{2}^{0 r}+\imath \phi_{2}^{0 \imath}\right), \\
\phi_{2 M}^{0} \equiv \frac{1}{\sqrt{2}}\left(v_{2 M}+\phi_{2 M}^{0 r}+\imath \phi_{2 M}^{0 \imath}\right) \\
\chi^{0} \equiv v_{M}+\frac{1}{\sqrt{2}}\left(\chi^{0 r}+\imath \chi^{0 \imath}\right) ; \\
\psi^{ \pm} \equiv \frac{1}{\sqrt{2}}\left(\chi^{ \pm}+\xi^{ \pm}\right), \quad \zeta^{ \pm} \equiv \frac{1}{\sqrt{2}}\left(\chi^{ \pm}-\xi^{ \pm}\right)
\end{aligned}
$$


Then three Nambu-Goldstone bosons are

$$
\begin{aligned}
G_{3}^{ \pm} & =s_{2} \phi_{2}^{ \pm}+s_{2 M} \phi_{2 M}^{ \pm}+s_{M} \psi^{ \pm} \\
G_{3}^{0} & =\imath\left(-s_{2} \phi_{2}^{0 \imath}-s_{2 M} \phi_{2 M}^{0 \imath}+s_{M} \chi^{0 \imath}\right) .
\end{aligned}
$$

While the physical Higgs fields can be expressed as

$$
\begin{aligned}
H_{5}^{++} & =\chi^{++}, H_{5}^{+}=\zeta^{+}, H_{5}^{0}=\frac{1}{\sqrt{6}}\left(2 \xi^{0}-\sqrt{2} \chi^{0 r}\right) \\
H_{3}^{+} & =-\frac{s_{2} s_{M}}{c_{M}} \phi_{2}^{+}-\frac{s_{2 M} s_{M}}{c_{M}} \phi_{2 M}^{+}+c_{M} \psi^{+}, \\
H_{3}^{0} & =\imath\left(\frac{s_{2} s_{M}}{c_{M}} \phi_{2}^{0 i}+\frac{s_{2 M} s_{M}}{c_{M}} \phi_{2 M}^{0 i}+c_{M} \chi^{0 i}\right), \\
H_{3 M}^{+} & =-\frac{s_{2 M}}{c_{M}} \phi_{2}^{+}+\frac{s_{2}}{c_{M}} \phi_{2 M}^{+}, \\
H_{3 M}^{0} & =\imath\left(-\frac{s_{2 M}}{c_{M}} \phi_{2}^{0 i}+\frac{s_{2}}{c_{M}} \phi_{2 M}^{0 i}\right) \\
H_{1}^{0} & =\phi_{2}^{0 r}, H_{1 M}^{0}=\phi_{2 M}^{0 r}, \\
H_{1}^{0 \prime} & =\frac{1}{\sqrt{3}}\left(\sqrt{2} \chi^{0 r}+\xi^{0}\right)
\end{aligned}
$$

with phase conventions $H_{5}^{--}=\left(H_{5}^{++}\right)^{*}, H_{5}^{-}=-\left(H_{5}^{+}\right)^{*}, H_{3}^{-}=-\left(H_{3}^{+}\right)^{*}, H_{3 M}^{-}=$ $-\left(H_{3 M}^{+}\right)^{*}, H_{3}^{0}=-\left(H_{3}^{0}\right)^{*}$ and $H_{3}^{0}=-\left(H_{3}^{0}\right)^{*}$. The masses of these physical scalars can be obtained according to Eq. (5.3.12). Since the potential preserves the $S U(2)_{D}$ custodial symmetry, members of the physical scalar multiplets have degenerate masses. These masses are

$$
\begin{aligned}
& m_{5}^{2}=3\left(\lambda_{5} c_{M}^{2}+\lambda_{8} s_{M}^{2}\right) v^{2}, \\
& m_{3}^{2}=\lambda_{5} v^{2}, \quad m_{3 M}^{2}=\lambda_{5}\left(1+c_{M}^{2}\right) v^{2},
\end{aligned}
$$


In general, the $H_{1}^{0}, H_{1 M}^{0}$ and $H_{1}^{0 \prime}$ can mix through the mass-squared matrix

$$
\mathcal{M}_{\text {singlets }}^{2}=v^{2}\left(\begin{array}{ccc}
8 s_{2}^{2}\left(\lambda_{1}+\lambda_{4}\right) & 8 s_{2} s_{2 M} \lambda_{4} & 2 \sqrt{6} s_{2} s_{M} \lambda_{4} \\
8 s_{2} s_{2 M} \lambda_{4} & 8 s_{2 M}^{2}\left(\lambda_{2}+\lambda_{4}\right) & 2 \sqrt{6} s_{2 M} s_{M} \lambda_{4} \\
2 \sqrt{6} s_{2} s_{M} \lambda_{4} & 2 \sqrt{6} s_{2 M} s_{M} \lambda_{4} & 3 s_{M}^{2}\left(\lambda_{3}+\lambda_{4}\right)
\end{array}\right)
$$

Hence, the mass eigenstates of the mixing of the $H_{1}^{0}, H_{1 M}^{0}$ and $H_{1}^{0 \prime}$ can be achieved through

$$
\left(\begin{array}{c}
\widetilde{H} \\
\widetilde{H}^{\prime} \\
\widetilde{H}^{\prime \prime}
\end{array}\right)=\left(\begin{array}{ccc}
a_{1,1} & a_{1,1 M} & a_{1,1^{\prime}} \\
a_{1 M, 1} & a_{1 M, 1 M} & a_{1 M, 1^{\prime}} \\
a_{1^{\prime}, 1} & a_{1^{\prime}, 1 M} & a_{1^{\prime}, 1^{\prime}}
\end{array}\right)\left(\begin{array}{c}
H_{1}^{0} \\
H_{1 M}^{0} \\
H_{1}^{0 \prime}
\end{array}\right)
$$

By convention, the $\widetilde{H}, \widetilde{H}^{\prime}, \widetilde{H}^{\prime \prime}$ have their masses in increasing order. The lightest one, $\widetilde{H}$ plays the role of the candidate for the $125 \mathrm{GeV}$ SM-like Higgs boson in the EW $\nu_{R}$ model. Note that, the mixing will disappear if $\lambda_{4}=0$. Due to the complexity of the parameter space of the $\mathcal{M}_{\text {singlets }}$, we have to diagonalize the mass matrix in Eq. (5.3.21) numerically.

\subsection{The 125 GeV Higgs Boson Candidate}

At the current stage, the measured signal strength of the $125 \mathrm{GeV}$ Higgs boson at the LHC is very close to the prediction of the Standard Model as we can see in Fig. 5.1. It is required of any BSM model to have a scalar with the mass in the region of $125 \mathrm{GeV}$ and the signal strength to agree with the measurement from the LHC. Finding the $125 \mathrm{GeV}$ Higgs boson candidate of the $\mathrm{EW} \nu_{R}$ model is a two-step procedure.

- Diagonalize the mass matrix in Eq. (5.3.21) numerically to find the solutions that have the lowest eigenvalue of $125 \mathrm{GeV}$ which is corresponding to the mass of $\widetilde{H}$. Note that, in our work, we allow the mass of $\widetilde{H}$ to vary in the region of $125.7 \pm 1.0 \mathrm{GeV}$ which covers well the mass of $125 \mathrm{GeV}$ SM-like Higgs boson measured at the LHC most recently [27]. 
- With the parameter space taken from the first step, we calculate the signal strength of the $125 \mathrm{GeV}$ Higgs boson in the model and find all the cases which satisfy the signal strength of the $125 \mathrm{GeV}$ SM-like Higgs boson measured at ATLAS/CMS. Because this steps was done before the combined result of ATLAS and CMS, so we just considered the value of the measured signal strength of CMS [30, 31, 32, 33] as the reference for our numerical calculation.

\subsubsection{The Decay The $125 \mathrm{GeV}$ Higgs Boson Candidate}

We devote this section to present the key characteristics of the candidate of the $\mathrm{EW} \nu_{R}$ model for the $125 \mathrm{GeV}$ Higgs boson. The candidate here is $\widetilde{H}$ which is the lightest state of the three CP-even states, $H_{1}^{0}, H_{1 M}^{0}, H_{1}^{0 \prime}$.

As shown in Eq. (5.3.19), $H_{1}^{0}$ is made from the real part of the neutral component of the scalar doublet $\Phi_{2}$ which only couples to the SM fermions. One may say that this $\Phi_{2}$ and then $H_{1}^{0}$ behave like the Standard Model scalar doublet. On the other hand, $H_{1 M}^{0}$ is constructed from the real part of the neutral component of the newly added scalar doublet $\Phi_{2 M}$ which only couples to the mirror fermions. Then $H_{1 M}^{0}$ only couples to the mirror fermions. While $H_{1}^{0 \prime}$ comes purely from the scalar triplets $\chi$, thus it does not couple to both the SM fermions and the mirror fermions.

Analytically, the lightest mixing state of these three singlets can be expressed as in Eq. (5.3.22):

$$
\widetilde{H}=a_{1,1} H_{1}^{0}+a_{1,1 M} H_{1 M}^{0}+a_{1,1^{\prime}} H_{1}^{0 \prime}
$$

As the result, the decay property of the $\widetilde{H}$ depends on the decay modes of the $H_{1}^{0}$, $H_{1 M}^{0}$ and $H_{1}^{0 \prime}$ and the mixing matrix between these three singlets. We consider all the kinematically allowed channels among $W^{+} W^{-}, Z Z, \gamma \gamma, g g, b \bar{b}, \tau \bar{\tau}$. The decay of the SM Higgs is calculated up to the next-leading order in QCD [34]. In general, the decay of a neutral CP-even Higgs can be calculated by simply rescaling the 
corresponding decay modes of the SM Higgs.

- The $\widetilde{H} \rightarrow g g$ channel.

In the Standard Model, the decay $H_{S M}^{0} \rightarrow g g$ goes mostly through the triangle loop of the top quark because it is the heaviest one among the SM quarks. The partial width of this process is given by

$$
\Gamma^{S M}\left(H_{S M}^{0} \rightarrow g g\right)=\frac{\alpha_{S}^{2} g^{2} m_{H_{S M}^{0}}^{3}}{128 \pi^{3} M_{W}^{2}}\left|\sum_{i} \frac{1}{2 v_{H^{0}} / v} F_{1 / 2}\left(\tau_{i}\right)\right|^{2}
$$

where, for a loop of quark having mass $m_{i}, \tau_{i}=4 m_{i}^{2} / m_{H_{S M}^{0}}^{2}[16]$, and the common function $F_{1 / 2}(\tau)$ is given by

$$
\begin{aligned}
& F_{1 / 2}(\tau)=-2 \tau[1+(1-\tau) f(\tau)] . \\
& f(\tau)=\left[\sin ^{-1}(1 / \sqrt{\tau})\right]^{2}, \quad \text { if } \tau \geq 1, \\
& =\frac{1}{4}\left[\log \left(\eta_{+} / \eta_{-}\right)-\imath \pi\right]^{2}, \quad \text { if } \tau<1 \text {; }
\end{aligned}
$$

here,

$$
\eta_{ \pm} \equiv(1 \pm \sqrt{1-\tau})
$$

While in the $\mathrm{EW} \nu_{R}$ model, besides top quark, 6 mirror quarks also have their contributions to the loop. By rescaling, we have

$$
\begin{aligned}
\Gamma^{E W \nu_{R}}(\widetilde{H} \rightarrow g g)= & \Gamma^{S M}\left(H_{S M}^{0} \rightarrow g g\right) \times \frac{1}{\left|F_{1 / 2}\left(\tau_{\text {top }}\right)\right|^{2}} \\
& \times\left|\frac{a_{1,1}}{s_{2}} F_{1 / 2}\left(\tau_{\text {top }}\right)+\frac{a_{1,1 M}}{s_{2 M}} \sum_{i} F_{1 / 2}\left(\tau_{M F_{i}}\right)\right|^{2}
\end{aligned}
$$

$\sum_{i}$ is taken over all the mirror quarks; $a_{1,1}, a_{1,1 M}$ are the mixing coefficients of $H_{1}^{0}, H_{1 M}^{0}$ in $\widetilde{H}$, respectively. Note that $H_{1}^{0 \prime}$ does not couple to both the SM and the mirror quarks. $\Gamma^{S M}$ is calculated up to the next-leading order in QCD [34].

- The $\widetilde{H} \rightarrow \gamma \gamma$ channel. 
Generally, $H^{0} \rightarrow \gamma \gamma$ at the loop level through the loops of both fermions, vector bosons, and additional scalars. For a general BSM Lagrangian such as

$$
\mathcal{L}_{i n t}=\frac{-m_{f}}{v_{H^{0}}} \bar{\psi} \psi H^{0}+g M_{W} \lambda_{W} W_{\mu}^{+} W^{\mu-} H^{0}+\frac{g \lambda_{S}}{M_{W}} S^{+} S^{-} H^{0}
$$

where $v_{H^{0}}$ is the vacuum expectation value of $H^{0}, v=2 M_{W} / g \sim \sqrt{\sum_{\text {all } H^{0}{ }_{\mathrm{s}} v_{H^{0}}^{2}}}$, $\psi$ is a fermion of mass $m_{f}, S^{ \pm}$is a charged BSM scalar. The partial decay width of $H^{0} \rightarrow \gamma \gamma$ is given by [16]:

$$
\Gamma\left(H^{0} \rightarrow \gamma \gamma\right)=\frac{\alpha^{2} g^{2}}{1024 \pi^{3}} \frac{m_{H^{0}}^{3}}{M_{W}^{2}}\left|\sum_{i} N_{c i} Q_{i}^{2} F_{s}\left(\tau_{i}\right)\right|^{2} .
$$

Here, $\sum_{i}$ is performed over all the particles of spin- $s ; s=$ spin- 0 , spin- $1 / 2$, and spin-1 are the spins; $Q_{i}$ is the electric charge in the unit of $e$. The common function $F_{s}\left(\tau_{i}\right)$ is given by

$$
\begin{aligned}
F_{1}(\tau) & =\lambda_{W} \tau[3+(4-3 \tau) f(\tau)], \\
F_{1 / 2}(\tau) & =-2 \tau[1+(1-\tau) f(\tau)], \\
F_{0}(\tau) & =2 \lambda_{S}[1-\tau f(\tau)],
\end{aligned}
$$

with $\tau=4 m_{i}^{2} / m_{H^{0}}^{2}$ and $f(\tau)$ is given by Eq (5.4.4).

In calculating $\Gamma^{E W \nu_{R}}(\widetilde{H} \rightarrow \gamma \gamma)$, we define the amplitude of $H^{0} \rightarrow \gamma \gamma$ as

$$
\mathcal{A}\left(H^{0} \rightarrow \gamma \gamma\right)=\sqrt{\frac{\alpha^{2} g^{2}}{1024 \pi^{3}} \frac{m_{H^{0}}^{3}}{M_{W}^{2}}}\left(\sum_{i} N_{c i} Q_{i}^{2} F_{s}\left(\tau_{i}\right)\right)
$$

From Eq. (5.3.22), we have

$$
\begin{aligned}
\Gamma^{E W \nu_{R}}(\widetilde{H} \rightarrow \gamma \gamma)= & \mid a_{1,1} \mathcal{A}^{E W \nu_{R}}\left(H_{1}^{0} \rightarrow \gamma \gamma\right)+a_{1,1 M} \mathcal{A}^{E W \nu_{R}}\left(H_{1 M}^{0} \rightarrow \gamma \gamma\right) \\
& +\left.a_{1,1^{\prime}} \mathcal{A}^{E W \nu_{R}}\left(H_{1}^{0 \prime} \rightarrow \gamma \gamma\right)\right|^{2}
\end{aligned}
$$

where $a_{1,1}, a_{1,1 M}, a_{1,1^{\prime}}$ are the mixing coefficients of $H_{1}^{0}, H_{1 M}^{0}$ and $H_{1}^{0 \prime}$ in $\widetilde{H}$ mass eigenstate, respectively. These are the elements in the first row of the mixing matrix $\left\{a_{i, j}\right\}$ in $\operatorname{Eq}(5.3 .22)$. 
The $W^{ \pm}, G_{3}^{ \pm}$loops and the scalar loops of $H_{3}^{ \pm}, H_{3 M}^{ \pm}, H_{5}^{ \pm}$and $H_{5}^{ \pm \pm}$contribute to $\mathcal{A}^{E W \nu_{R}}\left(H_{1}^{0} \rightarrow \gamma \gamma\right), \mathcal{A}^{E W \nu_{R}}\left(H_{1 M}^{0} \rightarrow \gamma \gamma\right)$ as well as $\mathcal{A}^{E W \nu_{R}}\left(H_{1}^{0 \prime} \rightarrow \gamma \gamma\right)$. The SM fermion loops contribute only to $\mathcal{A}^{E W \nu_{R}}\left(H_{1}^{0} \rightarrow \gamma \gamma\right)$, while the charged mirror fermion loops contribute only to $\mathcal{A}^{E W \nu_{R}}\left(H_{1 M}^{0} \rightarrow \gamma \gamma\right)$. No fermion loop contributes to $\mathcal{A}^{E W \nu_{R}}\left(H_{1}^{0 \prime} \rightarrow \gamma \gamma\right)$.

- The $\widetilde{H} \rightarrow W W / Z Z$ channels.

$H_{1}^{0}, H_{1 M}^{0}, H_{1}^{0 \prime}$ all couple to $W / Z$ with the coupling coefficients of $s_{2}, s_{2 M}, 2 \frac{\sqrt{2}}{\sqrt{3}} s_{M}$ defined in Eq. (5.3.11), respectively. By rescaling, we have

$$
\begin{aligned}
\Gamma^{E W \nu_{R}}(\widetilde{H} \rightarrow W W, Z Z)= & \Gamma^{S M}\left(H_{S M}^{0} \rightarrow W W, Z Z\right) \\
& \left|a_{1,1} s_{2}+a_{1,1 M} s_{2 M}+a_{1,1^{\prime}} \frac{2 \sqrt{2}}{\sqrt{3}} s_{M}\right|^{2}
\end{aligned}
$$

- The $\widetilde{H} \rightarrow f \bar{f}$ channels.

At the mass of $125 \mathrm{GeV}, \widetilde{H}$ only decays into the light SM fermions at tree level, such as $\widetilde{H} \rightarrow \tau \bar{\tau}, b \bar{b}$. Among $H_{1}^{0}, H_{1 M}^{0}, H_{1}^{0 \prime}$, only $H_{1}^{0}$ couples to these light SM fermions. Hence, for $\widetilde{H}$ with the mass of $125 \mathrm{GeV}$, we have

$$
\Gamma^{E W \nu_{R}}(\widetilde{H} \rightarrow f \bar{f})=\Gamma^{S M}\left(H^{0} \rightarrow f \bar{f}\right) \times\left|\frac{a_{1,1}}{s_{2}}\right|^{2}
$$

- The total width of $\widetilde{H}$.

In conclusion, the total width of $\widetilde{H}$ is calculated by simply adding all of these partial widths:

$$
\begin{aligned}
\Gamma_{\widetilde{H}}= & \Gamma_{\widetilde{H} \rightarrow b \bar{b}}+\Gamma_{\widetilde{H} \rightarrow \tau \bar{\tau}}+\Gamma_{\widetilde{H} \rightarrow c \bar{c}}+\Gamma_{\widetilde{H} \rightarrow W^{+} W^{-}} \\
& +\Gamma_{\widetilde{H} \rightarrow Z Z}+\Gamma_{\widetilde{H} \rightarrow g g}+\Gamma_{\widetilde{H} \rightarrow \gamma \gamma} .
\end{aligned}
$$

\subsubsection{The Signal Strength of The $125 \mathrm{GeV}$ Higgs Boson Candidate}

At the LHC, the discovery of the $125 \mathrm{GeV}$ Higgs boson is realized through its signal strength in 6 channels, $W^{+} W^{-}, Z Z, \gamma \gamma, g g, b \bar{b}, \tau \bar{\tau}$, which is defined in Eq. 
(5.6.2). In this definition, the product of the production cross section through the initial state and the branching ratio of the Higgs boson in each channel is calculated by [34]

$$
\sigma . B R(i i \rightarrow H \rightarrow X X)=\sigma_{i i} \times B R(H \rightarrow X X)
$$

where $\sigma_{i i}$ is the production cross section through the initial state $i i$. While the branching ratio of the decay mode $H \rightarrow X X$ is

$$
B R(H \rightarrow X X)=\frac{\Gamma(H \rightarrow X X)}{\Gamma_{H}}
$$

here $\Gamma(H \rightarrow X X)$ is the partial width of the decay channel; $\Gamma_{H}$ is the total width of the Higgs. The observed data is presented in terms of the signal strength given by

$$
\mu_{\text {data }}=\frac{\sigma \cdot B R(H \rightarrow X X)_{d a t a}}{\sigma \cdot B R\left(H_{S M} \rightarrow X X\right)}
$$

Hence, we define the signal strength of the $125 \mathrm{GeV}$ Higgs boson candidate in the $\mathrm{EW} \nu_{R}$ model as

$$
\mu_{E W \nu_{R}}=\frac{\sigma \cdot B R(\widetilde{H} \rightarrow X X)}{\sigma \cdot B R\left(H_{S M} \rightarrow X X\right)}
$$

Then we compare $\mu_{E W \nu_{R}}$ with $\mu_{\text {data }}$ in each decay channel. In principle, the 125 GeV Higgs particle is produced through four processes: gluon fusion, vector boson fusion, $W H / Z H$ Higgs associated production, $t t H$ associated Higgs production. At the LHC, the production of the $125 \mathrm{GeV}$ Higgs particle is dominated by the gluon fusion process. Hence, we just compare the $\mu^{E W \nu_{R}}$ with $\mu^{\text {data }}$ in the gluon fusion production mode. We calculate the signal strength at a given mass of Higgs particle as

$$
\mu_{E W \nu_{R}}=\frac{\sigma_{\mathrm{EW} \nu_{R}}(g g \rightarrow \widetilde{H}) \times B R(\widetilde{H} \rightarrow X X)}{\sigma_{S M}\left(g g \rightarrow H_{S M}\right) \times B R\left(H_{S M} \rightarrow X X\right)}
$$


By using the effective coupling method, the ratio of the production cross section is

$$
\frac{\sigma_{\mathrm{EW} \nu_{R}}(g g \rightarrow \widetilde{H})}{\sigma_{S M}\left(g g \rightarrow H_{S M}\right)}=\frac{\Gamma_{\mathrm{EW} \nu_{R}}(\widetilde{H} \rightarrow g g)}{\Gamma_{S M}\left(H_{S M} \rightarrow g g\right)} .
$$

From Eq. (5.4.6), we have

$$
\frac{\sigma_{\mathrm{EW} \nu_{R}}(g g \rightarrow \widetilde{H})}{\sigma_{S M}\left(g g \rightarrow H_{S M}\right)}=\frac{1}{\left|F_{1 / 2}\left(\tau_{\text {top }}\right)\right|^{2}}\left|\frac{a_{1,1}}{s_{2}} F_{1 / 2}\left(\tau_{\text {top }}\right)+\frac{a_{1,1 M}}{s_{2 M}} \sum_{i} F_{1 / 2}\left(\tau_{M F_{i}}\right)\right|^{2}
$$

The branching ratios of the $H_{S M} \rightarrow W^{+} W^{-}, Z Z, \gamma \gamma, g g, b \bar{b}, \tau \bar{\tau}$ decay processes are calculated by the Higgs Working Group up to next-to-leading order of QCD [34]. While the branching ratios of the corresponding channels of $\widetilde{H}$ are calculated by

$$
B R(\widetilde{H} \rightarrow X X)=\frac{\Gamma(\widetilde{H} \rightarrow X X)}{\Gamma_{\widetilde{H}}},
$$

where $X X=W^{+} W^{-}, Z Z, \gamma \gamma, g g, b \bar{b}, \tau \bar{\tau}$. The partial widths and the total width

of $\widetilde{H}$ are given by Eqs. (5.4.6, 5.4.11, 5.4.12, 5.4.13). From Eqs. (5.4.12, 5.4.13), one has

$$
\begin{aligned}
\frac{\Gamma^{E W \nu_{R}}\left(\widetilde{H} \rightarrow W^{+} W^{-}\right)}{\Gamma^{S M}\left(H_{S M}^{0} \rightarrow W^{+} W^{-}\right)} & =\frac{\Gamma^{E W \nu_{R}}(\widetilde{H} \rightarrow Z Z)}{\Gamma^{S M}\left(H_{S M}^{0} \rightarrow Z Z\right)}, \\
\frac{\Gamma^{E W \nu_{R}}(\widetilde{H} \rightarrow b \bar{b})}{\Gamma^{S M}\left(H_{S M}^{0} \rightarrow b \bar{b}\right)} & =\frac{\Gamma^{E W \nu_{R}}(\widetilde{H} \rightarrow \tau \bar{\tau})}{\Gamma^{S M}\left(H_{S M}^{0} \rightarrow \tau \bar{\tau}\right)}
\end{aligned}
$$

Consequently, the signal strengths of $\widetilde{H} \rightarrow W^{+} W^{-}$and $\widetilde{H} \rightarrow Z Z$ are equal. Similarly, the signal strengths of $\widetilde{H} \rightarrow b \bar{b}$ and $\widetilde{H} \rightarrow \tau \bar{\tau}$ are the same.

\subsubsection{The parameter space}

In this section, we discuss the limit of the parameter space in the analysis about the signal strength of the $125 \mathrm{GeV}$ SM-like Higgs boson candidate of the EW $\nu_{R}$ model. In numerical calculation, the parameter space consists twelve masses of the right-handed neutrinos and charged mirror fermions, ten masses of the new 
scalars, three mixing angles in the scalar sector, and six coupling constants in the scalar potential.

- The limit of the masses of the right-handed neutrinos.

As discussed in the part 4.5.1, the right-handed neutrino masses must satisfy the condition: $M_{R} \geq M_{Z} / 2$ to avoid the effect on the Z-width. The upper limit is set at $300 \mathrm{GeV}$ based on the perturbative limit of the theory. However, this constraint can be relaxed so that the $M_{R}$ can be higher $300 \mathrm{GeV}$.

- The limit of the masses of the charged mirror fermions.

The lower limit of the masses of the charged mirror leptons is $102 \mathrm{GeV}$ based on the search for heavy charged leptons and quarks at LEP3 [23]. Note that in this search, LEP3 was looking for the heavy charged fermions in the sequential decay processes such as $Q^{\prime} \rightarrow b Z \rightarrow b l \bar{l}, b q \bar{q}$, etc. However, the decay of the charged mirror fermions of the $\mathrm{EW} \nu_{R}$ model is very different. They only couple to the Standard Model counterparts through the scalar singlet $\Phi_{S}$ as seen in the section 3.2. The charged mirror fermions decay into the SM fermions through processes such as $q^{M} \rightarrow q \Phi_{S}, l^{M} \rightarrow l \Phi_{S}$. $\Phi_{S}$ turns out to be a missing energy in these decay processes. Therefore, the signal of these processes differ from the sequential processes in the search of LEP3 because of the appearance of the displaced vertices.

Moreover, the length of the decay of $q^{M} \rightarrow q \Phi_{S}, l^{M} \rightarrow l \Phi_{S}$ depends on the Yukawa coupling of $\Phi_{S} \bar{f} f^{M}$. If the decay length is beyond few centimeters, then the displaced vertices appear inside of the silicon vertex detector. Hence, the constraint from the LEP3 searches does not directly apply on the charged mirror fermions in the model.

- The limit of the mass of the scalars, the Yukawa couplings, and the VEVs.

The lower limit of the mass of the scalars is $M_{Z}$ as the same as in the part 4.5.1. We also impose the perturbative constraint of the theory by requiring $\alpha_{f}=$ $g_{f}^{2} / 4 \pi \leq O(1)$ for the Yukawa couplings of the mirror fermions and $\lambda_{i} / 4 \pi \leq O(1)$ 
in the scalar sector. Three mixing angles in the scalar sector, $s_{2}, s_{2 M}, s_{M}$, depend on the VEVs of the scalar fields $\Phi_{2}, \Phi_{2 M}, \tilde{\chi}$, respectively. They are tied up by the identity $v_{2}^{2}+v_{2 M}^{2}+8 v_{M}^{2}=(246 \mathrm{GeV})^{2}$. Besides, these VEVs also appear in the masses of the mirror fermions. Therefore, they are also constrained by the perturbative constraint of the theory.

For instance, if we put the constraint $g_{f}^{2} / 4 \pi \leq 1.5$ on the Yukawa couplings of the mirror charged fermions, the upper limit of their masses can be $m_{M F} \leq$ $715 \mathrm{GeV}$. While we limit $\lambda_{i} \leq 5.2 \pi$ for the scalar coupling. Thus the allowed ranges of the VEVs of the scalar fields and so for the mixing angles in the scalar sector are given by Table 5.1 
Table 5.1. Allowed ranges of VEVs and parameters defined in Eq. (5.3.11). All values are given in $\mathrm{GeV}$.

\begin{tabular}{|l|r|}
\hline $69 \lesssim v_{2} \lesssim 241$ & $0.28 \lesssim s_{2} \lesssim 0.98$ \\
$33 \lesssim v_{2 M} \lesssim 233$ & $0.13 \lesssim s_{2 M} \lesssim 0.95$ \\
$13 \lesssim v_{M} \lesssim 83$ & $0.15 \lesssim s_{M} \lesssim 0.95$ \\
\hline
\end{tabular}

\subsection{The Dual Nature of the $125 \mathrm{GeV}$ SM-like Candidate of The $\mathrm{EW} \nu_{R}$ Model}

The first step in the numerical calculation is to diagonalize the mass matrix in Eq. (5.3.21) in the limit of the parameter space listed in the section 5.4.3. Under the requirement of the lightest mass eigenstate of $125.7 \pm 1.0 \mathrm{GeV}$, four million combinations were found. This lightest one, $\widetilde{H}$, is the candidate for the $125 \mathrm{GeV}$ SM-like Higgs boson of the $\mathrm{EW} \nu_{R}$ model.

The next step is to calculate the signal strengths of $\widetilde{H} \rightarrow W^{+} W^{-}, Z Z, \gamma \gamma, g g$, $b \bar{b}, \tau \bar{\tau}$ as Eq. (5.4.19). We varied $0 \leq \lambda_{5} \leq 15$, with the step of $\Delta \lambda_{5} \sim 1.07$. To reduce the calculating time, we fixed several parameters

$$
\begin{aligned}
\lambda_{8} & =-1, m_{H_{3}^{+}}=600 \mathrm{GeV}, m_{H_{3 M}^{+}}=700 \mathrm{GeV} \\
m_{H_{5}^{+}} & =200 \mathrm{GeV}, m_{H_{5}^{++}}=320 \mathrm{GeV}, m_{q_{3}^{M}}=120 \mathrm{GeV} \\
m_{q_{1}^{M}} & =m_{q_{2}^{M}}=m_{l^{M}}=102 \mathrm{GeV} .
\end{aligned}
$$

When we carried out this analysis, the signal strength measurements of the 125 GeV SM-like Higgs boson from the reports of ATLAS and CMS neither agreed within $1 \sigma$ nor published the combined result. Therefore, we compared the predic- 
tion of the signal strength of the $\widetilde{H}$ at $125 \mathrm{GeV}$ in the $\mathrm{EW} \nu_{R}$ model to the results of CMS without any prejudice.

As the result, we got 1501 out of 4 million combinations found in the first step which produce the signal strength of $\widetilde{H} \rightarrow W^{+} W^{-}, Z Z, \gamma \gamma, g g, b \bar{b}, \tau \bar{\tau}$ within $1 \sigma$ constraints from the CMS measurements. Among these 1501 combinations, there are two distinguishable scenarios depending on how much the $H_{1}^{0}$, which is made from the Standard Model like doublet $\Phi_{2}$, contributes to $\widetilde{H}$. We call the two scenarios as Dr.Jekyll and Mr.Hyde literally.

In the case of Dr.Jekyll, $H_{1}^{0}$ is the dominant component of the mixing state $\widetilde{H}$. This is the case where $\widetilde{H}$ is very like the Standard Model Higgs boson. While in the $M r . H y d e$ case, $H_{1}^{0}$ is the sub-dominant component of $\widetilde{H}$ which means $\widetilde{H}$ is very different with the Standard Model Higgs boson. Again, both of these cases have the signal strength satisfying the constraint from CMS measurements. This is the unique characteristic of the $\mathrm{EW} \nu_{R}$ model. Here we present several cases to illustrate our results in the Figure 5.2. 


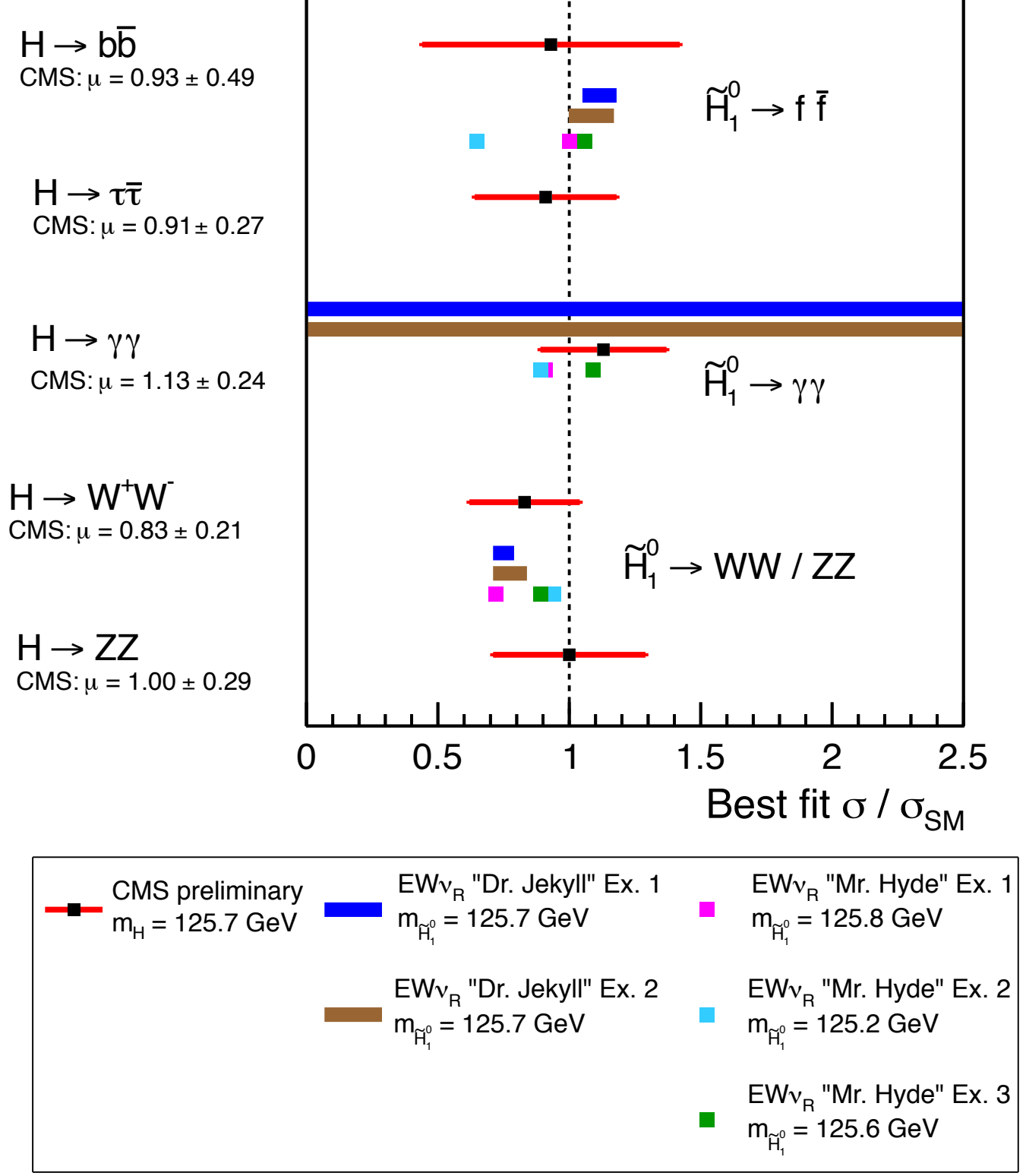

Figure 5.2. Figure shows the predictions of $\mu\left(\widetilde{H} \rightarrow b \bar{b}, \tau \bar{\tau}, \gamma \gamma, W^{+} W^{-}, Z Z\right)$ in the EW $\nu_{R}$ model for Examples 1 and 2 in Dr. Jekyll and Examples 1,2 and 3 in $M r$. Hyde scenarios, in comparison with corresponding best fit values by CMS [30, 31, 32, 33]. 
As defined in Eqs. (5.1.1, 5.4.19), the signal strength is the product of the production cross section and the branching ratio of the Higgs boson normalized by the same product of the SM. Looking for the candidate of the $125 \mathrm{GeV}$ SM-like Higgs boson is to find all the cases when $\widetilde{H}$ has the production cross section times the branching ratio satisfying the $1 \sigma$ constraint of CMS. The likely scenario is when both the production cross section and the branching ratio of $\widetilde{H}$ are similar to those of the Standard Model Higgs boson. Interestingly, there is also a different scenario in which both the production cross section and the branching ratio of $\widetilde{H}$ are very different with the predictions of the Standard Model, but their product, the signal strength, is still in agreement with the experimental measurements. We present these scenarios in details in next section.

\subsubsection{The SM-like $H_{1}^{0}$ is The Dominant Component of $\widetilde{H}$}

In the case of Dr.Jekyll as illustrated by two examples Ex. 1 and Ex. 2, $\widetilde{H}$ is dominant by the SM-like $H_{1}^{0}$. In fact, while scanning the parameter space to have the signal strength of $\widetilde{H}$ in agreement with the CMS's results, the coefficient of $H_{1}^{0}$ in Eq. (5.4.1) $s_{2} \geq 0.9$ to satisfy the constraint.

In Example 1, the set of parameters is : $\lambda_{1}=-0.077, \lambda_{2}=14.06, \lambda_{3}=$ 15.4, $\lambda_{4}=0.1175, \lambda_{5}=15, \lambda_{8}=-1$ and $m_{H_{5}^{+}}=500 \mathrm{GeV}, m_{H_{5}^{++}}=540 \mathrm{GeV}$. Fixing these along with $s_{2}=0.92, s_{2 M}=0.16, s_{M} \approx 0.36$, the mixing matrix is given by:

$$
\left(\begin{array}{c}
\widetilde{H} \\
\widetilde{H}^{\prime} \\
\widetilde{H}^{\prime \prime}
\end{array}\right)=\left(\begin{array}{ccc}
0.998 & -0.0518 & -0.0329 \\
0.0514 & 0.999 & -0.0140 \\
0.0336 & 0.0123 & 0.999
\end{array}\right)\left(\begin{array}{c}
H_{1}^{0} \\
H_{1 M}^{0} \\
H_{1}^{0 \prime}
\end{array}\right) .
$$

We can see that $\widetilde{H} \sim H_{1}^{0}, \widetilde{H}^{\prime} \sim H_{1 M}^{0}, \widetilde{H}^{\prime \prime} \sim H_{1}^{0 \prime}$. The corresponding eigenvalues are $m_{\widetilde{H}}=125.7 \mathrm{GeV}, m_{\widetilde{H}^{\prime}}=420 \mathrm{GeV}, m_{\widetilde{H}^{\prime \prime}}=601 \mathrm{GeV}$. We vary $a_{1,1 M}$ - the $(1,2)$ element of the $3 \times 3$ matrix between $(-0.0515,-0.05295)$, the the signal strength of $\widetilde{H}$ still satisfy CMS constraints. 
Similarly, in Example 2, the corresponding parameter set is $\lambda_{1}=0.0329, \lambda_{2}=$ $14.2, \lambda_{3}=15.4, \lambda_{4}=0.0056, \lambda_{5}=15, \lambda_{8}=-1$, and $m_{H_{5}^{+}}=590 \mathrm{GeV}, m_{H_{5}^{++}}=$ $600 \mathrm{GeV}$. Then the mass eigenstates are determined by

$$
\left(\begin{array}{c}
\widetilde{H} \\
\widetilde{H}^{\prime} \\
\widetilde{H}^{\prime \prime}
\end{array}\right)=\left(\begin{array}{ccc}
0.99999 \ldots & -2.49 \times 10^{-3} & -1.60 \times 10^{-3} \\
2.49 \times 10^{-3} & 0.99999 \ldots & -5.30 \times 10^{-4} \\
1.60 \times 10^{-3} & 5.26 \times 10^{-4} & 0.99999 . .
\end{array}\right)\left(\begin{array}{l}
H_{1}^{0} \\
H_{1 M}^{0} \\
H_{1}^{0 \prime}
\end{array}\right)
$$

Still, we have $\widetilde{H} \sim H_{1}^{0}, \widetilde{H}^{\prime} \sim H_{1 M}^{0}, \widetilde{H}^{\prime \prime} \sim H_{1}^{0 \prime}$ and $m_{\widetilde{H}}=125.7 \mathrm{GeV}, m_{\widetilde{H}^{\prime}}=$ $420 \mathrm{GeV}, m_{\widetilde{H}^{\prime \prime}}=599 \mathrm{GeV}$. The allowed range for $a_{1,1 M}$ - the $(1,2)$ element of the $3 \times 3$ matrix is $(-1.20,-3.40) \times 10^{-3}$.

In this class of examples, $\widetilde{H}$ behaves just like the Standard Model Higgs boson in terms of coupling to other particles. Therefore, we could expect that the production cross section and the branching ratio of $\widetilde{H} \rightarrow b \bar{b}, \tau \bar{\tau}, \gamma \gamma, W^{+} W^{-}, Z Z$ are similar to those of the Standard Model Higgs boson. For example, in Example 2, one list explicit the partial widths, the branching ratios, and the ggF production cross-sections of the individual decay channels of $\widetilde{H}$ and $H_{S M}$ in the Table. 5.2 
Table 5.2. In the ggF production mode of the Higgs boson, partial widths and branching ratios for various channels in $\mathrm{SM}$ (for $m_{H_{S M}}=125.7 \mathrm{GeV}$ ) with total width $=4.17 \mathrm{E}-3 \mathrm{GeV}$, and the $\mathrm{EW} \nu_{R}$ model for Dr. Jekyll Example 2 scenario: $a_{1,1 M}=-0.0025$, where $m_{\widetilde{H}}=125.7 \mathrm{GeV}$, total width $=4.45 \mathrm{E}-3$ $\mathrm{GeV}$ and $\widetilde{H} \sim H_{1}^{0}$. All the partial widths are given in $\mathrm{GeV}$.

\begin{tabular}{|c|c|c|c|c|c|c|c|}
\hline & \multicolumn{3}{|c|}{ SM } & \multicolumn{3}{|c|}{$\mathrm{EW} \nu_{R}$} & \multirow{2}{*}{$\mu$} \\
\hline & $\begin{array}{c}\Gamma_{H \rightarrow g g} \\
\propto \sigma_{g g \rightarrow H}\end{array}$ & $\Gamma$ & $\mathrm{BR}$ & $\begin{aligned} & \Gamma_{\widetilde{H} \rightarrow g g} \\
\propto & \sigma_{g g \rightarrow H}\end{aligned}$ & $\Gamma$ & $\mathrm{BR}$ & \\
\hline$\widetilde{H} \rightarrow W^{+} W^{-}$ & $3.55 \mathrm{E}-04$ & $9.42 \mathrm{E}-04$ & $2.26 \mathrm{E}-01$ & $3.46 \mathrm{E}-04$ & 7.63E-04 & $1.72 \mathrm{E}-01$ & 0.74 \\
\hline$\widetilde{H} \rightarrow Z Z$ & $3.55 \mathrm{E}-04$ & $1.17 \mathrm{E}-04$ & $2.81 \mathrm{E}-02$ & $3.46 \mathrm{E}-04$ & $9.49 \mathrm{E}-05$ & 2.13E-02 & 0.74 \\
\hline$\widetilde{H} \rightarrow b \bar{b}$ & $3.55 \mathrm{E}-04$ & $2.36 \mathrm{E}-03$ & $5.66 \mathrm{E}-01$ & $3.46 \mathrm{E}-04$ & $2.79 \mathrm{E}-03$ & $6.26 \mathrm{E}-01$ & 1.07 \\
\hline$\widetilde{H} \rightarrow \tau \bar{\tau}$ & $3.55 \mathrm{E}-04$ & $2.59 \mathrm{E}-04$ & $6.21 \mathrm{E}-02$ & $3.46 \mathrm{E}-04$ & $3.06 \mathrm{E}-04$ & $6.87 \mathrm{E}-02$ & 1.07 \\
\hline$\widetilde{H} \rightarrow \gamma \gamma$ & $3.55 \mathrm{E}-04$ & $9.51 \mathrm{E}-06$ & $2.28 \mathrm{E}-03$ & $3.46 \mathrm{E}-04$ & $1.26 \mathrm{E}-05$ & $2.82 \mathrm{E}-03$ & 1.21 \\
\hline
\end{tabular}

\section{$\star$ Remarks}

- In Fig. 5.2, the signal strengths of the $\widetilde{H} \rightarrow \gamma \gamma$ channel of both Examples 1 and 2 are in a wide range. This happens because of the contribution of the charged scalars to the loop in the decay of $\widetilde{H} \rightarrow \gamma \gamma$. Particularly, we vary the mass of $\mathrm{H}_{5}^{+}, \mathrm{H}_{5}^{++}$in the range of $400-700 \mathrm{GeV}$. It means that there is always a set of parameters so that the signal strength of $\widetilde{H} \rightarrow \gamma \gamma$ satisfies the $1 \sigma$ experimental constraint.

- We allow the mixing parameter $a_{1,1 M}$ varying in the range of $(-0.0515,-0.05295)$ in Example 1 and $(-1.20--3.4) \times 10^{-3}$ in Example 2. As the result, we have two bands of the signal strength of $\widetilde{H} \rightarrow \gamma \gamma$ in each example. 
- The signal strengths of $\widetilde{H} \rightarrow W^{+} W^{-} / Z Z$ are the same as indicated in Eq. (5.4.23). Similarly, the signal strengths of $\widetilde{H} \rightarrow b \bar{b} / \tau \bar{\tau}$ are same as well. According to Eq. (5.4.12), $\mu(\widetilde{H} \rightarrow W W / Z Z) \propto \mid a_{1,1} s_{2}+a_{1,1 M} s_{2 M}+$ $\left.a_{1,1^{\prime}} \frac{2 \sqrt{2}}{\sqrt{3}} s_{M}\right|^{2}$. While $\mu(\widetilde{H} \rightarrow b \bar{b} / \tau \bar{\tau}) \propto\left|\frac{a_{1,1}}{s_{2}}\right|^{2}$ in Eq. (5.4.13). We can see that $\mu(\widetilde{H} \rightarrow W W / Z Z)$ and $\mu(\widetilde{H} \rightarrow b \bar{b} / \tau \bar{\tau})$ depend on $s_{2}$ in the opposite direction. As illustrated in Fig. 5.2, $\mu(\widetilde{H} \rightarrow b \bar{b} / \tau \bar{\tau})$ is greater the central value of the experimental result, while $\mu(\widetilde{H} \rightarrow W W / Z Z)$ is smaller than the central value of the corresponding experimental result.

- Finally, in Table 5.2, the detailed values of the ggF production cross section and the branching ratios of $\widetilde{H} \rightarrow b \bar{b}, \tau \bar{\tau}, \gamma \gamma, W^{+} W^{-}, Z Z$ are very close to these of the Standard Model Higgs boson. Naturally, the signal strengths of $\widetilde{H}$ decay in these channels are in the $1 \sigma$ experimental constraint of CMS.

\subsubsection{The SM-like $H_{1}^{0}$ is The Sub-dominant Component of $\widetilde{H}$}

One very special characteristic of the $\mathrm{EW} \nu_{R}$ is the existence of the cases in which the structure of the $125 \mathrm{GeV}$ SM-like candidate is very different with the SM Higgs boson but still has the signal strengths satisfying the experimental constraints as illustrated in the examples named $\mathrm{EW} \nu_{R}$ " $\mathrm{MrHyde}$ " Examples 1,2,3 in Fig. 5.2. It happens when the SM-like singlet $H_{1}^{0}$ has a small contribution to the mixing state $\widetilde{H}=a_{1,1} H_{1}^{0}+a_{1,1 M} H_{1 M}^{0}+a_{1,1^{\prime}} H_{1}^{0 \prime}$.

For instance, in $M r$.Hyde Ex. 1, the parameters are $s_{2}=0.900, s_{2 M}=0.270$, $s_{M}=0.341, \lambda_{1}=-0.481, \lambda_{2}=6.00, \lambda_{3}=1.46, \lambda_{4}=2.99, \quad \lambda_{5}=2, \quad \lambda_{8}=-1$. The eigenstates are given by

$$
\left(\begin{array}{c}
\widetilde{H} \\
\widetilde{H}^{\prime} \\
\widetilde{H}^{\prime \prime}
\end{array}\right)=\left(\begin{array}{ccc}
0.300 & -0.094 & -0.949 \\
0.334 & -0.921 & -0.197 \\
0.893 & 0.376 & 0.246
\end{array}\right)\left(\begin{array}{c}
H_{1}^{0} \\
H_{1 M}^{0} \\
H_{1}^{0 \prime}
\end{array}\right) .
$$


Thus, one has $\widetilde{H} \sim H_{1}^{0 \prime}, \widetilde{H}^{\prime} \sim H_{1 M}^{0}, \widetilde{H}^{\prime \prime} \sim H_{1}^{0} ; m_{\widetilde{H}}=125.8 \mathrm{GeV}, m_{\widetilde{H}^{\prime}}=416 \mathrm{GeV}$, $m_{\widetilde{H}^{\prime \prime}}=1100 \mathrm{GeV}, M_{R} \lesssim 105 \mathrm{GeV}$. The dominant component of $\widetilde{H}$ is $H_{1}^{0 \prime}$ which does not couple to neither the SM fermions nor the mirror fermions.

In $M r . H y d e$ Ex. 2, $s_{2}=0.514, s_{2 M}=0.841, s_{M}=0.168, \lambda_{1}=6.15, \lambda_{2}=$ 7.68, $\lambda_{3}=8.84, \lambda_{4}=-2.131502, \lambda_{5}=5, \lambda_{8}=-1$. The eigenstates are given by

$$
\left(\begin{array}{c}
\widetilde{H} \\
\widetilde{H}^{\prime} \\
\widetilde{H}^{\prime \prime}
\end{array}\right)=\left(\begin{array}{ccc}
0.188 & 0.091 & 0.978 \\
-0.941 & -0.268 & 0.207 \\
-0.281 & 0.959 & -0.035
\end{array}\right)\left(\begin{array}{l}
H_{1}^{0} \\
H_{1 M}^{0} \\
H_{1}^{0 \prime}
\end{array}\right)
$$

We still have $\widetilde{H} \sim H_{1}^{0 \prime}, \widetilde{H}^{\prime} \sim H_{1}^{0}, \widetilde{H}^{\prime \prime} \sim H_{1 M}^{0} ; m_{\widetilde{H}}=125.2 \mathrm{GeV}, m_{\widetilde{H}^{\prime}}=633 \mathrm{GeV}$, $m_{\widetilde{H}^{\prime \prime}}=1427 \mathrm{GeV}, M_{R} \lesssim 52.0 \mathrm{GeV}$.

In Mr.Hyde Ex. 3, another the parameters are $s_{2}=0.401, s_{2 M}=0.900$, $s_{M}=0.151, \lambda_{1}=4.76, \lambda_{2}=3.41, \lambda_{3}=7.71, \lambda_{4}=-1.29, \lambda_{5}=4, \lambda_{8}=-1$,

$$
\left(\begin{array}{c}
\widetilde{H} \\
\widetilde{H}^{\prime} \\
\widetilde{H}^{\prime \prime}
\end{array}\right)=\left(\begin{array}{ccc}
0.187 & 0.115 & 0.976 \\
0.922 & 0.321 & -0.215 \\
0.338 & -0.940 & 0.046
\end{array}\right)\left(\begin{array}{c}
H_{1}^{0} \\
H_{1 M}^{0} \\
H_{1}^{0 \prime}
\end{array}\right),
$$

with $\widetilde{H} \sim H_{1}^{0 \prime}, \widetilde{H}^{\prime} \sim H_{1}^{0}, \widetilde{H}^{\prime \prime} \sim H_{1 M}^{0} ; m_{\widetilde{H}}=125.6 \mathrm{GeV}, m_{\widetilde{H}^{\prime}}=454 \mathrm{GeV}$, $m_{\widetilde{H}^{\prime \prime}}=959 \mathrm{GeV}, M_{R} \lesssim 46.4 \mathrm{GeV}$. The detailed values of the ggF production cross section and the branching ratios are given in Table 5.3. 
Table 5.3. Partial width of $H \rightarrow g g$ as the measure of the production cross section, partial widths and branching ratios for various channels in SM (for $m_{H_{S M}}=125.6 \mathrm{GeV}$ and total width $4.15 \mathrm{E}-03 \mathrm{GeV}$ ), $\widetilde{H} \sim H_{1}^{0 \prime}\left(\right.$ with $m_{\widetilde{H}}=$ $125.6 \mathrm{GeV}$ and total width $1.34 \mathrm{E}-03 \mathrm{GeV}$ ). All the partial widths are given in $\mathrm{GeV}$

\begin{tabular}{|c|c|c|c|c|c|c|c|}
\hline \multirow{2}{*}{} & \multicolumn{3}{|c|}{$\mathrm{SM}$} & \multicolumn{3}{c|}{$\mathrm{EW} \nu_{R}$} & \\
\cline { 2 - 8 } & $\Gamma_{H \rightarrow g g}$ & $\Gamma$ & $\mathrm{BR}$ & $\Gamma_{\widetilde{H} \rightarrow g g}$ & $\Gamma$ & $\mathrm{BR}$ & \\
& $\propto \sigma_{g g \rightarrow H}$ & & & $\propto \sigma_{g g \rightarrow H}$ & & & \\
\hline$\widetilde{H} \rightarrow W^{+} W^{-}$ & $3.54 \mathrm{E}-04$ & $9.30 \mathrm{E}-04$ & $2.24 \mathrm{E}-01$ & $5.75 \mathrm{E}-04$ & $1.64 \mathrm{E}-04$ & $1.23 \mathrm{E}-01$ & 0.89 \\
$\widetilde{H} \rightarrow Z Z$ & $3.54 \mathrm{E}-04$ & $1.16 \mathrm{E}-04$ & $2.79 \mathrm{E}-02$ & $5.75 \mathrm{E}-04$ & $2.04 \mathrm{E}-05$ & $1.53 \mathrm{E}-02$ & 0.89 \\
$\widetilde{H} \rightarrow b \bar{H} \rightarrow \tau \bar{\tau}$ & $3.54 \mathrm{E}-04$ & $2.35 \mathrm{E}-03$ & $5.67 \mathrm{E}-01$ & $5.75 \mathrm{E}-04$ & $5.07 \mathrm{E}-04$ & $3.79 \mathrm{E}-01$ & 1.06 \\
$\widetilde{H} \rightarrow \gamma \gamma$ & $3.54 \mathrm{E}-04$ & $2.58 \mathrm{E}-04$ & $6.22 \mathrm{E}-02$ & $5.75 \mathrm{E}-04$ & $5.42 \mathrm{E}-05$ & $4.06 \mathrm{E}-02$ & 1.06 \\
\hline
\end{tabular}

\section{$\star$ Remarks}

- In all three examples when the SM-like singlet $H_{1}^{0}$ is the sub-dominant component of the $\widetilde{H}, H_{1 M}^{0}$ is the sub-dominant component as well. It should be the case because $H_{1 M}^{0}$ couples to the mirror fermions including six mirror quarks. All the mirror quarks in the $\mathrm{EW} \nu_{R}$ model are in the same mass order with top quark. They have significant contributions to the fermion loop in the gluon fusion which is the main production channel of the 125 GeV SM-like Higgs boson candidate. Therefore, the component of $H_{1 M}^{0}$ in the $\widetilde{H}$ should be small so that the ggF production cross section does not overly enhance. 
- As illustrated in Table 5.3, the ggF production cross section of $\widetilde{H}$ is almost twice as much as the ggF production of the SM Higgs boson. On the other hand, the branching ratios of $\widetilde{H} \rightarrow b \bar{b}, \tau \bar{\tau}, \gamma \gamma, W^{+} W^{-}, Z Z$ are about half of these of the SM Higgs boson in each channel. Eventually, the signal strengths of $\widetilde{H}$ decays in these channels are within the $1 \sigma$ constraints from CMS as seen in Fig. 5.2.

- The scanning procedure shows that Mr.Hyde scenario appears when the Yukawa couplings are smaller than in Dr.Jekyll scenario at the same mass of the mirror fermions. The signal strengths of $\widetilde{H}$ in the case of $M r$.Hyde satisfy the CMS constraints even when $\left|\lambda_{i} / 4 \pi\right|<1$. In this case, the $\widetilde{H}$ does not need to be in the strongly coupled regime.

\subsection{The Other Heavy States}

In this model, besides the two CP-even spin-zero states $\widetilde{H}^{\prime}, \widetilde{H}^{\prime \prime}$ from the mixing matrix in Eq. (5.3.22), we also have three CP-odd spin-zero states $H_{3}^{0}, H_{3 M}^{0}, H_{5}^{0}$. In this section, we discuss the predicted signal strengths of these spin-zero states except $H_{5}^{0}$, because it does not couple to both the SM and mirror fermions

\subsubsection{The heavy CP-even states}

For the heavy CP-even states, $\widetilde{H}^{\prime}, \widetilde{H}^{\prime \prime}$, the procedure of calculating their signal strengths is the same with $\widetilde{H}$ in the section 5.4. The only difference here is the appearance of the decay into the heavy fermions such as top quark and mirror quarks when these channels are kinematically allowed. Therefore, the total width 
of $\widetilde{H}^{\prime}, \widetilde{H}^{\prime \prime}$ is given by

$$
\begin{aligned}
\Gamma_{\widetilde{H}^{\prime}, \widetilde{H}^{\prime \prime}}= & \sum_{i=1}^{3} \Gamma_{\widetilde{H}^{\prime}, \widetilde{H}^{\prime \prime} \rightarrow q_{i}^{M} \bar{q}_{i}^{M}}+\sum_{j=1}^{3} \times \Gamma_{\widetilde{H}^{\prime}, \widetilde{H}^{\prime \prime} \rightarrow l_{j}^{M} \bar{l}_{j}^{M}} \\
& +\Gamma_{\widetilde{H}^{\prime}, \widetilde{H}^{\prime \prime} \rightarrow t \bar{t}}+\Gamma_{\widetilde{H}^{\prime}, \widetilde{H}^{\prime \prime} \rightarrow b \bar{b}} \\
& +\Gamma_{\widetilde{H}^{\prime}, \widetilde{H}^{\prime \prime} \rightarrow \tau \bar{\tau}}+\Gamma_{\widetilde{H}^{\prime}, \widetilde{H}^{\prime \prime} \rightarrow c \bar{c}}+\Gamma_{\widetilde{H}^{\prime}, \widetilde{H}^{\prime \prime} \rightarrow W+W^{-}} \\
& +\Gamma_{\widetilde{H}^{\prime}, \widetilde{H}^{\prime \prime} \rightarrow Z Z}+\Gamma_{\widetilde{H}^{\prime}, \widetilde{H}^{\prime \prime} \rightarrow g g}+\Gamma_{\widetilde{H}^{\prime}, \widetilde{H}^{\prime \prime} \rightarrow \gamma \gamma}
\end{aligned}
$$

In both five examples in Fig. 5.2, the next heavy state is $\widetilde{H}^{\prime}$. Here, we present the signal strength of $\widetilde{H}^{\prime} \rightarrow W^{+} W^{-}$of four examples in comparing with the data of CMS up to $600 \mathrm{GeV}$ in Fig. 5.3.

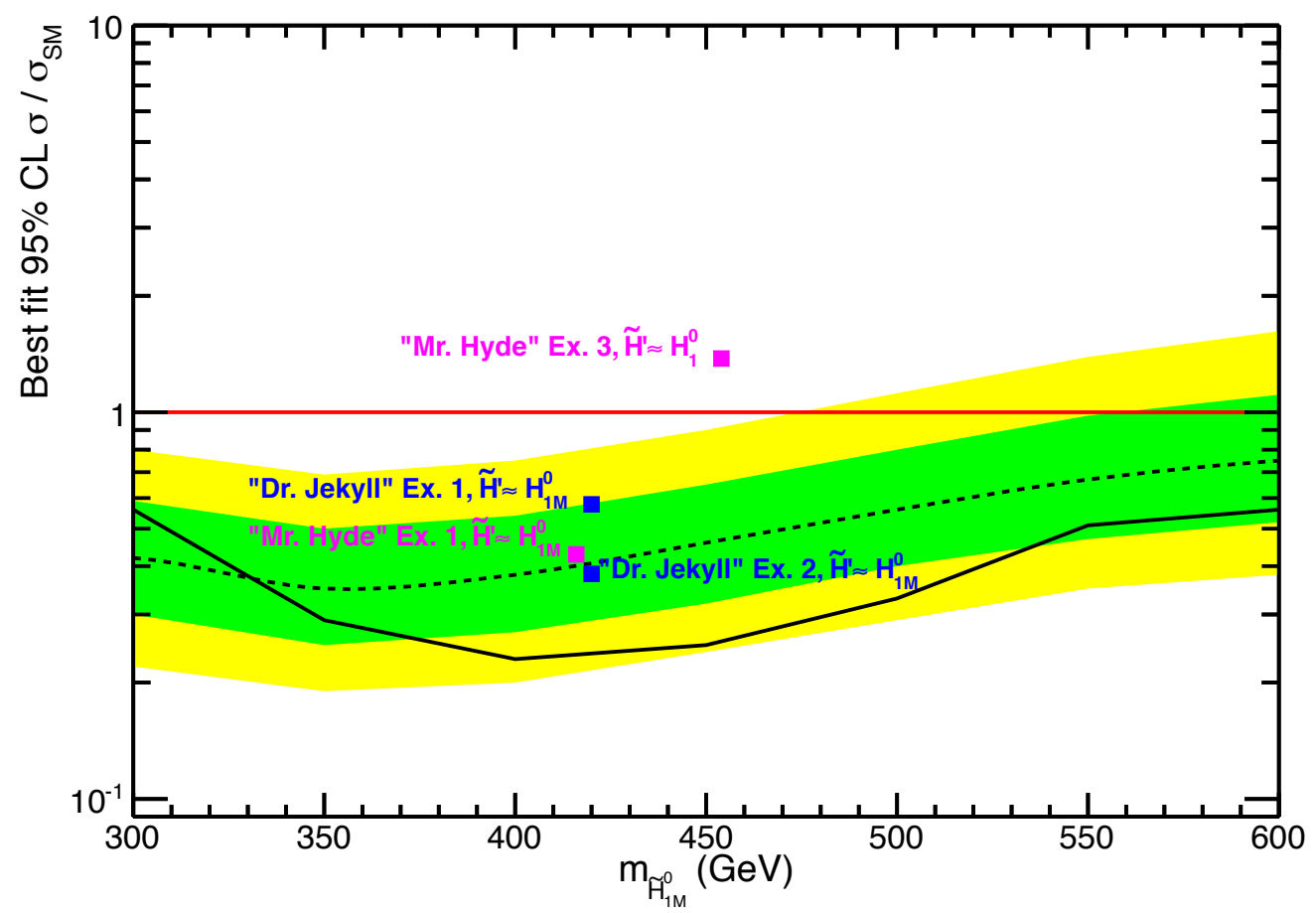

Figure 5.3. Predicted signal strength of $\widetilde{H}^{\prime} \rightarrow W^{+} W^{-}$in 4 example scenarios (blue and purple squares). The results of the search for SM-like Higgs boson up to $600 \mathrm{GeV}$ with the $1 \sigma$ (green band) and $2 \sigma$ (yellow band) limits on the $\mathrm{SM}$ background (dotted curve) and CMS data (solid black curve) are also displayed. 
In $M r . H y d e$ Ex. 2, $\widetilde{H}^{\prime}$ has the mass of $633 \mathrm{GeV}$ so that it is not included in Fig. 5.3. The mass here is just a particular example of $\widetilde{H}^{\prime}$. Among four examples, the signal strength of $\widetilde{H}^{\prime}$ in Dr.Jekyll Ex. 1, Ex. 2, and Mr.Hyde Ex. 1 is within the $1 \sigma \mathrm{SM}$ background. It means that the CMS data is not sensitive enough to confirm or rule out $\widetilde{H}^{\prime}$ in these examples.

One should note that the CMS data with the $1,2 \sigma$ bands here is with the Standard Model background. In the $\mathrm{EW} \nu_{R}$ model, the additional processes produced by the mirror fermions and the new scalars also contribute to the background. The background in the decay channel $\widetilde{H}^{\prime} \rightarrow W^{+} W^{-}$is expectedly larger than the SM background in the figure. Therefore, the signal strength of $\widetilde{H}^{\prime}$ in $M r$.Hyde Ex. 3 is most likely within the background if we include the background from the $\mathrm{EW} \nu_{R}$ model. It also means that Ex. 3 is not excluded by the data.

Moreover, in the high mass region, $\widetilde{H}^{\prime}$ is allowed to decay into the mirror fermions and new scalars of the EW $\nu_{R}$ model. The total width of $\widetilde{H}^{\prime}$ then can be large relatively to its mass. In this case, the signal of $\widetilde{H}^{\prime}$ does not appear as a narrow resonance above the background as the SM Higgs boson. ATLAS and CMS have constrained the upper limit on $\sigma \times B R(H \rightarrow \gamma \gamma)$ with the assumption in which the width of the Higgs boson $H$ is less than $10 \%$ of its mass $[35,36]$. Therefore, these upper limits do not apply to $\widetilde{H}^{\prime}$ absolutely.

\subsubsection{The CP-odd Heavy States}

In addition to three CP-even spin-zero states $\widetilde{H}, \widetilde{H}^{\prime}, \widetilde{H}^{\prime \prime}$, the $\mathrm{EW} \nu_{R}$ model also contains CP-odd spin-zero states - $H_{3}^{0}, H_{3 M}^{0}$. In this section, we show possibilities to probe the signal of the neutral pseudo-scalars in various decay channels at the LHC. To do so, we will investigate the product of production cross section and the branching ratio, a.k.a the absolute signal strength, in the $H_{3,3 M}^{0} \rightarrow \gamma \gamma, \tau \tau$ channels. We will also calculate the signal strengths $(\mu)$ for $H_{3}^{0}, H_{3 M}^{0}$ and the SM Higgs boson $H_{S M}$ in other channels. 


$$
\mu=\frac{\sigma\left(g g \rightarrow H_{3,3 M}^{0}\right) \operatorname{Br}\left(H_{3,3 M}^{0} \rightarrow X X\right)}{\sigma\left(g g \rightarrow H_{S M}\right) \operatorname{Br}\left(H_{S M} \rightarrow X X\right)}
$$

In this extension of the EW $\nu_{R}$ model, the degenerate masses of two $S U(2)_{D}$ custodial triplets are related by

$$
\frac{m_{H_{3}}^{2}}{m_{H_{3 M}}^{2}}=\frac{1}{1+c_{M}^{2}}
$$

We assume that the neutral states $H_{3}^{0}$ and $H_{3 M}^{0}$ obey this relationship, and use two cases of $s_{M}=0.168 ; 0.36$ for illustrative purpose.

\subsubsection{Ratio of Production Cross Sections}

At the LHC, $H_{3}^{0}, H_{3 M}^{0}$ are expected to be produced mainly through gluongluon fusion, similar to $H_{S M}$. By using an effective coupling approximation, we have

$$
R=\frac{\sigma\left(g g \rightarrow H_{3,3 M}^{0}\right)}{\sigma\left(g g \rightarrow H_{S M}\right)} \approx \frac{\Gamma\left(H_{3,3 M}^{0} \rightarrow g g\right)}{\Gamma\left(H_{S M} \rightarrow g g\right)} .
$$

$H_{3,3 M}^{0}$ do not couple directly to the gauge bosons $W, Z, \gamma$. And triplet couplings, such as $H_{3,3 M}^{0} H_{3,3 M}^{+} H_{3,3 M}^{-}, H_{3,3 M}^{0} H_{5}^{+} H_{5}^{-}, H_{3,3 M}^{0} H_{5}^{++} H_{5}^{--}$, are forbidden by CP conservation, see A.1. Therefore, only fermion loops involving the top quark and the mirror quarks contribute to the gluonic decay of $H_{3}^{0}$ and $H_{3 M}^{0}$ [38]:

$$
\begin{gathered}
\Gamma\left(H_{3,3 M}^{0} \rightarrow g g\right)=\frac{G_{F} \alpha_{s}^{2}}{16 \sqrt{2} \pi^{3}} m_{H_{3,3 M}^{0}}^{3}\left|\sum_{Q} g_{Q}^{H_{3,3 M}^{0}} F_{Q}^{H_{3,3 M}^{0}}\left(\tau_{f}\right)\right|^{2}, \\
F_{Q}^{H_{3,3 M}^{0}}\left(\tau_{f}\right)=\tau_{f} f\left(\tau_{f}\right), \\
\tau_{t}=4 m_{f}^{2} / m_{H_{3,3 M}^{0}}^{2},
\end{gathered}
$$

where $g_{Q}^{H_{3,3 M}^{0}}$ are the couplings of $H_{3}^{0}$ and $H_{3 M}^{0}$ to the top quark and mirror quarks, listed in Table 1. 
Here, $\sum_{Q}$ is summed over the top quark and the mirror quarks. However, the contributions from the mirror quarks can be suppressed due to the fact that the mirror-up quarks and the mirror-down quarks couple to $H_{3,3 M}^{0}$ with opposite signs. In this work, we particularly consider degenerate mirror fermion doublets, meaning $m_{u^{M}}=m_{d^{M}}$, for simplicity. As a result, the contributions from the mirror quarks cancel out. Thus only the top-quark loop appears in the production cross section of $H_{3}^{0}, H_{3 M}^{0}$. The ratios of production cross section are given by

$$
R_{H_{3}^{0}}=\tau_{t}^{2} \frac{\left|\tan \theta_{M} f\left(\tau_{t}\right)\right|^{2}}{\left|\tau_{t}+\left(\tau_{t}-1\right) f\left(\tau_{t}\right)\right|^{2}}
$$

for $H_{3}^{0}$, and

$$
R_{H_{3 M}^{0}}=\tau_{t}^{2} \frac{\left|s_{2 M} f\left(\tau_{t}\right)\right|^{2}}{\left|s_{2}\left(\tau_{t}+\left(\tau_{t}-1\right) f\left(\tau_{t}\right)\right)\right|^{2}}
$$

for $H_{3 M}^{0}$.

\subsubsection{The $\gamma \gamma$ Channel}

Similar to the gluonic decay, only fermion loops contribute to the partial width of $H_{3,3 M}^{0} \rightarrow \gamma \gamma$, given by [38]

$$
\Gamma\left(H_{3,3 M}^{0} \rightarrow \gamma \gamma\right)=\frac{g^{2} \alpha^{2} m_{H_{3,3 M}^{0}}^{3}}{256 \pi m_{W}^{2}}\left|\sum_{i} N_{c i} e_{i}^{2} g_{i} F_{i}^{H_{3,3 M}^{0}}\right|^{2} .
$$

Here, $i=$ top quark, six mirror quarks, and three charged mirror leptons. The total widths of $H_{3,3 M}^{0}$ are calculated by summing over all the partial widths.

$$
\begin{aligned}
\Gamma_{H_{3,3 M}}=\quad & \Gamma\left(H_{3,3 M}^{0} \rightarrow \gamma \gamma\right)+\Gamma\left(H_{3,3 M}^{0} \rightarrow g g\right) \\
& +\Gamma\left(H_{3,3 M}^{0} \rightarrow W^{+} W^{-}\right)+\Gamma\left(H_{3,3 M}^{0} \rightarrow Z Z\right) \\
& +\Gamma\left(H_{3,3 M}^{0} \rightarrow \tau \bar{\tau}\right)+\Gamma\left(H_{3,3 M}^{0} \rightarrow t \bar{t}\right) \\
& +\Gamma\left(H_{3,3 M}^{0} \rightarrow c \bar{c}\right)+\Gamma\left(H_{3,3 M}^{0} \rightarrow b \bar{b}\right) \\
& +\sum_{i=1}^{6} \Gamma\left(H_{3,3 M}^{0} \rightarrow q_{i}^{M} \bar{q}_{i}^{M}\right) \\
& +\sum_{j=1}^{3} \Gamma\left(H_{3,3 M}^{0} \rightarrow l_{j}^{M} \bar{l}_{j}^{M}\right)
\end{aligned}
$$


The branching ratio of $H_{3,3 M}^{0} \rightarrow \gamma \gamma$ is

$$
\operatorname{Br}\left(H_{3,3 M}^{0} \rightarrow \gamma \gamma\right)=\frac{\Gamma\left(H_{3,3 M}^{0} \rightarrow \gamma \gamma\right)}{\Gamma_{H_{3,3 M}^{0}}}
$$

The product of production cross section and the branching ratio in the decay channels $H_{3,3 M}^{0} \rightarrow \gamma \gamma$ is given by

$$
\sigma \times B R\left(H_{3,3 M}^{0} \rightarrow \gamma \gamma\right)=R \times \sigma\left(g g \rightarrow H_{S M}\right) \times B r\left(H_{3,3 M}^{0} \rightarrow \gamma \gamma\right)
$$

At any particular mass, $R_{H_{3,3 M}^{0}}$ and $B r\left(H_{3,3 M}^{0} \rightarrow \gamma \gamma\right)$ are calculated directly, while $\sigma\left(g g \rightarrow H_{S M}\right)$ is taken from the handbook of Higgs cross section at $8 \mathrm{TeV}$ [34]. To be consistent with the previous analysis, we also provide two scenarios which correspond to the dual nature of the $125-\mathrm{GeV}$ Higgs boson. We consider up- and down- members of mirror quark doublets to have degenerate masses. The first two generations of mirror quarks and all charged mirror leptons have the same mass, $m_{q_{1}^{M}}=m_{q_{2}^{M}}=m_{l^{M}}=102 \mathrm{GeV}$. The heaviest mirror quark generation has a mass $m_{q_{3}^{M}}=120 \mathrm{GeV}$. Masses of all right-handed neutrinos are at $M_{R}=50 \mathrm{GeV}$. Again, these numbers here are for illustrative purpose.

- In the case of Dr. Jekyll, as $\widetilde{H} \sim H_{1}^{0}$, the mixing angles are $s_{2}=0.92, s_{2 M}=$ $0.16, s_{M}=0.36$, which corresponds to the first example in the section 5.5.1. In Figure 5.4, we present the dependence of the production cross section times branching ratio of $H_{3,3 M}$ on mass. Moreover, the next heavy CP-even state is $\tilde{H}^{\prime}$ with the mass of $m_{\widetilde{H}^{\prime}}=420 \mathrm{GeV}$ in this case. So we incorporate the production cross section times branching ratio of $\widetilde{H}^{\prime} \rightarrow \gamma \gamma$ also. 


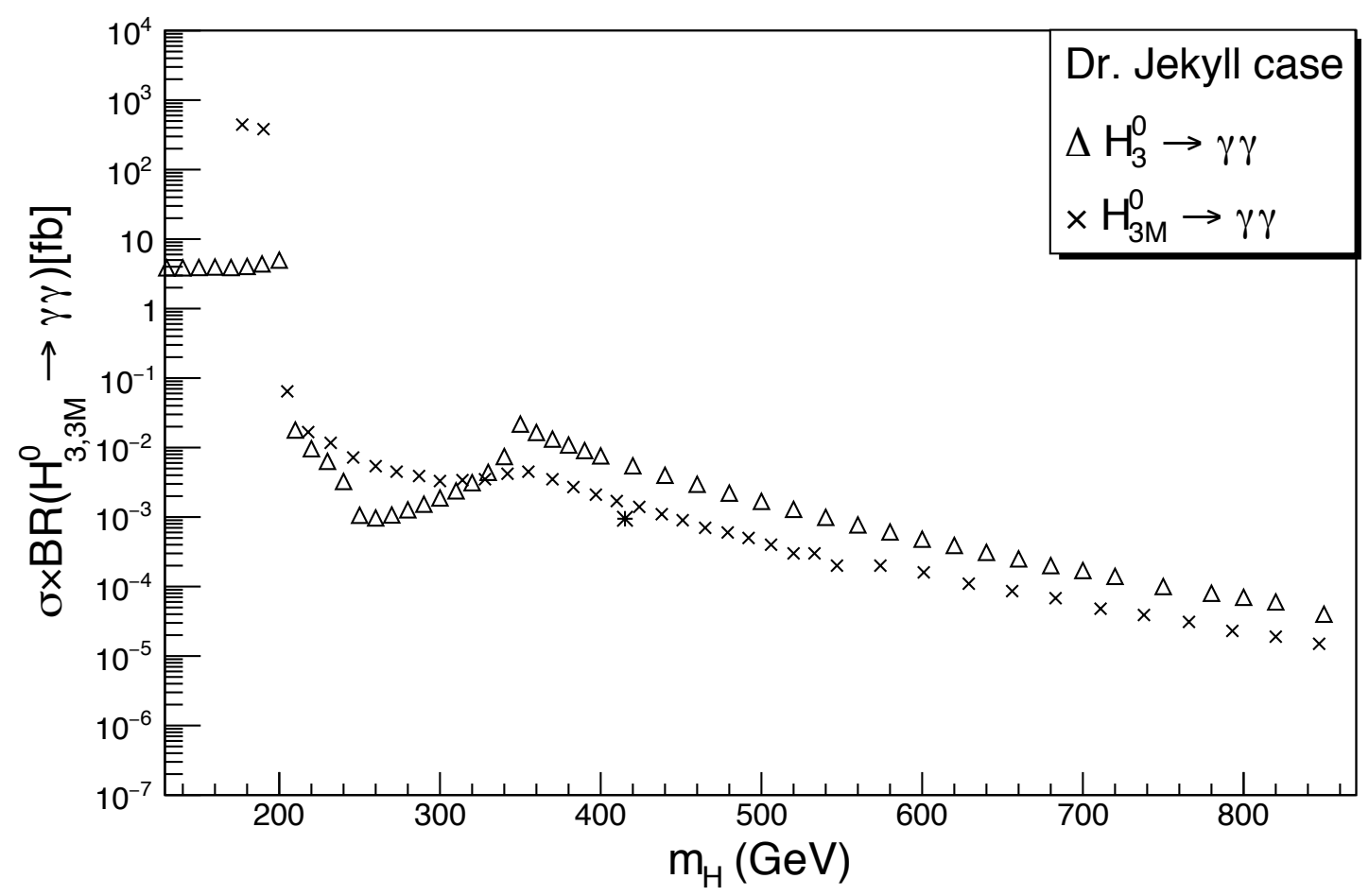

Figure 5.4. The production cross section times branching ratio in $\gamma \gamma$ channel of $H_{3}^{0}$ and $H_{3 M}^{0} \cdot m_{H_{3}^{0}}=130-850 \mathrm{GeV}, m_{H_{3 M}^{0}}=177-870 \mathrm{GeV}$

- In the other case when $H_{1}^{0}$ is sub-dominant in $\widetilde{H}$ or $M r$. Hyde, a set of parameters is chosen as $s_{2}=0.514, s_{2 M}=0.841, s_{M}=0.168$ corresponding to Example 2 in the section 5.5.2. In this scenario, all the heavy CP-even states are above $600 \mathrm{GeV}$. Therefore, we just present the dependence of the production cross section times branching ratio of $H_{3,3 M} \rightarrow \gamma \gamma$ in Figure 5.5. 


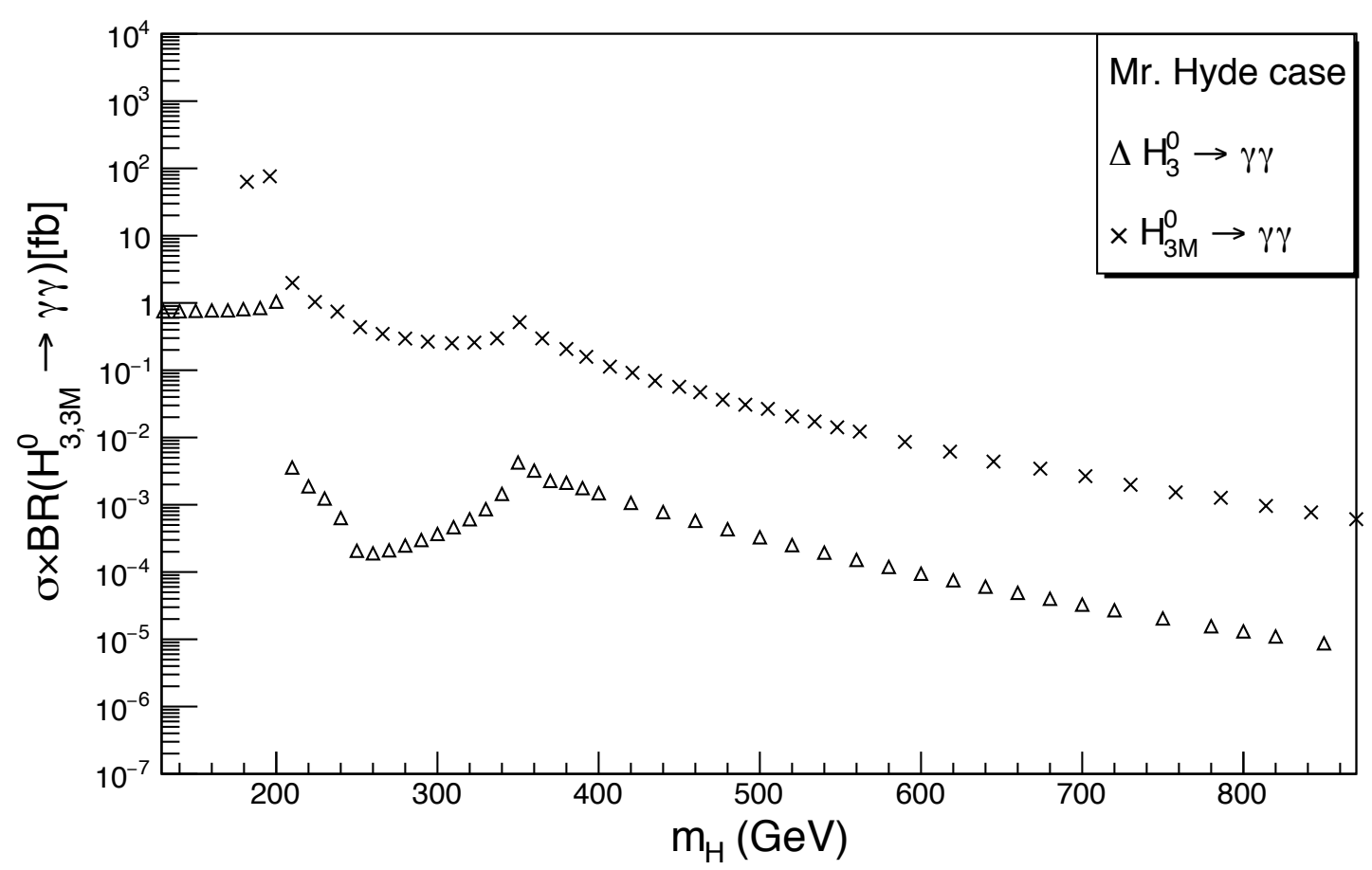

Figure 5.5. The production cross section times branching ratio in $\gamma \gamma$ channel of $H_{3}^{0}$ and $H_{3 M}^{0} \cdot m_{H_{3}^{0}}=130-750 \mathrm{GeV}, m_{H_{3 M}^{0}}=182-870 \mathrm{GeV}$

\section{$\star$ Remarks:}

- In Figs. 5.4, 5.5, the production cross section of $q+\bar{q} \rightarrow H_{3,3 M}^{0}$ is calculated based on the gluon fusion production of $H_{S M}$ at the same mass based on Eq. (5.6.13) at $8 \mathrm{TeV}$ LHC.

- Below fermionic thresholds, $2 m_{q_{1,2}^{M}}$ and $2 m_{l^{M}}$, the $\sigma \times B R$ of $H_{3,3 M}^{0}$ can be larger than what is measured by ATLAS and CMS for heavy SM-like scalar. To be conservative, we can exclude $m_{H_{3}^{0}} \lesssim 150 \mathrm{GeV}$ and $m_{H_{3 M}^{0}} \lesssim$ $205 \mathrm{GeV}$ (Fig. 5.4) and $\lesssim 210 \mathrm{GeV}$ (Fig. 5.5). However, in the other sets of parameters, the $\sigma \times B R$ could be well below the upper limit set by ATLAS and CMS. 
- As $m_{H_{3}^{0}}$ increases, more mirror fermionic decay channels are kinematically allowed. On the other hand, the production cross section decreases. The branching ratios of $H_{3,3 M}^{0} \rightarrow \gamma \gamma$ therefore decrease rapidly beyond the thresholds, $2 m_{q_{1,2}^{M}}, 2 m_{q_{3}^{M}}, 2 m_{l^{M}}, 2 m_{t}$. As a result, the $\sigma \times B R$ in both the in both cases are below the experimental upper limits.

- At the same mass, the $\sigma \times B R$ of CP-odd spin-zero states are generally larger than those of CP-even scalars. Consequently, it is easier to detect CP-odd spin-0 states than the CP-even ones.

\subsubsection{The $\tau \bar{\tau}$ Channel}

ATLAS [39] and CMS [40] also reported their new results in $\tau \bar{\tau}$ channel. Although, the main aim of their reports is to look for MSSM neutral boson, they provide a model-independent limit on the production cross section times branching ratio of a general spin-zero state. Therefore, in this part, we investigate the signal strength of the decay $H_{3,3 M}^{0} \rightarrow \tau \bar{\tau}$ with two sets of parameters considered in the previous section.

- In the Dr. Jekyll case. 


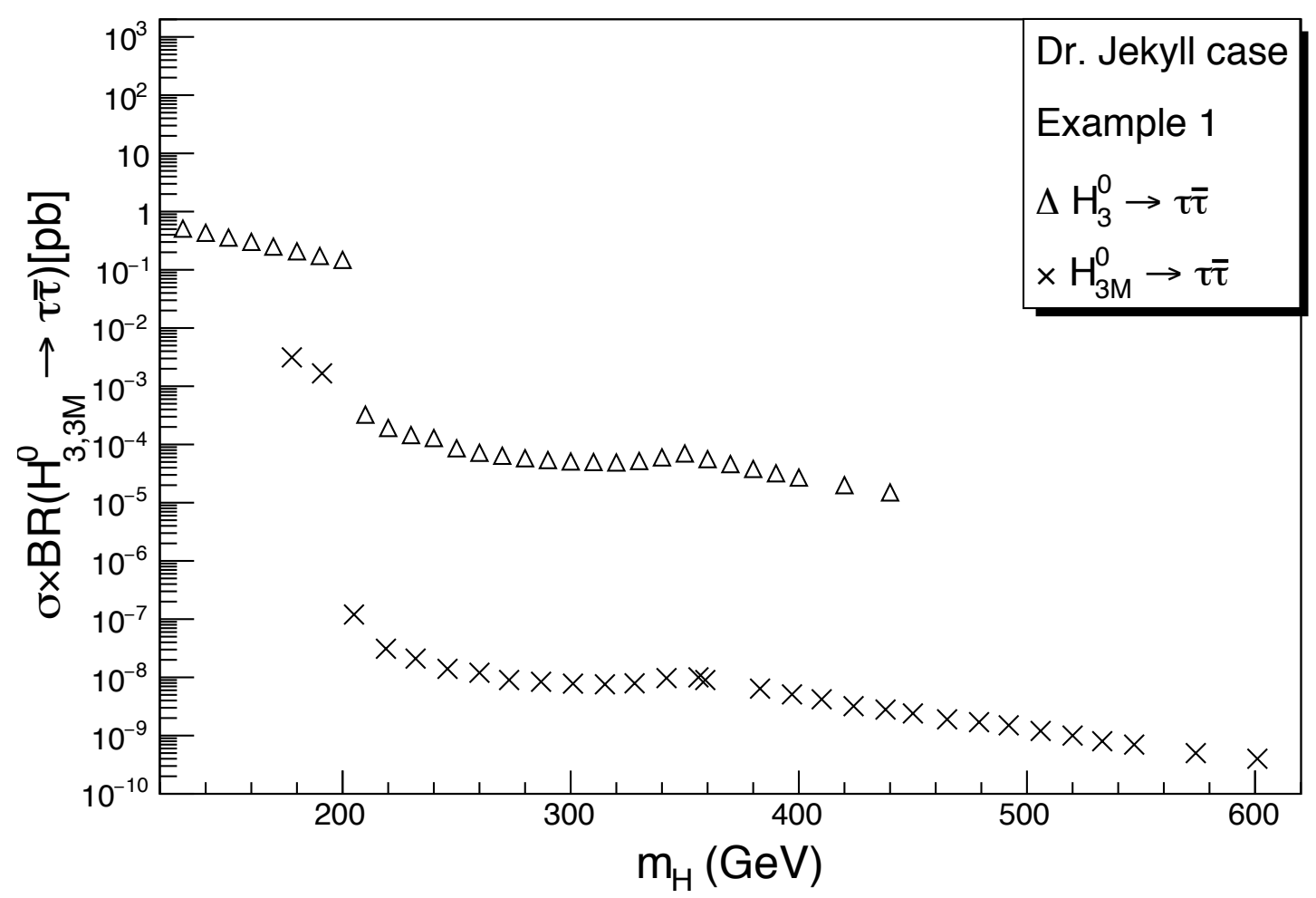

Figure 5.6. The production cross section times branching ratio in $\tau \bar{\tau}$ channel of $H_{3}^{0}$ and $H_{3 M}^{0} \cdot m_{H_{3}^{0}}=130-440 \mathrm{GeV}, m_{H_{3 M}^{0}}=177-601 \mathrm{GeV}$

- In the Mr.Hyde case. 


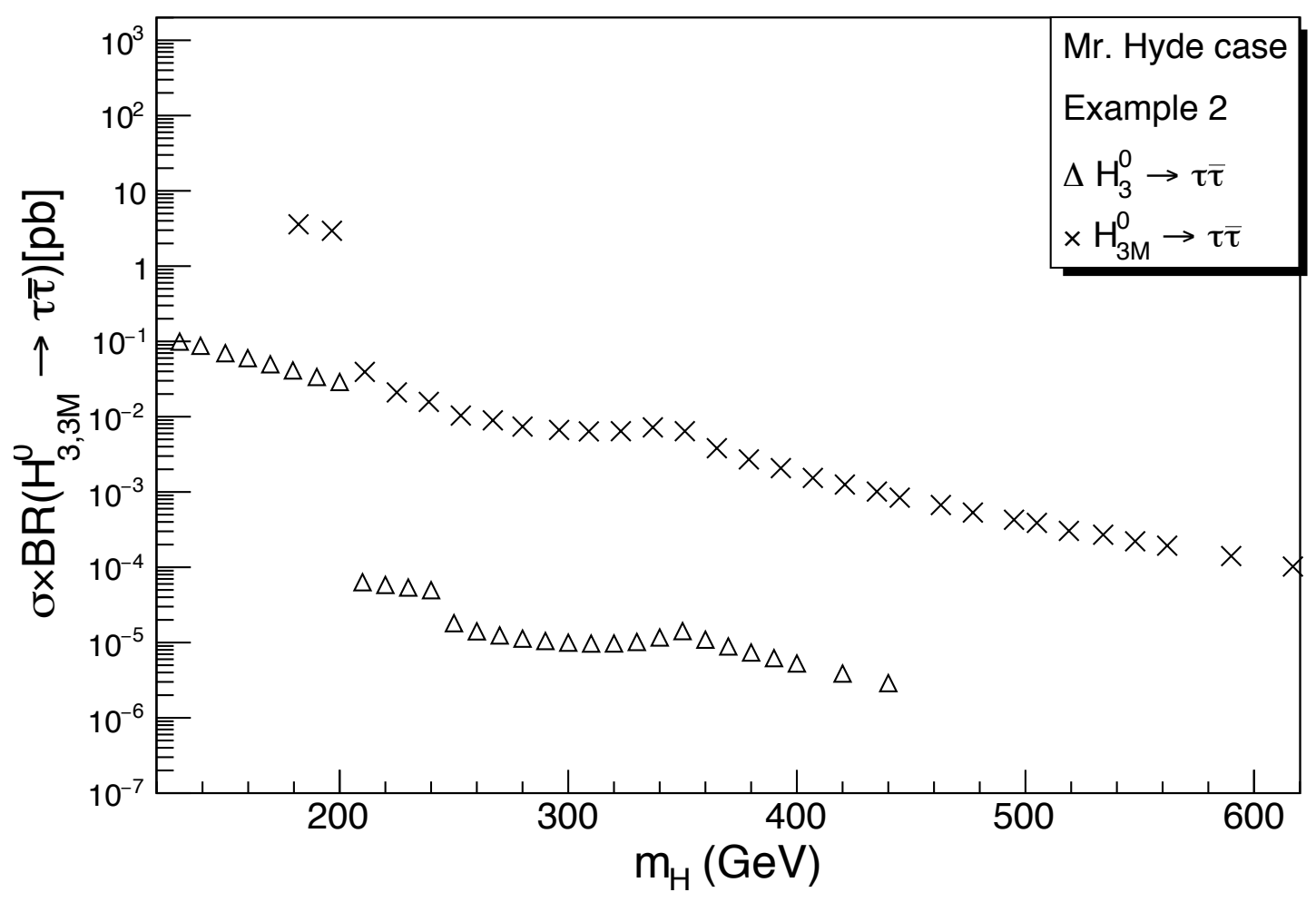

Figure 5.7. The production cross section times branching ratio in $\tau \bar{\tau}$ channel of $H_{3}^{0}$ and $H_{3 M}^{0} \cdot m_{H_{3}^{0}}=130-440 \mathrm{GeV}, m_{H_{3 M}^{0}}=182-618 \mathrm{GeV}$

\section{$\star$ Remarks:}

- In both cases, the $\sigma \times B R$ can exceed the upper limit from ATLAS and CMS before the thresholds of 2 times the mass of the lightest mirror fermions, which is $204 \mathrm{GeV}$. It happens because, unlike SM Higgs, the decay processes such as $H_{3,3 M}^{0} \rightarrow W W / Z Z$ occur only at the loop level, and their partial widths are relatively small. It means that the decay of $H_{3,3 M}^{0} \rightarrow \tau \bar{\tau}$ is sizable comparing to the other channels. Consequently, the branching ratios of $H_{3,3 M}^{0} \rightarrow \tau \bar{\tau}$ are not as small as in the Standard Model. Hence, the $\sigma \times B R$ for this channel is one order above the upper limits set by ATLAS and CMS. 
However, we already excluded this mass region of this particular parameter set based on the diphoton analysis in the last section.

- After passing the first threshold, the $\sigma \times B R$ of both $H_{3,3 M}^{0} \rightarrow \tau \bar{\tau}$ decrease rapidly, because the total widths $\Gamma_{H_{3,3 M}^{0}}$ are dominated by the fermionic decays. Then they reach another peak at $2 m_{t}$. Over the entire region after the first threshold, the $\sigma \times B R$ for both $H_{3,3 M}^{0}$ are below the limits.

\subsubsection{The $W W / Z Z$ Channels}

In this model, the pseudo-scalars $H_{3,3 M}^{0}$ do not couple directly to $W^{ \pm}$and $Z$. Decay processes $H_{3,3 M}^{0} \rightarrow W W / Z Z$ occur only at loop levels. One would expect these processes will be highly suppressed. To prove that, we calculate the signal strengths $(\mu)$ for $H_{3}^{0} \rightarrow W W / Z Z$ and $H_{S M} \rightarrow W W / Z Z$. $\mu$ is defined in Eq. (5.6.2).

$$
\begin{aligned}
\mu_{V V} & =\frac{\sigma\left(g g \rightarrow H_{3}^{0}\right) \operatorname{Br}\left(H_{3}^{0} \rightarrow V V\right)}{\sigma\left(g g \rightarrow H_{S M}\right) \operatorname{Br}\left(H_{S M} \rightarrow V V\right)} \\
& =R_{H_{3}^{0}} \frac{\operatorname{Br}\left(H_{3}^{0} \rightarrow V V\right)}{\operatorname{Br}\left(H_{S M} \rightarrow V V\right)},
\end{aligned}
$$

where $V=W, Z$. Once again, $\operatorname{Br}\left(H_{S M} \rightarrow V V\right)$ is taken from [34], while the ratio of production cross sections $R_{H_{3}^{0}}$ in Eq. (5.6.8) and $\mathrm{Br}\left(H_{3}^{0} \rightarrow V V\right)$ are calculated directly. At one loop order, the partial decay width for these processes are [41]

- $H_{3}^{0} \rightarrow W W$

$$
\begin{gathered}
\Gamma\left(H_{3}^{0} \rightarrow W W\right)=\frac{3^{2} g^{6}\left(m_{H_{3}^{0}}^{2}-4 m_{W}^{2}\right)^{3 / 2}}{2^{14} \pi^{5} m_{W}^{2}}\left|A_{W W}\right|^{2} \\
A_{W W}=m_{t}^{2} t_{M} A_{t}^{W}-m_{b}^{2} t_{M}^{2} A_{b}^{W}+\frac{m_{l^{M}}^{2}}{\sqrt{2}} A_{l^{M}}^{W}+\frac{M_{R}^{2}}{\sqrt{2} c_{M}} A_{\nu_{R}}^{W} ;
\end{gathered}
$$

- $H_{3}^{0} \rightarrow Z Z$

$$
\Gamma\left(H_{3}^{0} \rightarrow Z Z\right)=\frac{3^{2} g^{6}\left(m_{H_{3}^{0}}^{2}-4 m_{Z}^{2}\right)^{3 / 2}}{2^{15} \pi^{5} m_{W}^{2}}\left|A_{Z Z}\right|^{2}
$$




$$
A_{Z Z}=m_{t}^{2} t_{M} A_{t}^{Z}-m_{b}^{2} t_{M} A_{b}^{Z}+\frac{m_{l^{M}}^{2}}{\sqrt{2}} A_{l^{M}}^{Z}
$$

$A_{f}^{W / Z}$ are amplitudes with the top and the bottom quarks, the mirror charged leptons, and the right-handed neutrinos in the loops. They have specific forms given in Appendix A.3.

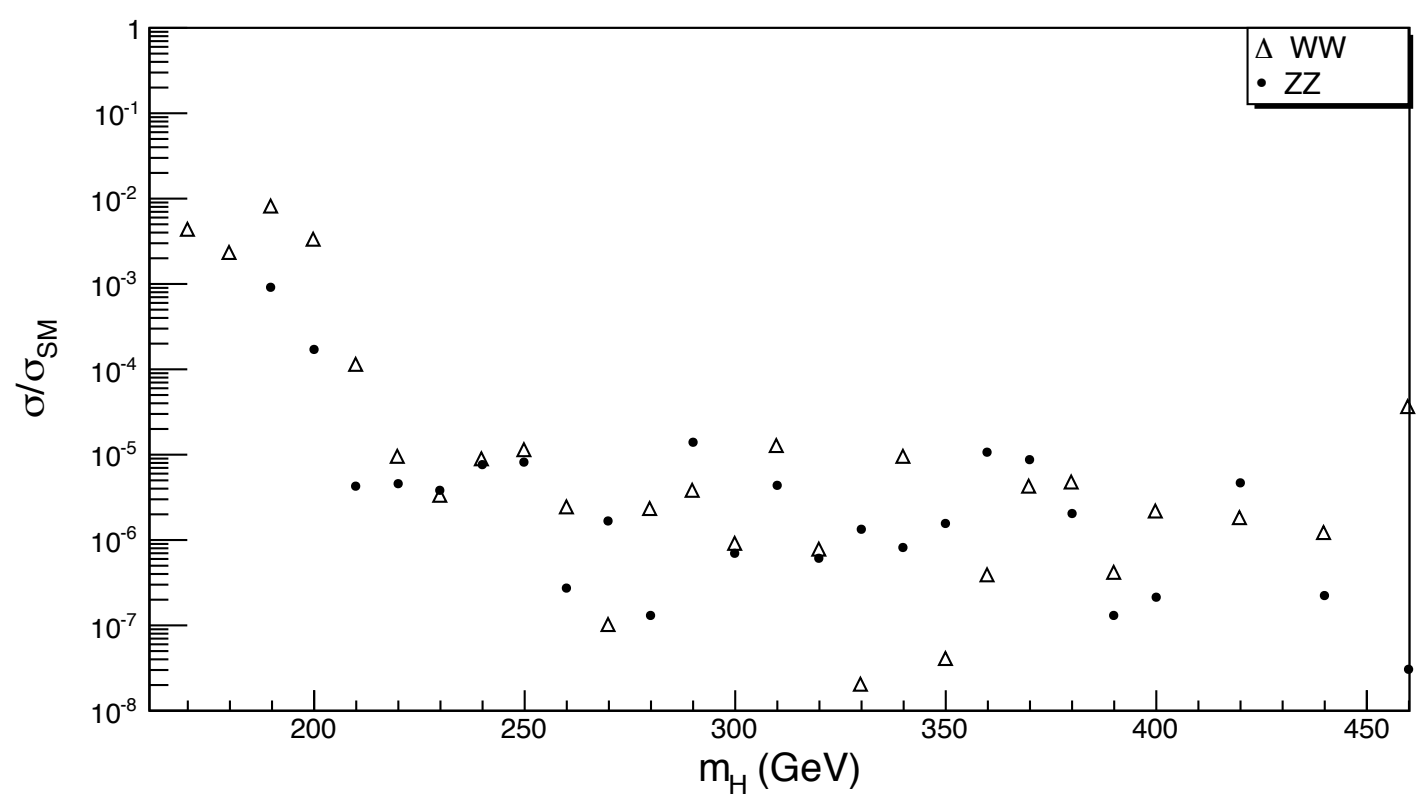

Figure 5.8. Ratio of strength signal in $W W / Z Z$ channel of $H_{3}^{0}$ comparing to $H_{S M}$

One can realize that all the contributions of the mirror quark are cancelled out in the Eqs. (5.6.16), (5.6.18) due to the opposite sign in the coupling of up- and down-mirror quarks. Also, we consider the case of the degenerate mass among mirror quarks in a family. As seen in Figure. 5.8, the signal strengths of $H_{3}^{0}$ in vector boson channels are highly suppressed in comparison to $H_{S M}$ in the $W W / Z Z$ decay channels. 


\subsection{Summary of The Chapter}

The discovery of the $125 \mathrm{GeV}$ SM-like Higgs boson is an important test of the $\mathrm{EW} \nu_{R}$ model together with the electroweak precision constraints. The $125 \mathrm{GeV}$ resonance is consistent with the Standard Model prediction in terms of the signal strength which is defined as the product of the production and branching ratio as in Eq. 5.4.19. Nevertheless, it is insufficient to conclude whether the newly discovered resonance is the definite SM Higgs boson or just an impostor because the lack of the explicit measurements of decay widths of the $125 \mathrm{GeV}$ resonance.

The $\mathrm{EW} \nu_{R}$ model was originally built to explain the nature of the neutrino mass by renovating the idea of mirror fermions and the Georgi-Machacek scalar potential. In the model, the right-handed neutrinos belong to $S U(2)_{W}$ doublets with their masses at the order of few hundred GeVs. The discovery of the 125 GeV SM-like Higgs boson led us to extend the minimal $\mathrm{EW} \nu_{R}$ model in the scalar sector by adding one more complex doublet. After considering the mixing between three $S U(2)$ singlets $H_{1}^{0}, H_{1 M}^{0}, H_{1}^{0 \prime}$ we have three mass eigenstates $\widetilde{H}, \widetilde{H}^{\prime}, \widetilde{H}^{\prime \prime}$ with the increasing order in their masses.

We scanned the parameter space to look for the candidate of the $125 \mathrm{GeV}$ SM-like Higgs boson under two condition: 1) The lightest mixing state, $\widetilde{H}$, has the mass in the region of $125 \mathrm{GeV} ; 2)$ The signal strength of $\widetilde{H}$ satisfies the $1 \sigma$ constraints from the experimental results of CMS. We found many combinations of the parameter space satisfying these two conditions. These combinations can be classified into two scenarios, Dr.Jekyll and Mr.Hyde, where the natures of the $125 \mathrm{GeV}$ Higgs boson candidate are very different but they still have the signal strengths to be consistent with the experimental results.

In the case of Dr.Jekyll, the $125 \mathrm{GeV}$ Higgs boson candidate is dominated by the SM-like component $H_{1}^{0}$. Naturally, the production cross section and the branching ratios of the Higgs boson candidate to are similar to the SM predic- 
tions. The signal strengths of the candidate satisfy the experimental constraints. On the other hand, the SM-like component is the sub-dominant in the case of Mr.Hyde so that the production cross section and the branching ratios are very different from the SM predictions. As we pointed out in three examples, the signal strengths of the candidate are still consistent with the experimental constraints if the component $H_{1 M}^{0}$ is also small in this case. This is the dual nature of the $125 \mathrm{GeV}$ SM-like Higgs boson candidate of the $\mathrm{EW} \nu_{R}$ model. We illustrate this special property of the model through 5 examples in Fig. 5.2.

We also investigated the signal strengths of the two heavier CP-even states $\widetilde{H}^{\prime}, \widetilde{H}^{\prime \prime}$ and two CP-odd spin-zero states $H_{3}^{0}, H_{3 M}^{0}$ in the main decay modes which have the upper limits from ATLAS/CMS measurements. So far, the existing experimental results can not confirm or rule out the existence of the CP-even states $\widetilde{H}^{\prime}, \widetilde{H}^{\prime \prime}$. While the two CP-odd scalars $H_{3}^{0}, H_{3 M}^{0}$ have a stronger signal generally except in the $W W / Z Z$ channels in which $H_{3}^{0}, H_{3 M}^{0} \rightarrow W W / Z Z$ at the loop level. Thus, we have several excluded regions of the parameter space based on the upper limit set by ATLAS/CMS. Again, the exclusion just applies for the particular parameter space in this thesis. 


\section{Chapter 6}

\section{Conclusions}

The Standard Model is the triumph and the tool for studying the physics of elementary particles. The discovery of the $125 \mathrm{GeV}$ SM-like Higgs boson is almost the last cornerstone for the unification of electromagnetic, weak and strong interactions [26]. Nearly all elementary phenomena are described by a single Lagrangian. Nevertheless, the non-zero mass of neutrinos indicates Physics beyond the Standard Model.

It is crucial to extend the Standard Model in order to generate the neutrino mass naturally. In neutrino model building, the seesaw mechanism is the most attractive idea. In this class of models, high scale sterile right-handed neutrinos are added to the fermion spectrum. However, the inert nature of the additional right-handed neutrinos makes this class of models to be impossible to test at current high energy colliders such as the LHC. One interesting alternative is the Left-Right symmetric extension of the Standard Model in which the SM is mapped to the right chirality in both fermion, scalar, and gauge boson sectors. Though the signal of the LR model can be probed at the LHC in principle, the newest search pushes the lower limit of the right-handed gauge boson $W_{R}$ to be more than 3.2 $\mathrm{TeV}$.

The EW $\nu_{R}$ model and its extension renovated the concept of mirror fermions 
proposed by Lee and Yang to incorporate the right-handed neutrinos into the SM doublets. By introducing the additional scalars singlets and triplets, one generates the small mass of the neutrino by seesawing between two energy scales: $\mathrm{keV}$ and $G e V$. The right-handed neutrinos in these models are non-sterile and have mass proportional to the electroweak scale. Thus, they are accessible at current and near future high energy colliders such as the LHC and ILC.

Having additional chiral doublets usually violates the electroweak precision data. However, the additional scalars in the $\mathrm{EW} \nu_{R}$ model, particularly the triplets, compensate for the contributions of the new fermions so that the model satisfies the electroweak precision constraints with a large parameter space. These constraints also indicate the mass splitting inside the fermion families and the scalar multiplets resulting in the signal of the new particles of the $\mathrm{EW} \nu_{R}$ model at hadron colliders.

In the extension of the $\mathrm{EW} \nu_{R}$ model, we incorporate the newly discovered 125 GeV SM-like Higgs boson successfully with a minimal extension in the scalar sector. More interestingly, the $125 \mathrm{GeV}$ Higgs boson can have two very different natures either SM-like or SM-unlike which both give the signal strengths consistent with the experimental measurements from the LHC. Besides, the study of the heavy scalars and the mirror fermions of the model would be very promising in the new phase of the LHC.

Originating from an ultimate goal to generate the tiny neutrino mass, the $\mathrm{EW} \nu_{R}$ model has been proved to pass all the important tests of a Beyond Standard Model: The electroweak precision tests and the $125 \mathrm{GeV}$ boson. The more investigation we did, the more self-consistence of the model we found. In the next few years, the LHC will further test the consistency of the EW sector of the SM and could reveal any new physics at the TeV scale in detail. This model of fertile right-handed neutrinos at electroweak scale would provide answers to known and unknown mysteries. 


\section{Appendices}

\section{A.1 The couplings in the Extended EW $\nu_{R}$ Model}

We present all the couplings of the scalars of the model.

Table 1. Yukawa couplings of the scalars with SM quarks and mirror-quarks in the $\mathrm{EW} \nu_{R}$ model.

\begin{tabular}{|c|c|c|c|}
\hline & SM Quarks & & Mirror Quarks \\
\hline$g_{H_{1}^{0} q \bar{q}}$ & $-\imath \frac{m_{q} g}{2 M_{W} s_{2}} \ldots(q=t, b)$ & $g_{H_{1 M}^{0} q^{M} \bar{q}^{M}}$ & $-\imath \frac{m_{q}^{M} g}{2 M_{W} s_{2 M}}$ \\
\hline$g_{H_{3}^{0} t \bar{t}}$ & $\imath \frac{m_{t} g s_{M}}{2 M_{W} c_{M}} \gamma_{5}$ & $g_{H_{3}^{0} u_{i}^{M} \bar{u}_{i}^{M}}$ & $-\imath \frac{m_{u_{i}^{M}} g s_{M}}{2 M_{W} c_{M}} \gamma_{5}$ \\
\hline$g_{H_{3 M}^{0} t \bar{t}}$ & $-\imath \frac{m_{t} g s_{2 M}}{2 M_{W} s_{2}} \gamma_{5}$ & $g_{H_{3 M}^{0} u_{i}^{M} \bar{u}_{i}^{M}}$ & $-\imath \frac{m_{u_{i}^{M}} g s_{2}}{2 M_{W} s_{2 M}} \gamma_{5}$ \\
\hline$g_{H_{3 M}^{0} b \bar{b}}$ & $\imath \frac{m_{b} g s_{2 M}}{2 M_{W} s_{2}} \gamma_{5}$ & $g_{H_{3 M}^{0} d_{i}^{M} \bar{d}_{i}^{M}}$ & $\imath \frac{m_{d_{i}^{M}} g s_{2}}{2 M_{W} s_{2 M}} \gamma_{5}$ \\
\hline
\end{tabular}


Table 2. Yukawa couplings of the scalars with SM quarks and mirror-quarks in the $\mathrm{EW} \nu_{R}$ model. (con't)

\begin{tabular}{|c|c|c|c|}
\hline$g_{H_{3 M}^{-} t \bar{b}}$ & $\begin{array}{c}\imath \frac{g s_{2 M}}{2 \sqrt{2} M_{W} s_{2} c_{M}}\left[m_{t}(1+\right. \\
\left.\left.\gamma_{5}\right)-m_{b}\left(1-\gamma_{5}\right)\right]\end{array}$ & $g_{H_{3 M}^{-} u_{i}^{M} \bar{d}_{i}^{M}}$ & $\begin{array}{c}\imath \frac{g s_{2}}{2 \sqrt{2} M_{W} s_{2 M} c_{M}}\left[m_{u_{i}^{M}}(1-\right. \\
\left.\left.\gamma_{5}\right)-m_{d_{i}^{M}}\left(1+\gamma_{5}\right)\right]\end{array}$ \\
\hline$g_{H_{1}^{0} l \bar{l}}$ & $\begin{array}{c}-\imath \frac{m_{l} g}{2 M_{W} s_{2}} \ldots .(l= \\
\tau, \mu, e)\end{array}$ & $g_{H_{1 M}^{0} l^{M} \bar{l}^{M}}$ & $-\imath \frac{m_{l}^{M} g}{2 M_{W} s_{2 M}}$ \\
\hline$g_{H_{3}^{0} l \bar{l}}$ & $-\imath \frac{m_{l} g s_{M}}{2 M_{W} c_{M}} \gamma_{5}$ & $g_{H_{3}^{0} l_{i}^{M} \bar{l}_{i}^{M}}$ & $\imath \frac{m_{l_{i}^{M}} g s_{M}}{2 M_{W} c_{M}} \gamma_{5}$ \\
\hline$g_{H_{3}^{-} \nu_{L} \bar{l}}$ & $-\imath \frac{g m_{l} s_{M}}{2 \sqrt{2} M_{W} c_{M}}\left(1-\gamma_{5}\right)$ & $g_{H_{3}^{-} \nu_{R i} \bar{l}_{i}^{M}}$ & $-\imath \frac{g m_{l_{i}^{M}} s_{M}}{2 \sqrt{2} M_{W} c_{M}}\left(1+\gamma_{5}\right)$ \\
\hline$g_{H_{3 M}^{0} l \bar{l}}$ & $\imath \frac{m_{l} g s_{2 M}}{2 M_{W} s_{2}} \gamma_{5}$ & $g_{H_{3 M}^{0} l_{i}^{M} \bar{l}_{i}^{M}}$ & $\imath \frac{m_{l_{i}^{M}} g s_{2}}{2 M_{W} s_{2 M}} \gamma_{5}$ \\
\hline$g_{H_{3 M}^{-} \nu_{L} \bar{\partial}}$ & $-\imath \frac{g m_{l} s_{2 M}}{2 \sqrt{2} M_{W} s_{2} c_{M}}\left(1-\gamma_{5}\right)$ & $g_{H_{3 M}^{-} \nu_{R i} \bar{l}_{i}^{M}}$ & $-\imath \frac{g m_{l_{i}^{M}} s_{2}}{2 \sqrt{2} M_{W} s_{2 M} c_{M}}\left(1+\gamma_{5}\right)$ \\
\hline
\end{tabular}


Table 3. Triple couplings of the scalars with one gauge boson. Here we use common factor: $\imath g\left(p-p^{\prime}\right)^{\mu}$, where $p\left(p^{\prime}\right)$ is the incoming momentum of the scalars.

\begin{tabular}{|c|c|c|c|}
\hline$g_{H_{5}^{0} H_{5}^{-} W^{+}}$ & $-\frac{\sqrt{3}}{2}$ & $g_{H_{5}^{++} H_{5}^{--} Z}$ & $-\frac{\left(1-2 s_{W}^{2}\right)}{c_{W}}$ \\
\hline$g_{H_{5}^{+} H_{5}^{--} W^{+}}$ & $-\frac{1}{\sqrt{2}}$ & $g_{H_{5}^{+} H_{5}^{-} Z}$ & $\frac{\left(1-2 s_{W}^{2}\right)}{2 c_{W}}$ \\
\hline$g_{H_{3}^{0} H_{3}^{-} W^{+}}$ & $-\frac{1}{2} s_{M}^{2}$ & $g_{H_{3}^{+} H_{3}^{-} Z}$ & $\frac{\left(1-2 s_{W}^{2}\right)}{2 c_{W}}$ \\
\hline$g_{H_{3 M}^{0} H_{3 M}^{-} W^{+}}$ & $\frac{1}{2}$ & $g_{H_{3 M}^{+} H_{3 M}^{-} Z}$ & $\frac{\left(1-2 s_{W}^{2}\right)}{2 c_{W}}$ \\
\hline$g_{H_{3}^{+} H_{5}^{--} W^{+}}$ & $-\frac{1}{\sqrt{2}} c_{M}$ & $g_{H_{3}^{+} H_{5}^{-} Z}$ & $-\frac{1}{2 c_{W}} c_{M}$ \\
\hline$g_{H_{3}^{0} H_{5}^{-} W^{+}}$ & $-\frac{1}{2} c_{M}$ & $g_{H_{3}^{0} H_{5}^{0} Z}$ & $\frac{1}{\sqrt{3}} \frac{c_{M}}{c_{W}}$ \\
\hline$g_{H_{5}^{0} H_{3}^{-} W^{+}}$ & $-\frac{1}{2 \sqrt{3}} c_{M}$ & $g_{G_{3}^{+} G_{3}^{-} Z}$ & $\frac{\left(1-2 s_{W}^{2}\right)}{2 c_{W}}$ \\
\hline$g_{G_{3}^{0} G_{3}^{-} W^{+}}$ & $-\frac{1}{2}$ & $g_{G_{3}^{0} H_{5}^{0} Z}$ & $\frac{1}{\sqrt{3}} \frac{s_{M}}{c_{W}}$ \\
\hline$g_{G_{3}^{+} H_{5}^{--} W^{+}}$ & $-\frac{1}{\sqrt{2}} s_{M}$ & $g_{G_{3}^{+} H_{5}^{-} Z}$ & $-\frac{1}{2 c_{W}} s_{M}$ \\
\hline$g_{G_{3}^{+} H_{5}^{--} W^{+}}$ & $-\frac{1}{\sqrt{2}} s_{M}$ & $g_{H_{1}^{0} G_{3}^{0} Z}$ & $\frac{s_{2}}{c_{W}}$ \\
\hline$g_{G_{3}^{0} H_{5}^{-} W^{+}}$ & $-\frac{1}{2} s_{M}$ & $g_{H_{1 M}^{0} G_{3}^{0} Z}$ & $\frac{s_{2 M}}{c_{W}}$ \\
\hline
\end{tabular}


Table 4. Triple couplings of the scalars with one gauge boson. Here we use common factor: $\imath g\left(p-p^{\prime}\right)^{\mu}$, where $p\left(p^{\prime}\right)$ is the incoming momentum of the scalars.

\begin{tabular}{|c|c|c|c|}
\hline$g_{H_{5}^{0} G_{3}^{-} W^{+}}$ & $\frac{1}{2 \sqrt{3}} s_{M}$ & $g_{H_{1}^{0 \prime} G_{3}^{0} Z}$ & $\sqrt{\frac{2}{3}} \frac{s_{M}}{c_{W}}$ \\
\hline$g_{H_{5}^{0} G_{3}^{-} W^{+}}$ & $\frac{1}{2 \sqrt{3}} s_{M}$ & $g_{H_{1}^{0} H_{3}^{0} Z}$ & $-\frac{s_{2} s_{M}}{2 c_{M} c_{W}}$ \\
\hline$g_{H_{1}^{0} G_{3}^{-} W^{+}}$ & $\frac{1}{2} s_{2}$ & $g_{H_{1 M}^{0} H_{3}^{0} Z}$ & $-\frac{s_{2 M} s_{M}}{2 c_{M} c_{W}}$ \\
\hline$g_{H_{1 M}^{0} G_{3}^{-} W^{+}}$ & $\frac{1}{2} s_{2 M}$ & $g_{H_{1}^{0 \prime} H_{3}^{0} Z}$ & $\sqrt{\frac{2}{3}} \frac{c_{M}}{c_{W}}$ \\
\hline$g_{H_{1}^{0 \prime} G_{3}^{-} W^{+}}$ & $\sqrt{\frac{2}{3}} s_{M}$ & $g_{H_{5}^{+} H_{5}^{-} \gamma}$ & $s_{W}$ \\
\hline$g_{H_{1}^{0} H_{3}^{-} W^{+}}$ & $-\frac{s_{2} s_{M}}{2 c_{M}}$ & $g_{H_{5}^{++} H_{5}^{--} \gamma}$ & $-2 s_{W}$ \\
\hline$g_{H_{1 M}^{0} H_{3}^{-} W^{+}}$ & $-\frac{s_{2 M} s_{M}}{2 c_{M}}$ & $g_{H_{3}^{+} H_{3}^{-} \gamma}$ & $s_{W}$ \\
\hline$g_{H_{1}^{0 \prime} H_{3}^{-} W^{+}}$ & $\sqrt{\frac{2}{3}} c_{M}$ & $g_{H_{3 M}^{+} H_{3 M}^{-} \gamma}$ & $s_{W}$ \\
\hline$g_{H_{1}^{0} H_{3 M}^{-} W^{+}}$ & $-\frac{s_{2 M}}{2 c_{M}}$ & $g_{G_{3}^{+} G_{3}^{-} \gamma}$ & $s_{W}$ \\
\hline$g_{H_{1 M}^{0} H_{3 M}^{-} W^{+}}$ & $\frac{s_{2}}{2 c_{M}}$ & $g_{H_{1}^{0} H_{3 M}^{0} Z}$ & $\frac{s_{2 M}}{2 c_{M}}$ \\
\hline & & $g_{H_{1 M}^{0} H_{3 M}^{0} Z}$ & $-\frac{s_{2}}{2 c_{M}}$ \\
\hline
\end{tabular}


Table 5. Triple couplings of the scalars with two gauge bosons. Here we use common factor: $\imath g M_{W} g^{\mu \nu}$.

\begin{tabular}{|c|c|c|c|}
\hline$g_{H_{5}^{0} W^{+} W^{-}}$ & $\frac{s_{M}}{\sqrt{3}}$ & $g_{H_{5}^{0} Z Z}$ & $-\frac{2}{\sqrt{3}} \frac{s_{M}}{c_{W}^{2}}$ \\
\hline$g_{H_{5}^{++} W^{-} W^{-}}$ & $\sqrt{2} s_{M}$ & $g_{H_{5}^{+} W^{-} Z}$ & $-\frac{s_{M}}{c_{W}}$ \\
\hline$g_{H_{1}^{0} W^{+} W^{-}}$ & $s_{2}$ & $g_{H_{1}^{0} Z Z}$ & $\frac{s_{2}}{c_{W}^{2}}$ \\
\hline$g_{H_{1 M}^{0} W^{+} W^{-}}$ & $s_{2 M}$ & $g_{H_{1 M}^{0} Z Z}$ & $\frac{s_{2 M}}{c_{W}^{2}}$ \\
\hline$g_{H_{1}^{0 \prime} W^{+} W^{-}}$ & $\frac{2 \sqrt{2}}{\sqrt{3}} s_{M}$ & $g_{H_{1}^{0 \prime} Z Z}$ & $\frac{2 \sqrt{2}}{\sqrt{3}} \frac{s_{M}}{c_{W}^{2}}$ \\
\hline
\end{tabular}


Table 6. Quartic couplings of the scalars with the gauge bosons. Common factor: $\imath g^{2} g^{\mu \nu}$.

\begin{tabular}{|l|c|c|c|}
\hline$g_{H_{5}^{0} H_{5}^{0} W^{+} W^{-}}$ & $\frac{5}{3}$ & $g_{H_{5}^{0} H_{5}^{0} Z Z}$ & $\frac{2}{3 c_{W}^{2}}$ \\
\hline$g_{H_{5}^{+} H_{5}^{-} W^{+} W^{-}}$ & $-\frac{3}{2}$ & $g_{H_{5}^{+} H_{5}^{-} Z Z}$ & $-\frac{\left(c_{W}^{4}+s_{W}^{4}\right)}{c_{W}^{2}}$ \\
\hline$g_{H_{5}^{++} H_{5}^{--} W^{+} W}-$ & 1 & $g_{H_{5}^{++} H_{5}^{--} Z Z}$ & $2 \frac{\left(1-2 s_{W}^{2}\right)^{2}}{c_{W}^{2}}$ \\
\hline$g_{H_{3}^{0} H_{3}^{0} W^{+} W^{-}}$ & $-\frac{\left(1+c_{M}^{2}\right)}{2}$ & $g_{H_{3}^{0} H_{3}^{0} Z Z}$ & $-\frac{1}{2 c_{W}^{2}}\left(1+3 c_{M}^{2}\right)$ \\
\hline$g_{H_{3}^{+} H_{3}^{-} W^{+} W^{-}}$ & $-\left(\frac{1}{2}+c_{M}^{2}\right)$ & $g_{H_{3}^{+} H_{3}^{-} Z Z}$ & $-\left[\frac{s_{M}^{2}}{2} \frac{\left(1-s_{W}^{2}\right)^{2}}{c_{W}^{2}}+c_{M}^{2} \frac{\left(c_{W}^{4}+s_{W}^{4}\right)}{c_{W}^{2}}\right.$ \\
\hline$g_{H_{3 M}^{0} H_{3 M^{0} W^{+} W}}$ & $-\frac{1}{2}$ & $g_{H_{3 M}^{0} H_{3 M}^{0} Z Z}$ & $\frac{1}{2 c_{W}^{2}}$ \\
\hline$g_{H_{3 M}^{+} H_{3 M}^{-} W^{+} W}$ & $-\frac{1}{2}$ & $g_{H_{3 M}^{+} H_{3 M}^{-} Z Z}$ & $-\frac{\left(1-2 s_{W}^{2}\right)^{2}}{2 c_{W}^{2}}$ \\
\hline$g_{G_{3}^{0} G_{3}^{0} W^{+} W^{-}}$ & $-\frac{\left(1+s_{M}^{2}\right)}{2}$ & $g_{G_{3}^{0} G_{3}^{0} Z Z}$ & $-\frac{1}{2 c_{W}^{2}}\left(1+3 s_{M}^{2}\right)$ \\
\hline$g_{G_{3}^{+} G_{3}^{-} W^{+} W^{-}}$ & $-\left(\frac{1}{2}+s_{M}^{2}\right)$ & $g_{G_{3}^{+} G_{3}^{-} Z Z}$ & $-\left[\frac{c_{M}^{2}}{2} \frac{\left(1-s_{W}^{2}\right)^{2}}{c_{W}^{2}}+s_{M}^{2} \frac{\left(c_{W}^{4}+s_{W}^{4}\right)}{c_{W}^{2}}\right.$ \\
\hline$g_{H_{1}^{0} H_{1}^{0} W^{+} W^{-}}$ & $\frac{1}{2}$ & $g_{H_{1}^{0} H_{1}^{0} Z Z}$ & $g_{H_{1 M}^{0} H_{1 M}^{0} Z Z}$ \\
\hline$g_{H_{1 M}^{0} H_{1 M}^{0} W^{+} W}$ & $\frac{1}{2}$ & $g_{H_{1}^{0 \prime} H_{1}^{0 \prime} Z Z}$ & $\frac{1}{2 c_{W}^{2}}$ \\
\hline$g_{H_{1}^{0 \prime} H_{1}^{0 \prime} W^{+} W^{-}}$ & $\frac{4}{3}$ & $\frac{1}{2 c_{W}^{2}}$ \\
\hline
\end{tabular}


Table 7. Quartic couplings of the scalars and gauge bosons. Common factor: $\imath g^{2} g^{\mu \nu}$.

\begin{tabular}{|c|c|c|c|}
\hline$g_{H_{5}^{+} H_{5}^{-} \gamma \gamma}$ & $-2 s_{W}^{2}$ & $g_{H_{5}^{+} H_{5}^{-} Z \gamma}$ & $-\frac{s_{W}}{c_{W}}\left(1-2 s_{W}^{2}\right)$ \\
\hline$g_{H_{5}^{++} H_{5}^{--} \gamma \gamma}$ & $8 s_{W}^{2}$ & $g_{H_{5}^{++} H_{5}^{--} Z \gamma}$ & $4 \frac{s_{W}}{c_{W}}\left(1-2 s_{W}^{2}\right)$ \\
\hline$g_{H_{3}^{+} H_{3}^{-} \gamma \gamma}$ & $-2 s_{W}^{2}$ & $g_{H_{3}^{+} H_{3}^{-} Z \gamma}$ & $-\frac{s_{W}}{c_{W}}\left(1-2 s_{W}^{2}\right)$ \\
\hline$g_{H_{3 M}^{+} H_{3 M}^{-} \gamma \gamma}$ & $-2 s_{W}^{2}$ & $g_{H_{3 M}^{+} H_{3 M}^{-} Z \gamma}$ & $-\frac{s_{W}}{c_{W}}\left(1-2 s_{W}^{2}\right)$ \\
\hline$g_{G_{3}^{+} G_{3}^{-} \gamma \gamma}$ & $-2 s_{W}^{2}$ & $g_{G_{3}^{+} G_{3}^{-} Z \gamma}$ & $-\frac{s_{W}}{c_{W}}\left(1-2 s_{W}^{2}\right)$ \\
\hline$g_{H_{1}^{0 \prime} H_{5}^{0} W^{+} W^{-}}$ & $\frac{\sqrt{2}}{3}$ & $g_{H_{1}^{0 \prime} H_{5}^{0} Z Z}$ & $-\frac{2 \sqrt{2}}{3 c_{W}^{2}}$ \\
\hline$g_{H_{3}^{+} H_{5}^{-} W^{+} W^{-}}$ & $-\frac{c_{M}}{2}$ & $g_{H_{3}^{+} H_{5}^{-} Z Z}$ & $c_{M} \frac{\left(1-2 s_{W}^{2}\right)}{c_{W}^{2}}$ \\
\hline$g_{H_{3}^{0} G_{3}^{0} W^{+} W^{-}}$ & $-\frac{c_{M} s_{M}}{2}$ & $g_{H_{3}^{0} G_{3}^{0} Z Z}$ & $-\frac{3}{2} \frac{c_{M} s_{M}}{c_{W}^{2}}$ \\
\hline$g_{H_{3}^{+} G_{3}^{-} W^{+} W^{-}}$ & $-c_{M} s_{M}$ & $g_{H_{3}^{+} G_{3}^{-} Z Z}$ & $-\frac{c_{M} s_{M}}{2 c_{W}^{2}}$ \\
\hline$g_{H_{5}^{+} G_{3}^{-} W^{+} W^{-}}$ & $-\frac{s_{M}}{2}$ & $g_{H_{5}^{+} G_{3}^{-} Z Z}$ & $\frac{\left(1-2 s_{W}^{2}\right)}{c_{W}^{2}}$ \\
\hline & & $g_{H_{3}^{+} H_{5}^{-} Z \gamma}$ & $c_{W} \frac{s_{W}}{c_{W}}$ \\
\hline & & & $s_{M}$ \\
\hline
\end{tabular}




\section{A.2 Loop Functions}

To express the contributions of scalars and fermions into the oblique parameters, $\widetilde{S}, \widetilde{T}$, at one loop level, it is convenient to use so-called one-point and twopoint functions [43]. Basically, they are the coefficients in the zeroth order of the expansion of the self-energy diagrams of the gauge vector bosons $\Pi_{\mu \nu}$. In general, these self-energy diagrams can be expressed as

$$
\Pi_{\mu \nu}=\Pi_{0} g_{\mu \nu}+\Pi_{2} q_{\mu} q_{\nu}
$$

For the purpose of calculating the oblique parameters, we can ignore the second order of the expansion. So in this thesis, $\Pi_{\mu \nu}=\Pi_{0} g_{\mu \nu}$. The loop diagrams involving one or two scalars or fermions appear in the form of the t'Hooft-Veltman integrals. They are expressed as [43] One-point integral:

$$
\int \frac{d^{4} k}{(2 \pi)^{4}} \frac{1}{\left(k^{2}-m^{2}\right)} \equiv \frac{i}{16 \pi^{2}} A_{0}\left(m^{2}\right)
$$

Two-point integrals:

$$
\begin{aligned}
& \int \frac{d^{4} k}{(2 \pi)^{4}} \frac{1}{\left(k^{2}-m_{1}^{2}\right)\left((k+q)^{2}-m_{2}^{2}\right)} \\
& \equiv \frac{i}{16 \pi^{2}} B_{0}\left(q^{2} ; m_{1}^{2}, m_{2}^{2}\right), \\
& \int \frac{d^{4} k}{(2 \pi)^{4}} \frac{k_{\mu} k_{\nu}}{\left(k^{2}-m_{1}^{2}\right)\left((k+q)^{2}-m_{2}^{2}\right)} \\
& \equiv \frac{i}{16 \pi^{2}} g_{\mu \nu} B_{22}\left(q^{2} ; m_{1}^{2}, m_{2}^{2}\right)
\end{aligned}
$$


In the dimensional regularization, these integrals can be simplified to

$$
\begin{aligned}
A_{0}\left(m^{2}\right) & =m^{2}\left(\Delta+1-\ln \left(m^{2}\right)\right) \\
B_{0}\left(q^{2} ; m_{1}^{2}, m_{2}^{2}\right) & =\Delta-\int_{0}^{1} d \mathrm{x} \ln (X-i \epsilon) \\
B_{22}\left(q^{2} ; m_{1}^{2}, m_{2}^{2}\right) & =\frac{1}{4}(\Delta+1)\left(m_{1}^{2}+m_{2}^{2}-\frac{q^{2}}{3}\right) \\
& -\frac{1}{2} \int_{0}^{1} d \mathrm{x} X \ln (X-i \epsilon)
\end{aligned}
$$

where

$$
\begin{aligned}
X & \equiv m_{1}^{2} \mathrm{x}+m_{2}^{2}(1-\mathrm{x})-q^{2} \mathrm{x}(1-\mathrm{x}) \\
\Delta & \equiv \frac{2}{4-d}+\ln (4 \pi)-\gamma
\end{aligned}
$$

in $d$ space-time dimensions with $\gamma=0.577216 \ldots$, the Euler's constant [45]. The integrals in eqns. (A.2.6), (A.2.7) can be calculated numerically up to desired accuracy. Note that these equations involve the logarithm of a dimensionful quantity, $X$ and the scale of this logarithm is hidden in the $2 /(4-d)$ term in $\Delta$ (refer to section 7.5 of [45]). We also have [46]

$$
\begin{aligned}
B_{0}\left(0 ; m_{1}^{2}, m_{2}^{2}\right) & =\frac{A_{0}\left(m_{1}^{2}\right)-A_{0}\left(m_{2}^{2}\right)}{m_{1}^{2}-m_{2}^{2}}, \\
4 B_{22}\left(0 ; m_{1}^{2}, m_{2}^{2}\right) & =\mathcal{F}\left(m_{1}^{2}, m_{2}^{2}\right)+A_{0}\left(m_{1}^{2}\right)+A_{0}\left(m_{2}^{2}\right),
\end{aligned}
$$

where

$$
\begin{aligned}
\mathcal{F}\left(m_{1}^{2}, m_{2}^{2}\right)= & \frac{m 1^{2}+m_{2}^{2}}{2}-\frac{m_{1}^{2} m_{2}^{2}}{m_{1}^{2}-m_{2}^{2}} \ln \left(\frac{m_{1}^{2}}{m_{2}^{2}}\right), \\
& \text { if } m_{1} \neq m_{2}, \\
= & 0 \quad \text { if } m_{1}=m_{2} .
\end{aligned}
$$

Note that

$$
\mathcal{F}\left(m_{1}^{2}, m_{2}^{2}\right)=\mathcal{F}\left(m_{2}^{2}, m_{1}^{2}\right)
$$


So then

$$
\begin{aligned}
B_{22}\left(q^{2} ; m_{1}^{2}, m_{2}^{2}\right) & =B_{22}\left(q^{2} ; m_{2}^{2}, m_{1}^{2}\right) \\
B_{0}\left(q^{2} ; m_{1}^{2}, m_{2}^{2}\right) & =B_{0}\left(q^{2} ; m_{2}^{2}, m_{1}^{2}\right) .
\end{aligned}
$$

While evaluating the fermion loops which contribute to the oblique parameters following two-point loop integrals are useful (refer section 21.3 of [45]):

$$
\begin{aligned}
& B_{1}\left(q^{2} ; m_{1}^{2}, m_{2}^{2}\right)=\int_{0}^{1} d \mathrm{x}(1-\mathrm{x}) \ln \left(\frac{X-i \epsilon}{M^{2}}\right), \\
& B_{2}\left(q^{2} ; m_{1}^{2}, m_{2}^{2}\right)=\int_{0}^{1} d \mathrm{xx}(1-\mathrm{x}) \ln \left(\frac{X-i \epsilon}{M^{2}}\right),
\end{aligned}
$$

where $X$ is as defined in eqn. (A.2.8). The logarithms in these integrals involve a mass scale $M$. All the terms, which depend on this scale cancel while evaluating the final expressions for oblique parameters. For $m_{1}=m_{2}=m$ and $q^{2}=M_{Z}^{2}$,

$$
\begin{aligned}
B_{1}\left(M_{Z}^{2} ; m^{2}, m^{2}\right) & =-1-\frac{G(\mathrm{x})}{4}+\ln \left(\frac{m^{2}}{M^{2}}\right), \\
B_{2}\left(M_{Z}^{2} ; m^{2}, m^{2}\right) & =\frac{1}{18}\left[-\frac{3}{2} G(x)(2 x+1)\right. \\
& \left.+\left(-12 x-5+3 \ln \left(\frac{m^{2}}{M^{2}}\right)\right)\right],
\end{aligned}
$$

where

$$
G(x)=-4 \sqrt{4 x-1} \operatorname{Arctan}\left(\frac{1}{\sqrt{4 x-1}}\right)
$$

While deriving $\widetilde{T}_{\text {fermion }}$ in eqn.(4.1.9) we need to evaluate integrals in eqn. (A.2.15) for $q=0$ and $m_{1} \neq m_{2}$. One of the integrals, which appear in this calculation is

$$
\begin{aligned}
& \int_{0}^{1} d x\left(m_{1}^{2} x+m_{2}^{2}(1-x)\right) \ln \left(\frac{m_{1}^{2} x+m_{2}^{2}(1-x)}{M^{2}}\right) \\
& =\frac{\left(m_{2}^{4}-m_{1}^{4}\right)+2 m_{1}^{4} \ln \left(\frac{m_{1}^{2}}{M^{2}}\right)-2 m_{2}^{4} \ln \left(\frac{m_{1}^{2}}{M^{2}}\right)}{4\left(m_{1}^{2}-m_{2}^{2}\right)} .
\end{aligned}
$$


Using the loop integrals and functions defined and enlisted in this appendix we can derive the expressions for the oblique parameters, which are suitable for the numerical analysis

\section{A.3 Amplitude of $H_{3}^{0} \rightarrow W W / Z Z$}

The processes such as $H_{3}^{0} \rightarrow W W / Z Z$ in this model only take place at loop level. At 1 loop, the Feynman diagrams are:

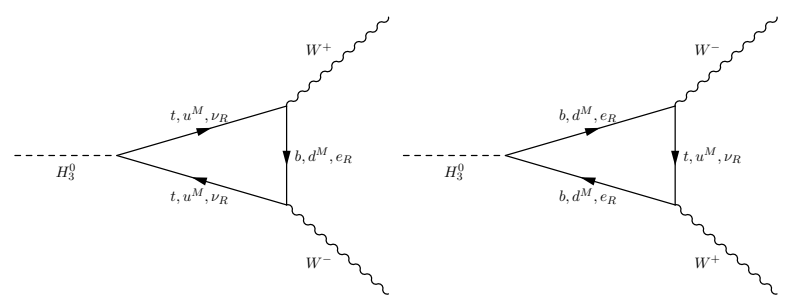

Figure 1. Feynman diagram of $H_{3}^{0} \rightarrow W^{+} W^{-}$. We have three generations of mirror quarks and three generations of mirror leptons

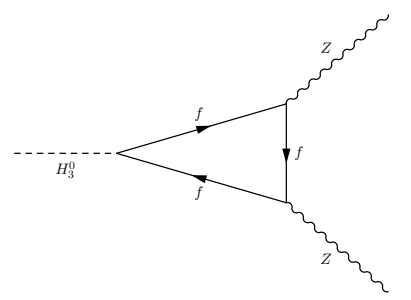

Figure 2. Feynman diagram of $H_{3}^{0} \rightarrow Z Z$. Here, $f=u_{1}^{M}, d_{1}^{M}, u_{2}^{M}, d_{2}^{M}, u_{3}^{M}, d_{3}^{M}$, and three charged mirror leptons $l^{M}$

With the couplings in Table 1, the amplitude can be expressed as [41]

$$
A\left(H_{3}^{0} \rightarrow V V\right)=m_{u}^{2} t_{M} A_{u}^{V}-m_{d}^{2} t_{M} A_{d}^{V}
$$

Here, $u, d$ represent $t, b ; u_{M}^{i}, d_{M}^{i} ; \nu_{R}^{j}, l_{M}^{j}$. Two intermediate functions $A_{u}^{V}, A_{d}^{V}$ are expressed in terms of loop functions, $C, F[44]$ : 
- $H_{3}^{0} \rightarrow W^{+} W^{-}$

$$
\begin{aligned}
& A_{u}^{W}=\frac{1}{2}\left[C\left(m_{W}^{2} ; m_{u}^{2}, m_{d}^{2}\right)+F\left(m_{W}^{2} ; m_{u}^{2}, m_{d}^{2}\right)\right] \\
& A_{d}^{W}=\frac{1}{2}\left[C\left(m_{W}^{2} ; m_{d}^{2}, m_{u}^{2}\right)+F\left(m_{W}^{2} ; m_{d}^{2}, m_{u}^{2}\right)\right]
\end{aligned}
$$

- $H_{3}^{0} \rightarrow Z Z$

$$
\begin{aligned}
A_{f}^{Z} & =\left[\frac{\left(T_{3}-Q \sin ^{2} \theta_{W}\right)^{2}}{\cos ^{2} \theta_{W}}+\left(\frac{Q \sin ^{2} \theta_{W}}{\cos \theta_{W}}\right)^{2}\right] C\left(m_{Z}^{2} ; m_{f}^{2}\right) \\
& +\left[\frac{\left(T_{3}-Q \sin ^{2} \theta_{W}\right)^{2}}{\cos ^{2} \theta_{W}}-\left(\frac{Q \sin ^{2} \theta_{W}}{\cos \theta_{W}}\right)^{2}\right] F\left(m_{Z}^{2} ; m_{f}^{2}\right)
\end{aligned}
$$

$C, F$ are generally defined in terms of the 't Hooft-Veltman scalar loop integrals [44]. However, in this case we have top quark and heavy mirror fermions, which allows us to use asymptotic forms in the high-mass limit:

$$
\begin{gathered}
C\left(m_{V}^{2} ; m_{u}^{2}, m_{d}^{2}\right)=\int_{0}^{1} d x \int_{0}^{x} d y \frac{1}{D}, \\
F\left(m_{V}^{2} ; m_{u}^{2}, m_{d}^{2}\right)=-\int_{0}^{1} d x \int_{0}^{x} d y \frac{1}{D},
\end{gathered}
$$

where

$$
\begin{gathered}
D=m_{H_{3}^{0}}^{2}(1-x)(1-y)+m_{V}^{2}(1-x) \\
+m_{V}^{2} y(x-y)-m_{u}^{2}(1-y)-m_{d}^{2} y .
\end{gathered}
$$




\section{BIBLIOGRAPHY}

[1] Y. Nambu, Phys. Rev. Lett. 4, 380 (1960). doi:10.1103/PhysRevLett.4.380.

Y. Nambu and G. Jona-Lasinio, Phys. Rev. 122, 345 (1961). doi:10.1103/PhysRev.122.345

[2] F. Englert and R. Brout, Phys. Rev. Lett. 13, 321 (1964). doi:10.1103/PhysRevLett.13.321.

P. W. Higgs, Phys. Lett. 12, 132 (1964). doi:10.1016/0031-9163(64)91136-9.

P. W. Higgs, Phys. Rev. Lett. 13, 508 (1964). doi:10.1103/PhysRevLett.13.508.

[3] B. T. Cleveland, T. Daily, R. Davis, Jr., J. R. Distel, K. Lande, C. K. Lee, P. S. Wildenhain and J. Ullman, Astrophys. J. 496, 505 (1998). doi:10.1086/305343.

J. N. Abdurashitov et al. [SAGE Collaboration], Phys. Rev. C 60, 055801 (1999) doi:10.1103/PhysRevC.60.055801 [astro-ph/9907113].

W. Hampel et al. [GALLEX Collaboration], Phys. Lett. B 447, 127 (1999). doi:10.1016/S0370-2693(98)01579-2

Q. R. Ahmad et al. [SNO Collaboration], Phys. Rev. Lett. 87, 071301 (2001) doi:10.1103/PhysRevLett.87.071301 [nucl-ex/0106015].

K. Eguchi et al. [KamLAND Collaboration], Phys. Rev. Lett. 90, 021802 (2003) doi:10.1103/PhysRevLett.90.021802 [hep-ex/0212021].

[4] M. C. Gonzalez-Garcia and M. Maltoni, Phys. Rept. 460, 1 (2008) doi:10.1016/j.physrep.2007.12.004 [arXiv:0704.1800 [hep-ph]].

[5] K. A. Olive et al. [Particle Data Group Collaboration], Chin. Phys. C 38, 090001 (2014). doi:10.1088/1674-1137/38/9/090001

[6] P. A. R. Ade et al. [Planck Collaboration], arXiv:1502.01589 [astro-ph.CO].

[7] G. B. Gelmini and M. Roncadelli, Phys. Lett. B 99, 411 (1981). doi:10.1016/0370-2693(81)90559-1.

G. Gelmini and E. Roulet, Rept. Prog. Phys. 58, 1207 (1995) doi:10.1088/0034-4885/58/10/002 [hep-ph/9412278].

[8] P. Minkowski, Phys. Lett. B 67, 421 (1977); M. Gell-Mann, P. Ramond and R. Slansky, in Supergravity, eds. P. van Niewenhuizen and D. Z. Freedman (North Holland 1979); T. Yanagida, in Proceeding of Workshop on Unified 
Theory and Baryon Number in the Universe, eds. O. Sawada and A. Sugamoto (KEK 1979); S. L. Glashow, The future of elementary particle physics, in Proceedings of the 1979 Cargese Summer Institute on quarks and leptons (M. Levy, J. -L. Basdevant, D. Speiser, J. Speiser, R. Gatsmans, and M. Jacob, eds.) Plenum Press, New York, 1980, p. 687; R. N. Mohapatra and G. Senjanovíc, Phys. Rev. Lett. 44, 912 (1980); J. Schechter and J. W. F. Valle, Phys. Rev. D 22, 2227 (1980). For recent reviews, see V. Barger, D. Marfatia, and K. Whisnant, Int, J,. Mod. Phys. E12, 569 (2003) [arXiv:hepph/0308123]; R. N. Mohapatra et al, arXiv:hep-ph/0510213; G. Altarelli, arXiv:hep-ph/0611117, and references therein.

[9] J.C. Pati, A. Salam, Phys. Rev. D 10 (1974) 275; R. N. Mohapatra, J.C. Pati, Phys. Rev. D 11 (1975) 2558; G. Senjanović, R. N. Mohapatra, Phys. Rev. D 12 (1975) 1502; G. Senjanović, Nucl. Phys. B 153 (1979) 334.

[10] W.-Y. Keung, G. Senjanović, Phys. Rev. Lett. 50 (1983) 1427. A. Ferrari et al., Phys. Rev. D 62 (2000) 013001;

[11] V. Khachatryan et al. [CMS Collaboration], Eur. Phys. J. C 74, no. 11, 3149 (2014) [arXiv:1407.3683 [hep-ex]].

[12] T. D. Lee and C. N. Yang, Phys. Rev. 104, 254 (1956). doi:10.1103/PhysRev.104.254

[13] I. Montvay, DESY-87-147; Phys. Lett. B 199, 89 (1987); DESY-87-077.

[14] P. Q. Hung, Phys. Lett. B 649, 275 (2007) [hep-ph/0612004].

[15] P. Q. Hung, T. Le, V. Q. Tran and T. C. Yuan, JHEP 1512, 169 (2015) doi:10.1007/JHEP12(2015)169 [arXiv:1508.07016 [hep-ph]].

[16] J. F. Gunion, H. E. Haber, G. L. Kane and S. Dawson, Front. Phys. 80, 1 (2000).

[17] M. S. Chanowitz and M. Golden, Phys. Lett. B 165, 105 (1985).

[18] H. Georgi and M. Machacek, Nucl. Phys. B 262, 463 (1985).

[19] R. Godbole, B. Mukhopadhyaya and M. Nowakowski, Phys. Lett. B 352, 388 (1995) [hep-ph/9411324].

[20] A. Aranda, J. Hernandez-Sanchez and P. Q. Hung, JHEP 0811, 092 (2008) [arXiv:0809.2791 [hep-ph]]. 
[21] P. Q. Hung and P. Mosconi, hep-ph/0611001.

[22] M. E. Peskin and T. Takeuchi, Phys. Rev. D 46, 381 (1992).

[23] J. Beringer et al. [Particle Data Group Collaboration], Phys. Rev. D 86, 010001 (2012). We would like to thank Tim Tait for an earlier update.

[24] P. Q. Hung, Nucl. Phys. B 805, $326 \quad$ (2008) doi:10.1016/j.nuclphysb.2008.07.024 [arXiv:0805.3486 [hep-ph]].

[25] V. Hoang, P. Q. Hung and A. S. Kamat, Nucl. Phys. B 877, 190 (2013) doi:10.1016/j.nuclphysb.2013.10.002 [arXiv:1303.0428 [hep-ph]].

[26] S. Chatrchyan et al. [CMS Collaboration], Phys. Lett. B 716, 30 (2012) [arXiv:1207.7235 [hep-ex]]; G. Aad et al. [ATLAS Collaboration], Phys. Lett. B 716, 1 (2012) [arXiv:1207.7214 [hep-ex]].

[27] CMS Collaboration [CMS Collaboration], CMS-PAS-HIG-15-002.

[28] S. Chatrchyan et al. [CMS Collaboration], Phys. Rev. Lett. 110, 081803 (2013) [arXiv:1212.6639 [hep-ex]].

[29] V. Hoang, P. Q. Hung and A. S. Kamat, Nucl. Phys. B 896 (2015) 611-656, [arXiv:1412.0343 [hep-ph]].

[30] S. Chatrchyan et al. [CMS Collaboration], JHEP 1401, 096 (2014) [arXiv:1312.1129 [hep-ex]].

[31] S. Chatrchyan et al. [CMS Collaboration], arXiv:1312.5353 [hep-ex].

[32] S. Chatrchyan et al. [CMS Collaboration], Phys. Rev. D 89, 012003 (2014) [arXiv:1310.3687 [hep-ex]].

[33] S. Chatrchyan et al. [CMS Collaboration], arXiv:1401.5041 [hep-ex].

[34] S. Heinemeyer et al. [LHC Higgs Cross Section Working Group Collaboration], arXiv:1307.1347 [hep-ph].

[35] CMS Collaboration [CMS Collaboration], CMS-PAS-EXO-16-018.

[36] The ATLAS collaboration, ATLAS-CONF-2015-081.

[37] The ATLAS collaboration, ATLAS-CONF-2013-067, ATLAS-COM-CONF2013-082.

S. Chatrchyan et al. [CMS Collaboration], Eur. Phys. J. C 73, 2469 (2013) [arXiv:1304.0213 [hep-ex]]. 
[38] M. Spira, A. Djouadi, D. Graudenz and P. M. Zerwas, Nucl. Phys. B 453, 17 (1995) [hep-ph/9504378].

[39] G. Aad et al. [ ATLAS Collaboration], [arXiv:1409.6064 [hep-ex]].

[40] V. Khachatryan et al. [CMS Collaboration], [arXiv:1408.3316 [hep-ex]].

[41] J. F. Gunion, H. E. Haber and C. Kao, Phys. Rev. D 46, 2907 (1992).

[42] The ATLAS collaboration, ATLAS-CONF-2013-067, ATLAS-COM-CONF2013-082.

[43] W. Hollik, In *Langacker, P. (ed.): Precision tests of the standard electroweak model* 37-116, and Muenchen MPI Phys. - MPI-Ph-93-021 (93/04,rec.Sep.) 79 p. Bielefeld U. - BI-TP-93-16 (93/04,rec.Sep.) 79 p

[44] G. 't Hooft and M. J. G. Veltman, Nucl. Phys. B 153, 365 (1979). G. Passarino and M. J. G. Veltman, Nucl. Phys. B 160 (1979) 151.

[45] M. E. Peskin and D. V. Schroeder, Reading, USA: Addison-Wesley (1995) 842 p [ISBN-13:9780201503975]

[46] H. E. Haber and D. O’Neil, Phys. Rev. D 83, 055017 (2011) [arXiv:1011.6188 [hep-ph]].

[47] H. K. Dreiner, H. E. Haber and S. P. Martin, Phys. Rept. 494, 1 (2010) [arXiv:0812.1594 [hep-ph]]. 